\title{
Vestibular Rehabilitation for Dizziness and Balance Disorders after Concussion
}

\author{
by \\ Bara Abdel Majid Alsalaheen \\ Bachelor of Science in Physiotherapy, University of Jordan, 2006 \\ Masters of Science, University of Pittsburgh, 2008
}

Submitted to the Graduate Faculty of School of Health and Rehabilitation Sciences in partial fulfillment

of the requirements for the degree of

Doctor of Philosophy in Rehabilitation Sciences

University of Pittsburgh 


\title{
UNIVERSITY OF PITTSBURGH
}

\section{School of Health and Rehabilitation Sciences}

This dissertation was presented

by

\author{
Bara A. Alsalaheen \\ It was defended on
}

Oct, 30th, 2012

and approved by

Anthony P. Kontos, PhD, Department of Orthopedic Surgery, University of Pittsburgh

Joseph M. Furman MD, PhD, Professor, Department of Otolaryngology, University of Pittsburgh

Gregory F. Marchetti PT, PhD, Associate Professor, Department of Physical Therapy, Duquesne University

Susan L. Whitney PT, PhD, Professor, Department of Physical Therapy, University of Pittsburgh

Thesis Director/Committee Chair

Patrick J. Sparto, PT, PhD, Associate Professor, Department of Physical Therapy, University of Pittsburgh 
Copyright (C) by Bara A. Alsalaheen

2012 


\title{
Vestibular Rehabilitation for Dizziness and Balance Disorders after Concussion
}

\author{
Bara Alsalaheen, $\mathrm{PhD}$ \\ University of Pittsburgh, 2012
}

Many individuals who acquire a concussion are seen for vestibular rehabilitation. The studies describing the prescribed exercises and the outcomes of vestibular rehabilitation are sparse.

Additionally, the outcome measures that have been used lack normative reference values for healthy adolescents and have not been examined for correlation with established neurocognitive measures. Therefore, the purpose of this dissertation is to provide normative data for the measures used in vestibular rehabilitation, to describe the exercises and the outcomes of a vestibular physical therapy program and to examine the relationship between self-reported symptoms, neurocognitive and balance performance in individuals referred to vestibular physical therapy.

A cross - sectional design was used to establish normative reference values; Ninety- one participants completed the Activities - specific Balance Confidence scale, Dynamic Gait Index, Functional Gait Assessment, Timed "UP \& GO”, Five Times Sit to Stand test, tests of gait speed and the Balance Error Scoring System. Percentile scores were computed for all measures.

A retrospective chart review of 114 consecutive subjects referred for vestibular physical therapy after concussion was performed. At the time of initial evaluation and discharge, recordings were made of outcome measures of self-report and balance performance. A repeatedmeasures mixed ANOVA tested whether there was an effect of time and age on the outcome measures. Frequency counts of the most common exercise types were recorded. A correlation 
analysis was performed to examine the relationship between balance measures and Immediate Post-concussion Assessment and Cognitive Testing (ImPACT) at the initiation of vestibular physical therapy and to examine the relationship between change in ImPACT and change in balance measures.

Normative reference values for balance were provided. An improvement was observed in all measures at the time of discharge from vestibular rehabilitation. Eye-Head Coordination exercises were the most commonly prescribed exercise type. Significant relationships between the ImPACT neurocognitive scores and balance measures at the start of vestibular rehabilitation were supported.

In conclusion, Individuals who received vestibular physical therapy after concussion had favorable outcomes, but it is not possible to determine if the outcomes were solely due to the therapy. The interventions provided by physical therapists were consistent across patients. 
TABLE OF CONTENTS

1.0

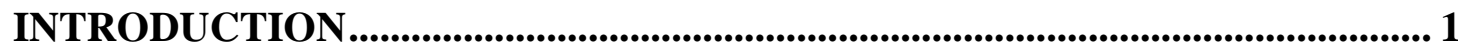

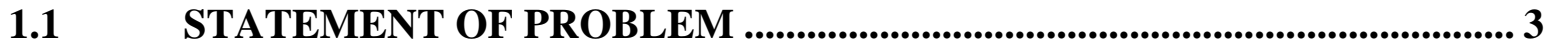

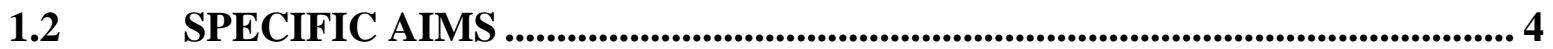

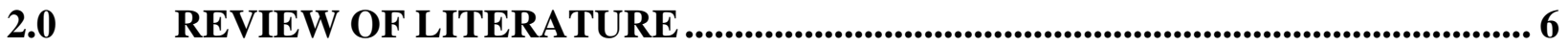

2.1 DEFINITION AND GRADING OF MILD TRAUMATIC BRAIN INJURY

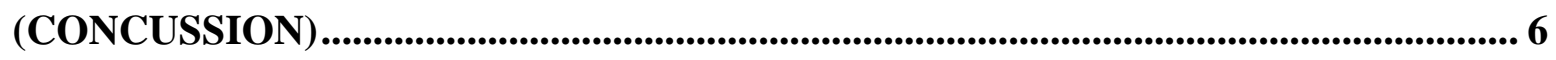

2.2 PREVALENCE OF MILD TRAUMATIC BRAIN INJURY ........................9

2.3 EFFECTS OF CONCUSSION ON NEUROCOGNITIVE PERORMANCE,

BALANCE AND POSTURE, AND SELF- REPORT SYMPTOMS ......................... 10

2.3.1 Neurocognitive performance............................................................... 10

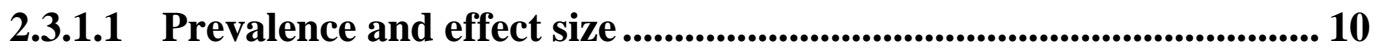

2.3.1.2 Instruments for neurocognitive testing after concussion ................. 12

2.3.2 Balance and posture............................................................................ 17

2.3.3 Self- report symptoms...................................................................... 19

2.4 MULTIFACATED RECOVERY PROCESS AFTER CONCUSSION....... 25

2.4.1 Predictors of recovery after concussion ................................................. 25

2.4.1.1 Initial markers of concussion severity .......................................... 25

2.4.1.2 Age 


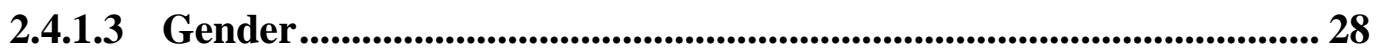

2.4.1.4 Concussion History ...................................................................... 29

2.4.2 Correlation between outcome measures among different domains during recovery30

$\begin{array}{llllll}2.5 & \text { VESTIBULAR } & \text { REHABILITATION } & \text { FOR } & \text { PATIENTS } & \text { POST }\end{array}$

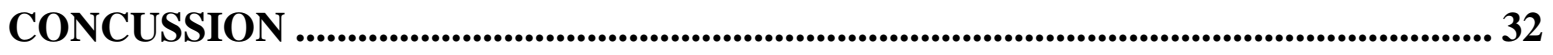

2.5.1 Evidence behind vestibular rehabilitation for concussion .......................... 32

2.5.2 Outcome measures used in vestibular rehabilitation .................................. 34

2.5.3 Limitations of previous work and rationale for proposed studies .......... 34

2.5.3.1 Rationale for the first study ...................................................................... 35

2.5.3.2 Rationale for the second study .............................................................. 35

2.5.3.3 Rationale for the third study .................................................................... 36

2.5.3.4 Rationale for the fourth study.................................................................. 37

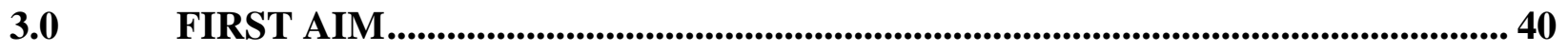

INTRODUCTION ............................................................................................... 40

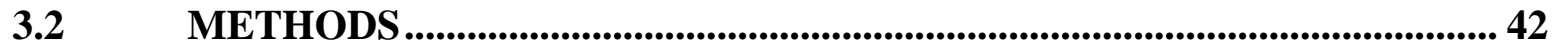

3.2.1 Design ...................................................................................................................... 42

3.2.2 Subjects: ............................................................................................................... 43

3.2.3 Outcome measures .................................................................................................. 44

3.2.4 Procedure.................................................................................................................. 47

3.2.5 Statistical analysis:............................................................................................. 48

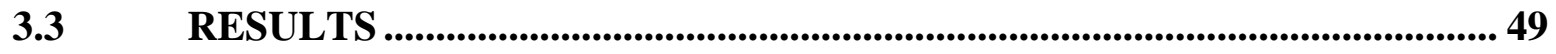

3.3.1 Reference values............................................................................................ 50 


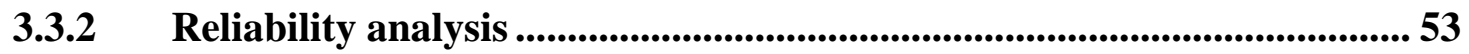

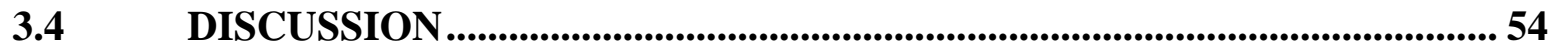

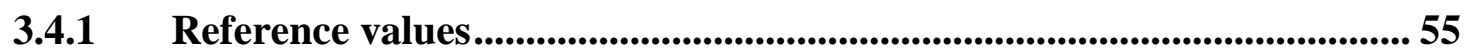

3.4.2 Reliability analysis:......................................................................................... 58

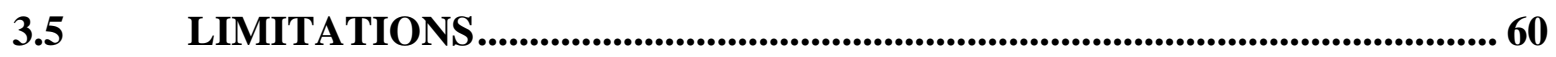

CONCLUSION ...................................................................................................... 60

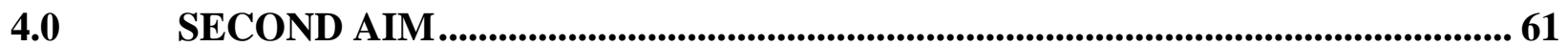

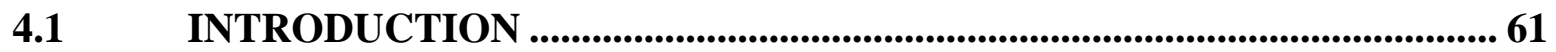

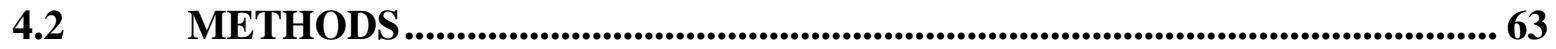

4.2.1 Participants.......................................................................................................... 63

4.2.2 Intervention and outcome measures ............................................................. 63

4.2.2.1 Self-report measures ................................................................................... 64

4.2.2.2 Gait and balance Performance measures ............................................. 65

4.2.3 Statistical analysis ........................................................................................ 66

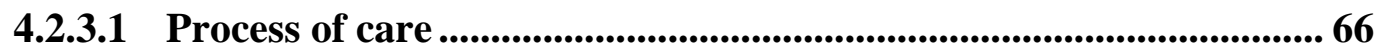

4.2.3.2 Outcome measures .................................................................................... 66

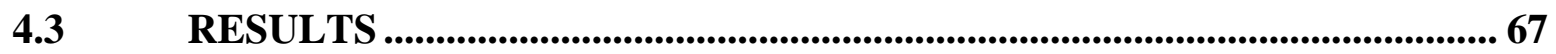

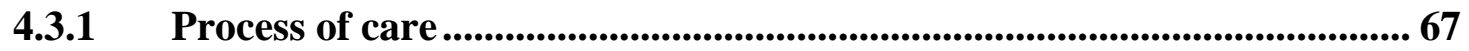

4.3.2 Outcome measures at initial evaluation ..........................................................68

4.3.3 Change in outcome measures....................................................................... 69

D.4 DISCUSSION ..................................................................................................... 72

$4.5 \quad$ LIMITATIONS AND FUTURE DIRECTIONS .............................................. 76

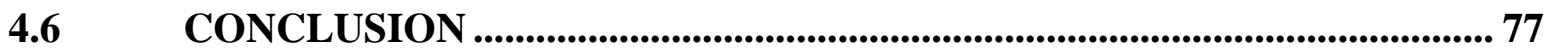




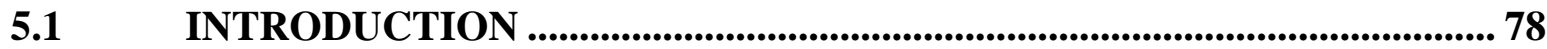

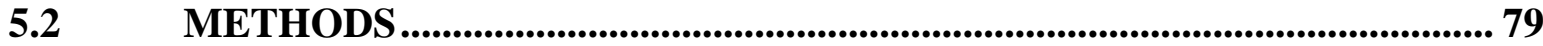

5.2.1 Participants......................................................................................................... 79

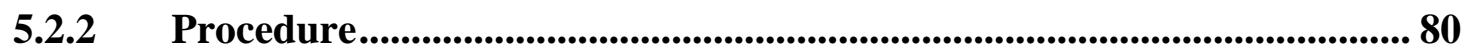

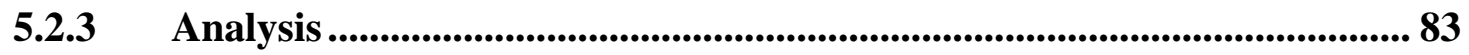

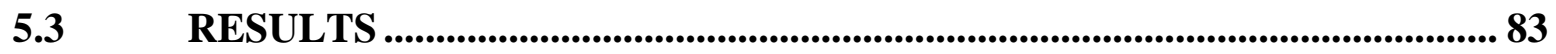

5.3.1 Preferred Prescription Patterns ...................................................................... 86

5.3.2 Progression .......................................................................................................... 87

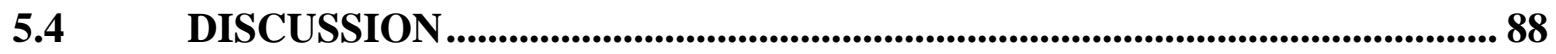

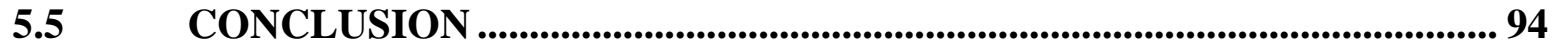

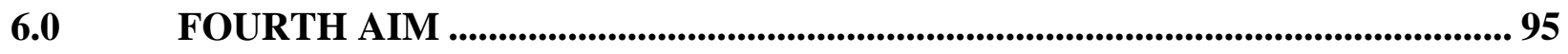

6.1 INTRODUCTION .............................................................................................. 95

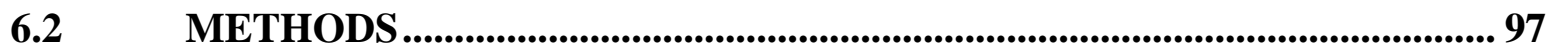

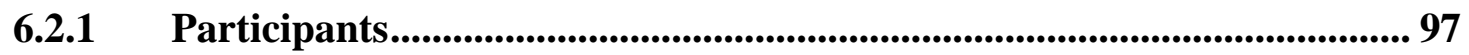

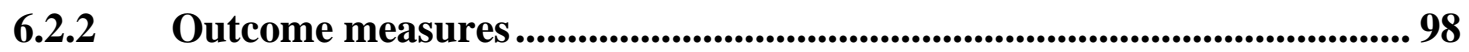

6.2.2.1 Self report balance measures ...............................................................98

6.2.2.2 Balance performance measures ............................................................. 99

6.2.2.3 Neurocognitive and symptom measures .............................................. 100

6.2.3 Statistical analysis ......................................................................................... 101

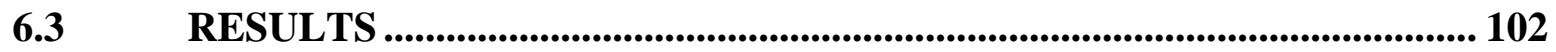

6.3.1 Analysis at rehab start...................................................................................... 102 
6.3.1.1 Relationship between clinical balance and neurocogntive measures 103

6.3.2 Analysis of change scores ……......................................................................... 105

6.4 DISCUSSION ...................................................................................................... 109

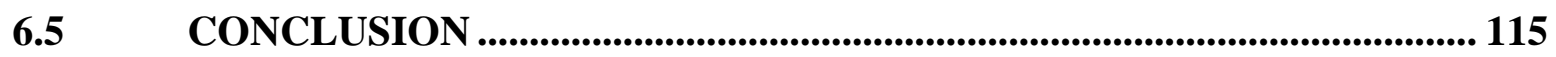

7.0 FUTURE CONSIDERATIONS AND FUTURE DIRECTIONS ......................... 116

7.1 FUTURE CONSIDERATIONS ...................................................................... 116

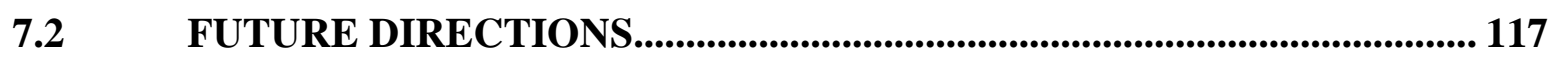

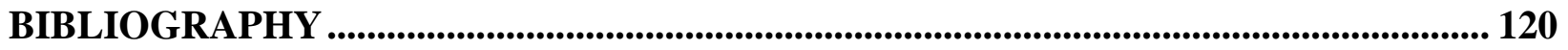




\section{LIST OF TABLES}

Table 2-1: The ImPACT Neuropsychological Testing Battery ............................................. 16

Table 2-2: Clusters of self- report symptoms after concussion ........................................ 19

Table 2-3: Total symptoms score reported after concussion ............................................... 21

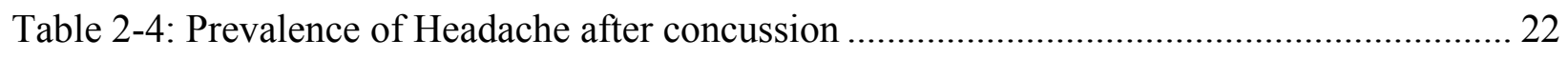

Table 2-5: Prevalence of Dizziness after concussion ...................................................... 24

Table 2-6: Prevalence of Balance problems after concussion ........................................... 24

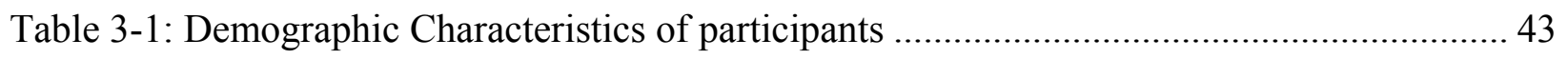

Table 3-2: Number of participants who participated in formal athletic activities ..................... 44

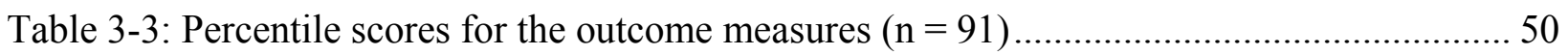

Table 3-4: Percentile scores for individual items on the Activities-specific Balance Confidence

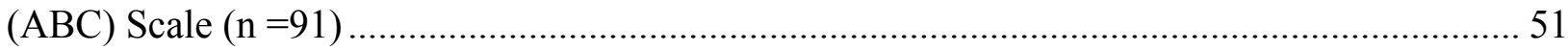

Table 3-5: Percentile scores for individual items on the Functional Gait Assessment (FGA).... 52

Table 3-6: Percentile scores for the Balance Error Scoring system (BESS). L ower scores

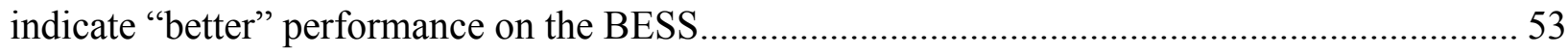

Table 3-7: Reliability coefficients, standard error of the measurement (SEM), and minimal

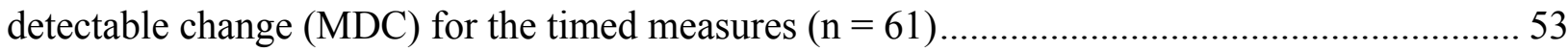


Table 3-8: Percent agreement, expected agreement, and weighted kappa statistics for the individual conditions of the Balance Error Scoring System (BESS). 54

Table 4-1: Mean (SD) of demographic and outcome measures at time of initial evaluation for vestibular rehabilitation, according to those participants who had evaluation only, and those who returned for a least 1 additional visit 70

Table 4-2: Mean (SD) of outcome measures at times of initial evaluation and discharge 71 Table 4-3: Mean (SD) for the significant interaction effect between age group and time on dizziness severity and Sensory Organization Test (SOT) scores. 72

Table 5-1: The exercise modifiers used the vestibular rehabilitation exercises 82 Table 5-2: Summary of the most common prescribed exercises throughout therapy and during the first visit in number of subjects (Percentage of subjects) 85 Table 5-3: Progression patterns for the VORx1 exercise $\uparrow$ 88 Table 6-1: Relationship between ImPACT composite scores and measures administered at the start of vestibular physical therapy 104 Table 6-2: The relationship between outcome measures administered at the start of vestibular rehabilitation, total symptom score and "vestibular/ balance" subset of symptoms..... 105 Table 6-3: Difference in the initial outcome measures between subjects not included in change analysis $(n=54)$ and subjects included in change analysis $(n=39)$ 107 Table 6-4: Relationship between change in Vestibular/ balance measures and change in imPACT composites and the total symptom score 108 Table 6-5: Relationship between change in symptom scores and change in measures administered over the course of vestibular rehabilitation 109 


\section{PREFACE}

Over the past five years I have received support and encouragement from a great number of individuals. Without their valuable insights and help, this dissertation would have not moved from an idea to a completed study. I would like to express my deepest appreciation to my committee chair, Patrick Sparto, PhD, PT, for the patient guidance, encouragement and advice he has provided throughout my time as his student. His guidance has made this a thoughtful and rewarding journey. I would also like thank Susan Whitney, PhD, PT, NCS, ATC, FAPTA, for her continuous assistance, insights, and support she provided throughout the time when I was a graduate student at the University of Pittsburgh.

I would like to acknowledge the members of my committee including Joseph Furman, $\mathrm{MD}, \mathrm{PhD}$, Anthony Kontos $\mathrm{PhD}$, Gregory F. Marchetti $\mathrm{PT}, \mathrm{PhD}$ for their help in the conceptualization and refining the specific aims of this dissertation and for the methodological insights they have provided. I have been extremely lucky to have members of my doctoral committee who cared so much about my work, and who responded to my questions and queries so promptly.

I would like to thank Micky Collins PhD, Mark Lovell PhD, Tanya Hagen, MD, Jamie Pardini PhD, and Vanessa Fazio, PhD of the UPMC Sports Medicine Concussion program. In addition, I would like to thank the physical therapists of the Centers for Rehab Services Vestibular Rehabilitation Clinic: Anne Mucha, DPT, NCS, Laura Moris, PT, NCS, Corrie 
Amick, DPT, Kathryn Brown, MS, PT, NCS, Dana Hinderliter, PT, and Darcy Nunn, DPT, NCS.

I would like to thank the graduate students from the University of Pittsburgh and the physical therapists from the Centers for Rehab Services of University of Pittsburgh Medical Center for assisting in the data collection, and students from Quaker Valley, Oakland Catholic and Central Catholic High Schools for participating in the first aim of this dissertation.

I would like to thank the Clinical and Translational Science Institute (CTSI) and Chuck Vukotich at the University of Pittsburgh for help in recruiting the schools, and the Department of Physical Therapy in the University of Pittsburgh for providing the equipment for testing.

I must express my thankfulness for my parents and siblings for their unconditional support and continuous belief in me since I was young. They all have experienced the ups and downs of my academic journey.

I would like to express my deepest love and gratitude to Mariam, my fiancé; if I wrote down everything I ever wanted in a fiancé and a best friend I would not have believed I could meet someone better! All of your continuous support after moving to Michigan is deeply appreciated. Thanks for making me finish this thing! I was continually amazed by yo ur willingness to proofread countless pages and repeatedly listen to my dissertation presentation. 


\subsection{INTRODUCTION}

Traumatic brain injury (TBI) is one of the most prevalent acquired neurological conditions occurring in children and young adults. ${ }^{1-2}$ Most TBI is classified as mild TBI (i.e. concussion). ${ }^{3-4}$

The annual rate of mTBI is $130-546$ per 100,000 persons. $^{5-7}$ The reported numbers of concussion are suggested to be conservative numbers; sport-related concussions are estimated to be 1.6 to 3.8 million annually. ${ }^{8}$ The deleterious effect of concussion appear in the areas of neurocognitive functioning, ${ }^{9-12}$ postural and balance control, ${ }^{13-16}$ and self-report symptoms. ${ }^{17-21}$

Neurocognitive evaluation is seen as the cornerstone of concussion assessment and is the area where the most advances in research have been made in the management of concussion. ${ }^{22-24}$

Computerized neurocognitive assessment (e.g.; Immediate Post-Concussion Assessment and Cognitive Testing, CogSport) has replaced the traditional paper and pencil assessment, and has provided a means to track the recovery process. ${ }^{12}$ One of the most studied computerized tools is the Immediate Post-Concussion Assessment and Cognitive Testing (ImPACT) ${ }^{24}$

ImPACT contains a symptom inventory and neurocognitive test battery that examines the neurocognitive aspects of verbal memory, visual memory, reaction time and processing speed.

Dizziness and balance difficulties have been widely reported after concussion. ${ }^{25-29}$ While the dizziness and balance impairments usually resolve in the first 3-5 days after concussion, ${ }^{14,30-}$ ${ }^{31}$ some patients exhibit persistent dizziness and balance disorders that require rehabilitation. ${ }^{32-40}$ 
While the vestibular origin of dizziness and postural instability after concussion has been reported in many studies, ${ }^{34,41-43}$ few studies have investigated the effect of vestibular rehabilitation on patients with post concussion symptoms. ${ }^{34,39,44}$

Over the course of vestibular rehabilitation, various gait and functional balance measures are used, and many of the clinical decisions regarding the exercise initiation / progression or discharge are based on the scores obtained through the gait and balance testing as w ell as symptom resolution. However, most of these measures were initially developed to be used in adults (mostly older adults) and yet no study has investigated the reliability and the normative scores of these measures in children between the age of 13 and 18. By providing age -specific normative scores, we will be able to use these normative scores to determine the impact of concussion on children's balance performance and to provide an end point for vestibular rehabilitation therapy.

The results of vestibular rehabilitation after concussion revealed that vestibular rehabilitation exercises may reduce dizziness and balance disorders. ${ }^{34,44}$ However, these studies did not describe the severity of self-reported dizziness, and did not describe the severity of balance performance dysfunction in the patients seen for vestibular rehabilitation after concussion. Furthermore, it is unknown if the amount of recovery made during vestibular rehabilitation is different between children and adults.

Although the individualized nature of vestibular rehabilitation programs for individuals after concussion has been emphasized by experts vestibular physical therapists, ${ }^{45}$ an understanding of what exercises have been prescribed for this population may be useful for several reasons. First, due to the customized nature of vestibular rehabilitation exercises, the exercise prescription can provide a detailed picture of the specific impairments and functional 
limitations encountered by the individuals with concussion. Second, it can serve as a foundation for other therapists who may be starting vestibular rehabilitation programs for management of individuals with concussion. Third, by a nalyzing the prescription patterns of experienced therapists, we may begin to examine how exercise progression relates to outcomes.

The impairments of neurocognitive and balance function after concussion are well documented in the literature. ${ }^{9,12,19,24,30-31,46-50}$ However, they have been studied individually. ${ }^{48}$

There are a limited number of studies that relate the neurocognitive testing scores to functional gait and balance measures post-concussion. ${ }^{21}$ Examining the relationship between neurocoginitive testing and functional balance measures utilized in clinical settings may help clinicians effectively use these easily accessed measures in clinical decision making, and may provide us with a better understanding of the multi faceted nature of the recovery process after concussion.

In conclusion, many limitations have surrounded the implementation of vestibular rehabilitation in the management of patients post- concussion. By addressing these limitations, vestibular rehabilitation will have a greater impact in the management of patients with concussion.

\subsection{STATEMENT OF PROBLEM}

Studies of vestibular rehabilitation for dizziness and balance disorders after concussion have been promising. However, for individuals between 13 and 18 of age treated with vestibular rehabilitation, there is a 1 ack of normative reference values for the common clinical balance 
outcome measures used to track recovery. The severity of dizziness and imbalance problems in this group of individuals post - concussion has not been described and the change in gait and balance outcomes over the course of vestibular rehabilitation has not been quantified in children and adult patients. Moreover, the specific exercise prescriptions during the course of vestibular rehabilitation have not been detailed. Neurocognitive and balance performance and recovery after concussion have been studied separately, and there have been no studies to examine the presence/absence of relationship between neurocognitive and balance recovery. There have been no studies to examine if the markers of concussion severity relate to vestibular rehabilitation outcomes.

\subsection{SPECIFIC AIMS}

1. A cross - sectional study was conducted to provide normative data and to examine the reliability for clinical gait/ balance measures for healthy high school aged children.

2. A retrospective chart review was conducted: 1) to describe the severity of dizziness and balance dysfunction in patients who were referred to vestibular rehabilitation after being diagnosed with concussion, 2) to describe the outcomes of vestibular rehabilitation for dizziness and balance dysfunction after concussion, and 3) to examine whether the amount of recovery made over the course of vestibular rehabilitation is equal between children and adults.

3. A retrospective case series was performed to describe exercise prescription patterns in patients treated with vestibular rehabilitation after concussion. The analysis will describe 
the progression of the exercises and common exercise volumes for vestibular rehabilitation exercises used in the management of individuals with dizziness and balance disorders after concussion.

4. A retrospective case series was performed to examine the relationship between neurocognitive and balance performance and recovery after concussion. 


\subsection{REVIEW OF LITERATURE}

\subsection{DEFINITION AND GRADING OF MILD TRAUMATIC BRAIN INJURY (CONCUSSION)}

Over the last three decades, many overlapping and sometimes confusing terms and constructs were used to describe the milder spectrum of head injuries $;{ }^{51}$ minor head injury, mild closed head injury, mild traumatic brain injury, and concussion were seen throughout the literature. Although there were slight differences between these terms, the differences are often overlooked when interpreting the evidence related to prevalence and recovery.

Many professional organizations have adopted the use of one of the above mentioned terms; the World Health Organization (WHO) and the American Congress of Rehabilitation Medicine have used mild traumatic brain injury (mTBI). ${ }^{52-53}$ Concussion and sports- concussion were used by the American Academy of Neurology and Concussion in Sport group,

respectively. $^{22,54}$ The American Academy of Pediatrics has used the term minor closed head injury. $^{55}$

Loss of consciousness, amnesia, and confusion are considered the hallmarks of concussion, and were often included as defining characteristics in the various definitions of concussion. However, there was high variability in numerical value assigned to some of these defining characteristics; for example the duration of loss of consciousness (LOC) at time of 
concussion has ranged between 0-30 minutes, and the duration of post-traumatic amnesia (PTA) has ranged between 0-24 hours. ${ }^{49}$ The variability in the selected term of use and the duration of defining characteristics has led to great heterogeneity in the selected "concussion" populations across the studies, and sparked a great amount of confusion over the years.

Over the last three decades many definitions have been proposed for concussion (i.e. $\mathrm{mTBI}$ ). One of the earliest and most widely recognized definitions of concussion was proposed by the committee on head injury nomenclature of neurologic surgeons in $1966 \mathrm{in}$ which concussion was defined as "a c linical syndrome characterized by the immediate and transient posttraumatic impairment of neural function such as alteration of consciousness, disturbance of vision or equilibrium, etc., due to brain stem dysfunction" ${ }^{56}$ In 1997, the American Academy of Neurology (AAN) defined concussion as "Any trauma induced alteration in mental status that may or may not include loss of consciousness".54

Recently the Centers for Disease Control (CDC) have put forth the following definition for concussion: "a complex pathophysiologic process affecting the brain, induced by traumatic biomechanical forces secondary to direct or indirect forces to the head. It is caused by a blow or jolt to the head that disrupts the function of the brain. This disturbance of brain function is typically associated with normal structural neuroimaging findings (i.e., CT scan, MRI). It results in a constellation of physical, cognitive, emotional and/or sleep-related symptoms and may or may not involve LOC. ${ }^{2}$ The CDC has also stated the mTBI and concussion are synonymous and are often used interchangeably. ${ }^{2}$

Although the various definitions of concussion were not intended to provide subtypes for concussion severity, many have detailed the defining characteristics in order to establish grading scales for concussion severity. The establishment of different grading scales, variability in the 
numerical values assigned to the defining characteristics, and the use of these subscales as guidelines to the recovery process are all factors that have led to debate about the definition, prognosis and management of concussion over many years. ${ }^{57}$

There have been at least 17 grading scales for concussion in the literature ${ }^{57}$ It is essential to note that none of these scales was validated by prospective data or based on e mpiric evidence. ${ }^{58}$ Grading scales reflect a consensus among a group of experts and were based on clinical experience. ${ }^{57,59-60}$ The grading scales also heavily based the classification of severity on the presence and duration of LOC and other markers of severity. The grading scales have also assumed universal effects of concussion for all age and gender groups.

Recent evidence suggests no strong correlation between LOC and neurocognitive testing performance and recovery. ${ }^{61-62}$ Furthermore, some studies have suggested differential rates of recovery for different age groups ${ }^{63-66}$ and genders. ${ }^{67-69}$

The absence of a discernible relationship between LOC and neurocognitive recovery, and the presence of a differential rate of recovery for different age groups and gender invalidate the use of standardized concussion grading scales to make clinical decisions regarding return to play or work after concussion. ${ }^{57}$ The lack of a validated grading scale has led to the endorsement of an individualized concussion management approach. This approach was endorsed by the National Athletic Trainers Association in which neurocognitive performance, balance performance and resolution of self- report symptoms are used to track the recovery process. ${ }^{57,60,70-72}$ 


\subsection{PREVALENCE OF MILD TRAUMATIC BRAIN INJURY}

Traumatic brain injury (TBI) is one of the most prevalent acquired neurological conditions occurring in children and young adults. ${ }^{8}$ Epidemiological studies revealed that between $75 \%$ $90 \%$ of TBI are classified as mild TBI (i.e. concussion), ${ }^{73-74}$ the annual rate of mTBI is $130-546$ per 100,000 persons $^{5-7}$

Sports-related concussions have received the most attention; approximately 300,000 sports-related concussions occur in the United States every year. ${ }^{75}$ Three to eight percent of high school and college athletes sustain a concussion every year. ${ }^{76-78}$ Six percent of all sports injuries among the high school athletes are concussions, ${ }^{79}$ and $34 \%$ of college athletes have had at least one concussion in their past. ${ }^{80}$

The large number of reported concussions has led to concussion being recognized as one of the most common neurological injuries in contact sports. ${ }^{81}$ However, the rates of reported concussions vary by sport and age; American football accounts for $63 \%$ of high school concussions. $^{79}$

Despite the growing number of reported concussions in recent years, many researchers believe the actual numbers of concussions in sport and non-sport environments are much higher than reported; ${ }^{7,82-85}$ the estimations of concussions occurring in the U.S have ranged between 1 4 million concussions every year. ${ }^{8,86-87}$ The reported numbers of concussions are suggested to be conservative numbers because it is influenced by the variability of concussion definitions and therefore diagnosis. Additionally, athletes may not recognize that the symptoms they have is a result of a concussion, or may intentionally underreport the concussion symptoms due to personal desires, fear of financial loss or jeopardizing their future athletic career. ${ }^{83,88}$ One study reported $50 \%$ of interscholastic athletes do not report their concussion to medical personnel. ${ }^{83}$ 
Despite the uncertainty surrounding the actual number of concussions occurring in the United States every year, concussion is now being recognized as an epidemic and major public health concern, especially for children and young adults. ${ }^{23,54,79,89-90}$

The estimated annual cost (direct and indirect) of concussions in the United States ranges between $12-17$ billion dollars. ${ }^{73,91}$ Furthermore, concussion is found to have negative effect on psychological well being and health related quality of life (HRQOL), ${ }^{92-93}$ and also linked to higher family burden and emotional distress. ${ }^{94}$

\subsection{EFFECTS OF CONCUSSION ON NEUROCOGNITIVE PERORMANCE, BALANCE AND POSTURE, AND SELF- REPORT SYMPTOMS}

\subsubsection{Neurocognitive performance}

\subsubsection{Prevalence and effect size}

The effect of concussion on neurocognitive functioning has been documented in a large number of studies; ${ }^{49,76-77,80,95-102}$ neurocognitive testing is often seen as the cornerstone for concussion assessment and the domain that provides information about impairments and the recovery process after concussion. ${ }^{20,22-24,103-104}$ However, due to a practice effects, and continuous development in adolescents' cognitive abilities, return to baseline neurocognitive performance may not always indicate full recovery. ${ }^{23}$ Although neurocognitive testing is viewed as the cornerstone for concussion assessment, there is a consensus that other measures such as postural 
stability and self- report symptoms should be used in conjunction with neurocognitive testing. ${ }^{20}$ 57, 60, 70-71, 105-107

Despite the large body of evidence supporting the negative effect of concussion on neurocognitive testing, the extent to which the concussion affects cognitive abilities and the course of recovery has been controversial for decades. ${ }^{46}$ Many factors may contribute to the variability in the prevalence of neurocognitive effects of concussion and its course of recovery; studies had different operational definitions of concussion (if defined) and therefore the selection of patients was variable. ${ }^{46,108}$ For example, selection of subjects from the emergency department registry may under-represent the less severe spectrum of concussion patients and therefore inflate the effects of concussion. ${ }^{108-109}$ Conversely, selection of participants from sports settings where concussions are often not formally diagnosed by physicians and are often self- reported may under-represent the more severe continuum of individuals with concussion. ${ }^{9}$ Additionally, there are many different neurocognitive domains such as attention, executive functioning, fluency, delayed memory, memory acquisition, and visuospatial abilities. Within each domain, there are many instruments used to track the recovery of neurocognitive functioning. The heterogeneity of instruments and neurocognitive domains they include may explain some of the variability seen in the reported effect size of concussion across many studies. ${ }^{46}, 52$ The differences in the psychometric properties (e.g. sensitivity, specificity, and test-retest reliability) of different measures at different points throughout the course of recovery may also influence the reported effect size. ${ }^{110-111}$ Other methodological differences between studies (e.g. pre-injury selfcomparison vs. matched control) may affect the reported period of recovery., 46,112

Five meta analyses examined the effect of concussion on neuropsychologcal testing ${ }^{9,19,}$ 46, 113-114 The overall effect size ranges from small (e.g. $\mathrm{d}=.12),{ }^{114}$ to moderate $(\mathrm{d}=.54)^{46}$ and is 
comparable between the sports- concussion $(\mathrm{d}=.49),{ }^{9}$ and non-sport mTBI $(\mathrm{d}=.54) .{ }^{46}$ The overall effect size has been examined by the time since injury; Schretlen et al ${ }^{115}$ demonstrated a decrease in the effect size of mTBI on neurocognitive testing with time since concussion compared to matched controls; in this study, the effect sizes were calculated for four time periods since concussion; $<7$ days after mTBI, 2-29 days, 30- 89 days, and $>3$ months, and significant differences were found among effect sizes across these time intervals $(\mathrm{d}=.41, .29, .08,0.04$, respectively). ${ }^{115}$

Some studies examined the effect size for different domains of neurocognitive functioning rather than overall effect size; Zakzanis examined the effect of concussion on seven cognitive domains and found moderate to large effect sizes ranging from $(\mathrm{d}=.44)$ for manual dexterity to $(\mathrm{d}=.72)$ for flexibility/abstraction. ${ }^{113}$ In another meta analysis, most effect sizes of 9 cognitive domains were moderate to large. ${ }^{46}$ Belanger et al reported the largest acute effects of concussion for the domains of delayed memory, memory acquisition, and global cognitive functions ( $\mathrm{d}=1.00,1.03$, and 1.42 , respectively). ${ }^{9}$ Despite the decrement in total effect size since time of injury, ${ }^{9,19,46}$ various cognitive domains demonstrated great heterogeneity between the effect size, and therefore differential rate of recovery., ${ }^{9,46,114}$

In summary, concussion has negative effects on neurocognitive testing performance. The overall effect usually decreases with time after concussion. However, concussion may have greater or longer effects on certain aspects of neurocognitive functioning compared with others. ${ }^{9}$

\subsubsection{Instruments for neurocognitive testing after concussion}

Many instruments have been used to assess neurocognitive performance after concussion.

Traditionally, paper and pencil instruments were used. While paper and pencil measures have been somewhat effective in the diagnosis and management of concussion, paper and pencil 
instruments were not sensitive, specific, and did not have normative data. ${ }^{59,96}$ Moreover, paper and pencil testing is inconvenient and expensive to administer because it requires a trained professionals (e.g. a neuropsychologist) to administer and interpret the scores. ${ }^{57,59,106,116}$ Some of the paper and pencil tests (e.g. Symbol Digit Modalities Test (SDMT), Verbal Hopkins Learning Test (VHLT), and Trail Making Test (TMT) have also been criticized for a practice effect. $^{96,116-118}$

Studies that examined the recovery of neurocognitive deficits using different paper and pencil instruments revealed resolution of measurable neurocognitive deficits by the fifth day post concussion. $^{31,76,80,95-97,100,119}$ However, there are other studies that demonstrated persistent neurocognitive deficits in divided and sustained attention, ${ }^{120-121}$ processing speed, ${ }^{122}$ and reaction times ${ }^{120}$ long after the first five days post concussion.

In recent years, a shift has occurred with neurocognitive testing in which computerized

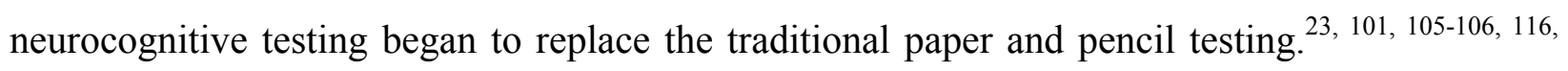
123-124 This trend in neurocognitive testing was endorsed by the first and second international symposium about concussion in sport ${ }^{22,103}$ and by the National Athletic Trainers Association. ${ }^{125}$

Computerized neurocognitive testing does not require a trained professional to administer, allowing more cost- and time-effective testing for large groups of individuals. ${ }^{57,110}$

The computerized software allows for increased accuracy in recording reaction times which may lead to better validity. The randomization of test stimuli may reduce practice effects of multiple administrations and may improve the reliability compared with "paper and pencil" neurocognitive evaluations. ${ }^{65,106-107,126-127}$

The implementation of computerized neurocognitive testing led to an increase in the number of "at risk individuals" with available baseline neurocognitive data, and opened an 
avenue for examining the recovery process after concussion on a larger scale. ${ }^{60,126}$ The recovery process after concussion has been examined by using matched controls or return to pre- injury baselines. ${ }^{128}$ The use of baseline testing is recommended whenever possible. ${ }^{22,57,70,103,128-129}$

Computerized neurocognitive testing demonstrated sensitivity to the initial neurocognitive deficits after concussion. ${ }^{65,123-124,130-132}$ Computerized neurocognitive testing is proven to be sensitive to the persistent neurocognitive deficits beyond the one week window of recovery seen in most patients with concussion. ${ }^{66,130}$

Many computerized neurocognitive testing assessment batteries are currently used in concussion assessment; CogSport, ${ }^{133}$ Headminder's Concussion Resolution Index (CRI), ${ }^{123}$ and the Immediate Post Concussion Assessment and Cognitive Testing (ImPACT) ${ }^{24}$ have been developed and used for neurocognitive testing.

CogSport was designed to measure changes in cognitive functioning in the areas of psychomotor, decision making, problem solving, and memory scales. ${ }^{134}$ CogSport was originally validated in Australian professional football players and healthy controls, ${ }^{126,131}$ and CogSport indices have demonstrated high to very high $(.69-.90)$ test re-test reliability. CogSport indices have been externally validated (i.e. construct validity) against the paper and pencil tests of Trail Making and Digit Symbol Substitution Tests. ${ }^{116}$

HeadMinder's Concussion Resolution Index (CRI) is an internet-based test that measures cognitive functioning in the areas of reaction time, processing speed, and visual recognition. It also includes a symptom scale. The CRI has been validated against a number of paper and pencil measures. ${ }^{123-124,135}$

ImPACT is a computer administered software package that containing a neurocognitive testing battery and a post concussion symptom scale. ${ }^{24}$ The neurocognitive testing of ImPACT 
measures different areas of cognitive functioning including attention, processing speed, reaction time, and memory. The ImPACT neurocognitive battery uses six test modules (word memory, design memory, X's and O's, symbol match, color match, and three letter memory) to generate four composite scores. Each testing module may contribute to more than one composite score.

The four composite scores are verbal memory, visual memory, visual -motor processing speed, and reaction time. Table 2- 1 illustrates the ImPACT neurocognitive testing battery. A detailed description of individual tests and composite score has been detailed elsewhere..$^{59,63,136}$

ImPACT is one of the most studied computerized tools in the field of computerized neurocognitive testing. The validity, ${ }^{136-137}$ reliability, ${ }^{12,18,138}$ and sensitivity ${ }^{59}$ of ImPACT have been examined. ImPACT is primarily used in quantifying the neurocognitive and symptom outcomes after concussion. ${ }^{139} \mathrm{ImPACT}$ has also been used to examine the correlation between on- field markers of concussion severity and neurocognitive performance and symptoms following sports related concussion. ${ }^{62,} 65,140$ Moreover, ImPACT is used to examine if the presence of a certain symptom (e.g. headache, fogginess) is correlated with poorer neurocognitive performance. ${ }^{140-141}$ The successive and serial administration of ImPACT

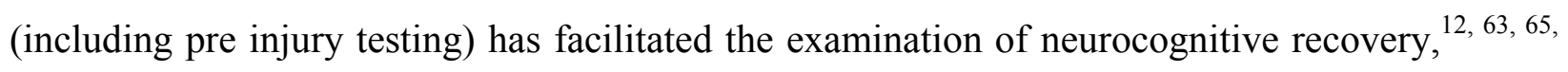
${ }^{139}$, symptoms reolution, ${ }^{18}$ and the presence of the cumulative effects of concussion. ${ }^{142-143}$ 
Table 2-1: The ImPACT Neuropsychological Testing Battery

\begin{tabular}{|c|c|}
\hline Test Name & Neurocognitive domain measured \\
\hline Word Memory & Verbal recognition memory (learning and retention) \\
\hline Design Memory & Spatial recognition memory (learning and retention) \\
\hline X's and O's & Visual working memory and cognitive speed \\
\hline Symbol Match & Memory and visual-motor speed \\
\hline Color Match & Impulse inhibition and visual-motor speed \\
\hline Three Letter Memory & Verbal working memory and cognitive speed \\
\hline Symptom Scale & Rating of individual self- reported symptoms \\
\hline Composite scores & Contributing scores \\
\hline Verbal Memory & $\begin{array}{l}\text { Word memory (learning and delayed), symbol match memory } \\
\text { score, three letter memory score }\end{array}$ \\
\hline Visual Memory & $\begin{array}{l}\text { Design Memory (learning and delayed), X's and O's percent } \\
\text { correct }\end{array}$ \\
\hline Reaction time & $\begin{array}{l}\text { X's and O's (average counted correct reaction time), symbol } \\
\text { match (average weighted reaction time for correct response) }\end{array}$ \\
\hline $\begin{array}{l}\text { Visual Motor Processing } \\
\text { Speed }\end{array}$ & $\begin{array}{l}\text { X's and O's (average correct distracters), symbol match } \\
\text { (average correct responses, three letter (number of correct } \\
\text { numbers correctly counted) }\end{array}$ \\
\hline
\end{tabular}




\subsubsection{Balance and posture}

Poor balance and postural instability have been reported in many studies post concussion. ${ }^{14,25-27,}$

29 However, the extent of balance disorders after concussion has not been clearly defined. Both static and dynamic balance has been examined after concussion. Static balance requires individuals to maintain balance in quiet stance (i.e. feet in place). Dynamic balance requires individuals to maintain balance while the body is moving (e.g. gait). While many of the early studies have examined the effect of concussion on static balance, recent literature has investigated the effect of concussion on balance in dynamic and dual task environments.

Regardless of the environment where balance was tested, it has been acknowledged that the variability in selected outcome measures and its psychometrics may contribute to the variability in the reported prevalence and course of recovery of balance disorders after concussion. ${ }^{15-16,29}$

Impairments in static balance have been examined using the modified protocol of Clinical Test of Sensory Interaction and Balance (CTSIB), Balance Error Scoring System (BESS), ${ }^{144}$ and SOT. Studies on the immediate effect of concussion on static balance reveal that patients exhibit increased postural sway ${ }^{14}$ and sway velocities in both coronal and sagittal planes. ${ }^{145}$ Patients also exhibit poorer performance on the Balance Error Scoring System (BESS). ${ }^{14,} 31$ However, most studies acknowledge that impairments in static balance usually resolve in the first 3-5 days after concussion. ${ }^{30-31,48,146-147}$

Dynamic postural instability has been correlated with dysfunction in sensory integration $^{148}$ or lack of attentional resources required for dynamic balance performance, ${ }^{29}$ and 
therefore dynamic postural stability is often examined when sensory conflict $^{148-149}$ or a dual $\operatorname{task}^{150-153}$ is introduced.

Studies of dynamic balance impairments after concussion revealed that patients exhibit slower gait velocity, ${ }^{28,}{ }^{151-152}$ shorter stride length, ${ }^{150}$ and wider step width. ${ }^{29}$ They adopt a conservative gait pattern exhibited by less sway and slower sway velocity in the sagittal plane. ${ }^{15}$ 28-29, 152 This conservative strategy is often interpreted as a compensatory mechanism for reduced postural control (i.e. more sway) in the coronal plane movement. ${ }^{152}$

Reports about the recovery of dynamic balance stability after concussion yield mixed results; while some studies on sensory conflicts revealed that the destabilization effect of visual field motion on balance may take 30 days to resolve, ${ }^{148}$ other reports demonstrated that patients with concussion may exhibit an inconsistent pattern of recovery, in which many aspects of gait stability are still impaired at four weeks after injury. ${ }^{151}$ This variability has been attributed in part to the variations in psychometric properties of different testing procedures and its ability to detect dynamic balance deficits at different times of the recovery process $;{ }^{15,29}$ for example, it has been suggested that dual cognitive tasks and obstacle crossing are better in detecting gait adaptations at different times of the recovery process; while attention divided gait is able to better distinguish gait deviations immediately following a concussion, obstacle crossing can be used further along in the recovery process to detect new gait adaptations. ${ }^{15}$

Other measures of gait and functional balance are used in vestibular and balance therapy post- concussion. These measures will be detailed below in the methods chapter. 


\subsubsection{Self- report symptoms}

The use of self- report symptoms has been widely acknowledged as a useful assessment tool for individuals with concussion. A self- report symptom checklist is the most common assessment tool employed by athletic trainers. ${ }^{154}$ Although a recent meta analysis has concluded the largest effect of concussion is in the domain of self- report symptoms compared to the neurocognitive and postural testing, ${ }^{19}$ there is a consensus that self- report symptoms should be incorporated in a multidisciplinary form of assessment, and the clinical decisions should not be solely based on the resolution of symptoms. ${ }^{84}$ Symptoms have been obtained through formal and informal evaluations. While the informal evaluations are sparse in the published literature, ${ }^{155}$ different formal symptom checklists have been published and used by a thletic trainers ${ }^{125,} 154$ and neuropsychologists. $^{18,140-141,156}$ These checklists are designed to quantify the severity and recovery of the self- report symptoms after concussion. ${ }^{18}$

Table 2-2: Clusters of self- report symptoms after concussion

\begin{tabular}{|l|l|l|l|}
\hline \multicolumn{1}{|c|}{ Physical } & \multicolumn{1}{|c|}{ Cognitive } & \multicolumn{1}{c|}{ Emotional } & \multicolumn{1}{c|}{ Sleep } \\
\hline Headache & Feeling mentally foggy & Irritability & Drowsiness \\
Nausea & Feeling slowed down & Sadness & Sleeping less than usual \\
Vomiting & Difficulty concentrating & More emotional & Sleeping more than usual \\
Balance problems & Difficulty remembering & Nervousness & Trouble falling a sleep \\
Dizziness & & & \\
Visual problems & & & \\
Fatigue & & & \\
Sensitivity to light & & & \\
Sensitivity to noise & & & \\
Numbness & & & \\
Tingling & & & \\
\hline
\end{tabular}

The comparison of evidence among the different checklists is challenged by considerable variation in their content, grading systems, ${ }^{19,} 157$ and the lack of reliability and other known 
psychometrics. ${ }^{18}$ The Post- Concussion Symptom Scale (PCS) is one of the most widely used checklists. It is a 22-item scale that was developed as a part of the Pittsburgh Steelers concussion management program. ${ }^{18}$ The PCS is designed to quantify the severity of symptoms in the acute phase of recovery after concussion, and is based on a 7 point Likert scale. For any particular symptom, the scale ranges from 0 (no symptom) to 6 ( severe), and the total PCS score is calculated by adding the scores obtained for the 22 items. The PCS has been examined for psychometric data, clinical interpretation and normative scores. ${ }^{18,138,141}$ The PCS is available in paper form and is incorporated in the ImPACT computerized neurocognitive testing program. ${ }^{24}$

The PCS has been used to quantify the self- report symptoms in patients after concussion in large number of studies, ${ }^{18,21,50,59,80,139-140,142,158-159}$ and has been adopted for use by the National Football League (NFL) and the National Hockey League (NHL). ${ }^{18}$

Other checklists include the Rivermead post concussion symptoms questionnaire (RPQ), ${ }^{160}$ and concussion symptoms survey (CSS). ${ }^{161}$ The Rivermead post concussion symptoms questionnaire is a 16 symptoms checklist. It uses a likert scale of five points between 0 (not experienced at all) and 4 (severe problem). The model has been developed, validated and assessed for reliability by King et al. ${ }^{160}$ Although one report has concluded the RPQ does not meet the modern psychometric standards and should not be used in its current form, ${ }^{162}$ the Rivermead post concussion questionnaire has been used for individuals with post concussion in many studies. ${ }^{163-170}$

The prevalence of self- report symptoms after concussion is variable across different reports. Many factors may have contributed to the large degree of variability seen; there have been many proposed operational definitions of concussion and therefore the selection of participants is influenced by wh is defined as "concussion". ${ }^{108}$ Moreover, the recruitment 
process may influence the sample characteristics of selected participants and inflate the prevalence of self- report symptoms. ${ }^{108}$ Participants in some studies were obtained from the registry in emergency departments. ${ }^{109,170-172}$ The longitudinal reports of self- report symptom recovery tend to bias toward the more severe spectrum of patients that are presented with unresolved symptoms and seen for follow up. ${ }^{108,171}$ Table 2-3 illustrates the total symptom score reported by many studies at the initial evaluation after concussion and at other times.

Table 2-3: Total symptoms score reported after concussion

\begin{tabular}{|c|c|c|c|c|c|c|}
\hline Study & $\mathrm{n}$ & Scale & Mean age & $\begin{array}{c}\text { Mean } \\
\text { initial } \\
\text { total } \\
\text { score }\end{array}$ & $\begin{array}{c}\text { Follow } \\
\text { up(days) }\end{array}$ & $\begin{array}{c}\text { Mean total } \\
\text { score at } \\
\text { follow up }\end{array}$ \\
\hline McCrea et al, $^{31}$ & 94 & $\begin{array}{c}\text { Graded } \\
\text { Symptom } \\
\text { checklist }\end{array}$ & 20 y.o & 20.93 & 90 & .6 \\
\hline Lovell et al, $^{65}$ & 64 & $\begin{array}{c}\text { Post concussion } \\
\text { scale (PCS) }\end{array}$ & $\begin{array}{c}\text { High } \\
\text { school } \\
\text { athletes }\end{array}$ & 25 & 7 & 7 \\
\hline $\begin{array}{c}\text { Register- } \\
\text { Mihalik, }\end{array}$ & 392 & $\begin{array}{c}\text { Graded } \\
\text { symptom } \\
\text { checklist }\end{array}$ & 17 y.o & 11.70 & 7 & 1.9 \\
\hline
\end{tabular}

$\mathrm{n}=$ Number of participants

Post- traumatic headache is the most common symptom after concussion. ${ }^{171,174-175}$

Prevalence of initial headache after concussion has been reported to range between $43 \%$ to $86 \%$ of patients (Table $2-4){ }^{18,171,176}$ Persistent headache is also reported in a period up to three months after concussion. ${ }^{170,172}$

Although post traumatic headache can exist in many forms such as tension-like, cluster like or mixed headaches, ${ }^{175}$ individuals with migraine headache are found to have greater neurocognitive deficits compared to individuals with other forms of headache and individuals with no he adache. ${ }^{158}$ Migraine is also found to be an independent risk factor for sustaining concussion. $^{177}$ 
The prognostic value of headache has been investigated in many studies. ${ }^{140,158,163,173,178-}$ ${ }^{180}$ Evidence from a clinical population of mTBI revealed that the presence of headache at time of admission to the emergency department is associated with development of post concussion symptoms at one and six months after injury. ${ }^{163,178}$ Individuals with headache are found to have worse neurocognitive and balance testing, and found to report more symptoms than individuals who do not have headache after concussion; Collins et al. revealed a correlation between the neurocognitive testing scores and the severity of headache post concussion. ${ }^{140}$ Individuals with Post-traumatic Migraine to have worse neurocognitive performance compared to athletes who did not have headache or have other types of headache after sport related concussion. ${ }^{158}$

Table 2-4: Prevalence of Headache after concussion

\begin{tabular}{|c|c|c|c|c|c|}
\hline $\begin{array}{c}\text { Study } \\
\text { (sample size) }\end{array}$ & $\begin{array}{l}\text { Mean age } \\
\text { (years) }\end{array}$ & $\begin{array}{l}\text { Outcome } \\
\text { measure }\end{array}$ & $\begin{array}{l}\% \text { report initial } \\
\text { symptoms } \\
\text { (mean } \\
\text { severity) }\end{array}$ & $\begin{array}{l}\text { Time of follow } \\
\text { up }\end{array}$ & $\begin{array}{c}\text { \% reporting } \\
\text { symptom at } \\
\text { follow up } \\
\text { (mean severity) }\end{array}$ \\
\hline Blinnman et al, $_{(116)}^{171}$ & 14.1 & PCS & $71.6(2.7)$ & 2-3 weeks & $31.8(1.8)$ \\
\hline $\begin{array}{l}\text { Collins et al, } \\
\text { (109) }\end{array}$ & 15.8 & PCS & $\mathrm{NR}^{\dagger}$ & 1 week & $33.0(2.7)$ \\
\hline $\begin{array}{l}\text { Faux et al, } \\
(100)\end{array}$ & 33.6 & RPQ & 100 & $\begin{array}{l}1 \text { month } \\
3 \text { months }\end{array}$ & $\begin{array}{l}30.4(\mathrm{NR})^{\dagger} \\
15.4(\mathrm{NR})^{\dagger}\end{array}$ \\
\hline 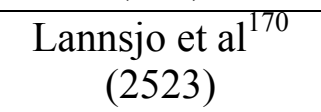 & 31 & RPQ & 43.2 & 3 months & $22(2.6)$ \\
\hline $\begin{array}{l}\text { Lovell et al, } \\
\quad(52)\end{array}$ & 16.8 & PCS & 88.5 & $\begin{array}{l}\text { Between } 1 \& 4 \\
\text { weeks }\end{array}$ & $32.7(\mathrm{NR}) \dagger$ \\
\hline $\begin{array}{c}\text { Savola et al, }{ }^{176} \\
\text { (37) }\end{array}$ & 33.7 & $\begin{array}{c}\text { Modified } \\
\text { version of } \\
\text { PRQ }\end{array}$ & 65 & 4 weeks & $38(\mathrm{NR})^{\dagger}$ \\
\hline
\end{tabular}

$\dagger$ NR: Not Reported 
Dizziness is also a frequent symptom after concussion. Twenty three to eighty one percent of persons post concussion report dizziness in the first days post concussion (Table 25). ${ }^{18,181}$ Of the $61 \%$ who reported dizziness in the initial days after concussion in one study, $41 \%$ reported mild dizziness, $16 \%$ reported moderate dizziness, and $4 \%$ reported severe dizziness. ${ }^{18}$ Estimates of the prevalence of persistent dizziness after mTBI varies widely from $16-18 \%$ at three months, ${ }^{170,181} 1.2 \%$ at 6 months ${ }^{182}$ to $32.5 \%$ at five years. ${ }^{183}$

The prognostic value of dizziness in mTBI was examined in many studies. ${ }^{163,176,178}$ In a clinical population 208 adults with mild and moderate brain injury, Chamelian et al reported that patients with dizziness were more symptomatic and demonstrated worse psychosocial functioning six months after injury compared to the patients who did not have dizziness. ${ }^{163}$

Although the results of this study may be confounded by the presence of moderate TBI patients in the sample, the results are consistent with other studies that investigated the role of dizziness in the outcomes after mTBI. Two studies have reported that dizziness at the time of admission to the ER after mild TBI is associated with the severity of post concussion symptoms at one and six months after injury. ${ }^{176,178}$ Dizziness was also linked to psychological distress at 6 months after injury and found to be an independent factor for failure to return to work after mild to moderate head injury. ${ }^{163}$

Balance problems are af requent symptom after concussion. Self- report balance problems have been reported in as high as $60 \%$ after concussion. ${ }^{171}$ Persistent self report balance problems have also been reported after one week of concussion (Table 2-6). ${ }^{18,171}$ Although some studies have suggested that self- reported balance problems usually resolve in the first days after concussion, ${ }^{31,144,146}$ patients may exhibit decreased balance performance despite not reporting balance problems. ${ }^{184}$ 
Table 2-5: Prevalence of Dizziness after concussion

\begin{tabular}{|c|c|c|c|c|c|}
\hline $\begin{array}{c}\text { Study } \\
\text { (sample size) }\end{array}$ & $\begin{array}{c}\text { Mean age } \\
\text { (years) }\end{array}$ & $\begin{array}{c}\text { Outcome } \\
\text { measure }\end{array}$ & $\begin{array}{c}\text { \% report initial } \\
\text { symptoms } \\
\text { (mean severity) }\end{array}$ & $\begin{array}{c}\text { Time of follow } \\
\text { up }\end{array}$ & $\begin{array}{c}\text { \% reporting } \\
\text { symptom at follow } \\
\text { up (mean severity) }\end{array}$ \\
\hline $\begin{array}{c}\text { Blinmann et al, }{ }^{171} \\
(116)\end{array}$ & 14.1 & PCS & $60.3(2.7)$ & $2-3$ weeks & $27.0(1.6)$ \\
\hline $\begin{array}{c}\text { Broglio et al, }{ }^{21} \\
(32)\end{array}$ & 19.7 & PCS & $28.1(.75)$ & NA $\ddagger$ & NA \\
\hline $\begin{array}{c}\text { Lovell et al, } \\
(52)\end{array}$ & 16.8 & PCS & 78.8 & $\begin{array}{c}\text { Between 1 and } \\
4 \text { weeks }\end{array}$ & $17.3(\mathrm{NR}) \dagger$ \\
\hline $\begin{array}{c}\text { Lannjo at al, }{ }^{170} \\
(2523)\end{array}$ & 31 & PRQ & 31 & 3 months & $16(2.6)$ \\
\hline $\begin{array}{c}\text { Savola et al, } \\
(37)\end{array}$ & 33.7 & $\begin{array}{c}\text { Modified } \\
\text { version of } \\
\text { RPQ }\end{array}$ & 49 & 4 weeks & $43(\mathrm{NR}) \dagger$ \\
\hline
\end{tabular}

$\dagger$ NR: Not Reported; $\$ N A$ : Not applicable; RPQ: The Rivermead Post- Concussion symptom questionnaire

Table 2-6: Prevalence of Balance problems after concussion

\begin{tabular}{|l|c|c|c|c|c|}
\hline \multicolumn{1}{|c|}{$\begin{array}{c}\text { Study } \\
\text { (sample size) }\end{array}$} & $\begin{array}{c}\text { Mean age } \\
\text { (years) }\end{array}$ & $\begin{array}{c}\text { Outcome } \\
\text { measure }\end{array}$ & $\begin{array}{c}\text { \% report initial } \\
\text { symptoms } \\
\text { (mean severity) }\end{array}$ & Time of follow up & $\begin{array}{c}\text { \% reporting symptom } \\
\text { at follow up (mean } \\
\text { severity) }\end{array}$ \\
\hline $\begin{array}{l}\text { Blinmann et al, }{ }^{171} \\
(116)\end{array}$ & 14.1 & PCS & $60.3(2.6)$ & $2-3$ weeks & $25.4(1.5)$ \\
\hline $\begin{array}{l}\text { Broglio et al, }{ }^{21} \\
(32)\end{array}$ & 19.7 & PCS & $34.4(.75)$ & NA & NA \\
\hline $\begin{array}{l}\text { Lovell et al, } \\
(52)\end{array}$ & 16.8 & PCS & 55.8 & $\begin{array}{c}\text { Between } 1 \text { and } 4 \\
\text { weeks }\end{array}$ & $11.5(\mathrm{NR}) \dagger$ \\
\hline
\end{tabular}

$\dagger$ NR : Not reported; NA: Not applicable; PCS; Post- Concussion Symptom scale

Many have suggested that the symptoms after concussion could be clustered into broad constructs. $^{24,185-186}$ Although the nomenclature is slightly different across reports, the dividing of symptoms into clusters is suggested to offer a better description about the effect of concussion and reduce the redundancy and confounding in the symptom checklists. In one study, Piland et al. have suggested that grouping of the head injury scale (HIS) of symptoms into a model of three constructs (somatic, cognitive and neurocognitive) will demonstrate factorial and construct 
validity among collegiate athletes. ${ }^{185}$ Others have suggested that the symptoms could be divided into somatic, cognitive, neuropsychiatric or affective constructs. ${ }^{24}$ In a recent report, the CDC has noted that the symptoms of concussion generally fall into four categories: physical (i.e. somatic), cognitive, emotional (i.e. mood), and sleep (Table 2-2). ${ }^{186}$ However, the current classification by the CDC reflects consensus among group of experts and is not based on empirical evidence and therefore the use of the clusters is mainly for descriptive purposes.

Additionally, it is unclear if the current categorization has any clinical implications since the symptom evaluation tools that are currently in use do not have categories sub scores.

\subsection{MULTIFACATED RECOVERY PROCESS AFTER CONCUSSION}

\subsubsection{Predictors of recovery after concussion}

\subsubsection{Initial markers of concussion severity}

The following signs have been recognized as the hallmarks of concussion severity; loss of consciousness (LOC), amnesia, and disorientation. Many concussion severity scales have relied on these signs for the classification of severity, especially the LOC. ${ }^{54,187}$ However, recent research has doubted the prognostic value of these markers, and whether they represent valid measures to determine the severity and therefore the course of recovery after concussion. ${ }^{61-62,77 \text {, }}$ 188

Loss of consciousness may be associated with early neurocognitive deficit, but not prolonged neurocognitive deficit. ${ }^{77}$ McCrea et al. found that despite the normal neurocognitive scores on the Standardized Assessment of Concussion (SAC), patients with LOC are more 
symptomatic than patients who did not have LOC 48 hours after concussion. ${ }^{77}$ Lovell et al. have retrospectively examined the relationship between neurocognitive performance and the presence of LOC after concussion in 383 patients. The study demonstrated no significant differences in the neurocognitive testing between patients who sustained LOC vs. no LOC. ${ }^{61}$ The results of this study are consistent with other studies that have examined the prognostic value of LOC on the prolonged neurocognitive performance. ${ }^{62,}{ }^{188}$ However, Auspland et al. has found that athletes with LOC demonstrated prolonged return to play after concussion. It is unclear whether these findings are attributed to providers' conservative care or prolonged actual deficit. ${ }^{180}$ In summary, the use of LOC as a marker of concussion severity has been scrutinized by many studies and

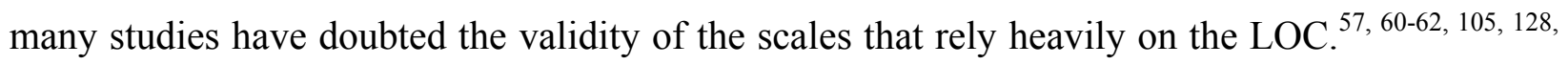
$189-191$

Other on-field markers have been also investigated for prognostic value. ${ }^{191}$ Collins et al. reported increased risk of reduced memory scores and prolonged symptoms with the presence of retrograde amnesia, anterograde amnesia, and disorientation. ${ }^{62}$ The duration of on-field mental status changes such as retrograde amnesia and confusion were found to be related to poor memory scores and higher symptoms at 36 hours, 4 and 7 days after concussion. ${ }^{65}$ Others have found that post traumatic amnesia (PTA) is correlated with worse neurocognitive outcomes at day 2 a fter concussion. ${ }^{189}$ These results suggested on-field mental status appeared to have a

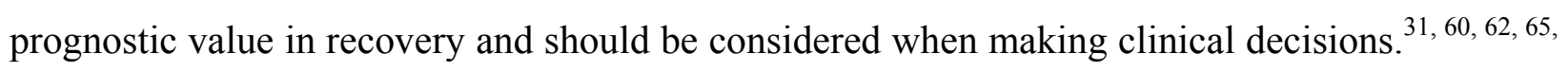
$70,187,190$ 


\subsubsection{Age}

Many have hypothesized that there are differences between children and adults that may affect concussion management. ${ }^{71,192}$ Many psychosocial factors could affect a children's recovery such as academic and social issues that need to be accounted for in the management process. ${ }^{192}$

Children also change in their developmental abilities and these changes warrants frequent updates to baseline neurocognitive testing. ${ }^{4}{ }^{125,}{ }^{193-194}$ A differential rate of recovery between children and adults is seen in many studies. ${ }^{64-66,110,139,190}$ Many pathophysiological factors may contribute to differential rates of recovery of children after concussion. Children's tolerance to biochemical changes associated with concussion may be different from adults, and consequently the sequelae from impacts of the same magnitude may be different between children and adults. ${ }^{4}$ 195

Differences in glutamate sensitivity and prolonged diffuse cerebral swelling in children post injury were some of the factors suggested to contribute to the protracted recovery pattern seen in children. ${ }^{57,192,195-196}$ These metabolic factors are thought to further expose children to subsequent injury that may have catastrophic consequences such as Second Impact Syndrome (SIS). ${ }^{194,197}$

Many studies have noted that high school athletes are more susceptible to concussive injuries $^{60,63,128,197}$ and have a prolonged recovery pattern compared to older athletes in the domains of neurocognitive testing and symptom resolution. ${ }^{64-66,139,190}$ For instance, while the memory function in college athletes returned to the level of matched controls by day three post concussion, high school athletes needed 7 days to return to normal memory function. ${ }^{64} \mathrm{~A}$ similar pattern of prolonged memory dysfunction in high school athletes was also noted by Sim et al. ${ }^{66}$ 
Despite the differential rate of recovery shown by studies, none of the standardized concussion assessment scales has provided age specific guidelines, and therefore many have doubted the use of standardized grading scales in children. ${ }^{57,60,63-64,110}$

There is a consensus that conservative management strategy should be used with children post concussion. ${ }^{192-193}$ This conservatism is endorsed by the National Athletic Trainers Association and the $3^{\text {rd }}$ International Conference on Concussion in Sport. ${ }^{70,84}$

Finally, it is noteworthy that all the age- related studies have used measures of neurocognitive testing and self- report symptoms. None of age-related studies described the effects of age on recovery of impairments in balance and posture or if there is a differential rate of recovery between children and adults for balance disorders.

\subsubsection{Gender}

The studies on the effect of gender on $\mathrm{c}$ oncussion incidence have yielded mixed results. Although some studies have found females to have a higher incidence of concussion, ${ }^{174,}$ 198-200 other studies found equivalent concussion rates in both genders. ${ }^{79,201-202}$

Differences between genders in baseline neurocognitive testing were found in some studies. ${ }^{203-204}$ Barr et al. has found that while male athletes perform better on tests of visual memory, female athletes perform better on verbal memory tests. ${ }^{203}$ It has been also found that females may experience different effects after concussion compared to males and may experience a different course of recovery. ${ }^{67-68,204}$ Females have shown greater decline in their memory scores, ${ }^{67}$ reaction time and processing speed. ${ }^{68-69,} 122$ Females also have demonstrated worse overall self- report symptoms, ${ }^{68-69,93,167,201,205-206}$ and were found to report headache, sleep disturbances and depression more than male counterparts. ${ }^{205,207}$ 
Despite the studies demonstrating different recovery patterns in self- report symptoms and neurocognitive testing, there were no studies to examine recovery in the balance and posture domain of assessment based on gender

\subsubsection{Concussion History}

The cumulative effect of concussion history on neurocognitive performance and self- report symptoms have been investigated by many studies. Iverson et al. reported that individuals with previous concussion history are more symptomatic and have worse neurocognitive performance on pre- season testing. ${ }^{143}$ Others have suggested that a history of concussion increases the risk for sustaining subsequent concussion by up to 5.8 times. $^{69,208-211}$

The cumulative effect of concussion also has been studied by comparing performance and recovery between individuals who have a previous history of concussion and individuals who sustain their first concussion. The effect of three or more previous concussions on a subsequent concussion yield convincing results. Athletes with three or more previous concussions were reported to be 9.3 times more likely to experience more markers of concussion

severity, ${ }^{208} 7.7$ times more likely to experience major decline in memory performance, ${ }^{143}$ and were found to report more symptoms of subsequent concussions. ${ }^{212}$ The evidence for the effect of one or two previous concussions is mixed. While some studies suggested that a history of one or two concussions have a cumulative effect, ${ }^{69,155}$ many studies have not found such an effect. ${ }^{142,}$ 213

Belanger et al. have noted that the effect size of concussion on neurocognitive performance was different between the studies that excluded individuals with previous concussions ( $\mathrm{d}=.11)$ and the studies that did not exclude individuals with previous concussion 
$(\mathrm{d}=.74) .{ }^{46,}{ }^{214}$ Most studies analyzed the cumulative effect of concussion on $t$ he overall neurocognitive functioning. However, Belanger et al. found that a history of multiple concussions is associated with worse performance for the measures of executive functioning and delayed memory. ${ }^{214}$

\subsubsection{Correlation between outcome measures among different domains during recovery}

In examining the recovery process after concussion, researchers have often viewed the recovery process from different points of view; Subjective vs. objective and cognitive vs. motor recovery.

The diversity in these points of view have enhanced our understanding of the multifaceted nature of the recovery process after concussion, and improved the management of patients with concussion. Nonetheless, despite the improvements made in the validation of different assessment domains, no single one should be a stand alone instrument. ${ }^{16,23,60,107,110}$

Using multiple domains of assessment that include neurocognitive testing, self- report symptoms and postural testing is found to increase the sensitivity of a concussion assessment battery to exceed $90 \%$ compared to $79 \%$ obtained by the most sensitive instrument only

(ImPACT). ${ }^{20}$ In another study, Van Kampen et al. reported that the use of neurocognitive testing (i.e. ImPACT) in addition to self- report symptoms resulted in a 19\% net increase in sensitivity compared to self- report symptoms alone. ${ }^{215}$

The multi-faceted nature of concussion effects and the recovery process may shed light on the relationships between the different domains of assessment during the recovery process.

Surprisingly, the recovery process has been studied separately within each domain, and little research was directed to the interrelationships between different domains. 
Recent research in laboratory settings suggested that the performance of cognitive and postural control tasks simultaneously will adversely affect the performance of both tasks. ${ }^{29,150-151}$ When clinical outcome measures are used to examine the relationship between different domains, the evidence is less clear. Few studies have concluded that cognitive and motor effects of concussion resolve differently after concussion. ${ }^{16,216}$ Parker et al. have found a differential rate of recovery by comparing the recovery pattern in ImPACT composite scores and different dynamic motor tasks. ${ }^{216}$ The lack of correlation between measures of different domains has sparked a big debate. For instance, while some view the lack of perfect correlation between performance measures and self- report symptoms as an indication for the lack of sensitivity in performance measures ${ }^{48}$ or an indication for the inaccuracy of self- report symptoms, ${ }^{21}$ others speculate that they may represent fundamentally different neurobehavioral processes, and therefore they should not be expected to correlate in all cases. ${ }^{14,20,104}$ Lovell et al. has concluded that post concussion symptoms are a result of combinations of neurocognitive deficits and other factors (e.g. vestibular dysfunction), and therefore the correlation (or lack thereof) between symptoms and neurocognitive testing is expected to be less than perfect. ${ }^{104}$

Despite the conceptual debate about the reasons behind the correlation (or lack thereof) between measurements from different domains, examining the correlation may enhance our understanding about the multi-faceted nature of concussion effects and recovery. Preliminary evidence from self- report symptoms revealed that balance problems were significantly correlated with feeling mentally foggy, difficulty remembering, and difficulty concentrating. ${ }^{21}$

Recent work by B roglio et al. examined if the symptoms of feeling mentally foggy, difficulty concentrating, and difficulty remembering are associated with decreased cognitive performance (i.e. ImPACT composite scores). The results revealed a significant correlations 
between feeling mentally foggy and reaction time, difficulty concentrating and verbal memory score and between difficulty remembering and change in verbal memory composite score and

reaction time. ${ }^{21}$ In the same study Broglio et al. examined the relationship between self- report "dizziness" and "balance problems" and Sensory Organization Test (SOT). The results demonstrated a significant correlation between dizziness and change in the vestibular ratio of SOT. The study also found a significant correlation between change in the total score of SOT, change in the three ratios of SOT, and balance problems. ${ }^{21}$

In summary, preliminary evidence suggests the presence of a correlation between selfreported symptoms related to cognition and neurocogntive testing performance. Also, a correlation was found between self- reported symptoms related to balance and balance performance. However, the evidence is less clear when the comparison is made between neurocognitive testing and balance performance.

\subsection{VESTIBULAR REHABILITATION FOR PATIENTS POST CONCUSSION}

\subsubsection{Evidence behind vestibular rehabilitation for concussion}

The role of the vestibular system in balance and posture has been extensively studied and numerous studies have found that impairments in vestibular function may lead to balance disorders and dizziness. ${ }^{217-225}$ Although the effects are variable, ${ }^{226}$ many studies have demonstrated that vestibular rehabilitation can help reduce dizziness and balance disorders. ${ }^{26-231}$

Post- traumatic dizziness (e.g. after concussion) is also suggested to be associated with impairments in the peripheral vestibular system. ${ }^{42-43,}$ 146-147, 232-233 Balance disorders after 
concussion have also been attributed to dysfunction in sensory integration in which the vestibular system is a key part of normal functioning..$^{34,42-43}$ Vestibular rehabilitation exercises may reduce the dizziness and balance disorders after concussion. ${ }^{34,44}$

Despite the high incidence of dizziness and balance dysfunction in people who have had a concussion, reports of vestibular and balance rehabilitation in the management of concussion are sparse. ${ }^{34,44,234-235}$ Previous studies have shown that vestibular rehabilitation reduces dizziness and improves overall balance for individuals with head injury. ${ }^{39,44,236-237}$ The studies have also emphasized that post traumatic dizziness can be caused by a wide array of dysfunctions in the vestibular system, and therefore a customized impairment-based vestibular rehabilitation program is recommended to successfully reduce the complaint of dizziness and improve balance after concussion. ${ }^{33-34,38,238}$ Gurr et al. reported that vestibular rehabilitation comprised of graded exposure to head and body movements, anxiety management, coping strategies and education reduced complaints of vertigo and dizziness, and improved the balance of individuals standing on an unstable surface. ${ }^{44}$ In another study, Hoffer et al. reported that in patients with mild TBI, vestibular rehabilitation exercises consisting of an individualized program of somatosensory exercises combined with aerobic activity, vestibuolo-ocular reflex, and cervico-ocular reflex activities reduced complaints of dizziness and accelerated return to work. ${ }^{239}$ Although the use of vestibular rehabilitation in the treatment of concussion-related dizziness and balance dysfunction is promising, these studies were limited because of small sample size. It is also unclear if the improvement made over the course of vestibular rehabilitation is due to the intervention or due to the natural course of recovery. Without control group, it is difficult to attribute the improvement to the provided intervention. 


\subsubsection{Outcome measures used in vestibular rehabilitation}

The vestibular physical therapist uses many outcome measures to track recovery and perceived improvement over the course of vestibular rehabilitation. These measures can be classified into self- report and performance measures.

It has been reported that there could be a d iscrepancy between self- report and performance measures of neurocognitive and balance functioning after concussion. ${ }^{21,}{ }^{184}$ The recent recommendations on concussion management have emphasized the use of both selfreport and performance measures to track the recovery of patients post concussion. For post concussion patients treated with vestibular rehabilitation, it is not uncommon for the vestibular physical therapist to employ a wide array of self- report and performance measures to track

recovery and make recommendations regarding discharge or return to work or practice. ${ }^{43}$ Selfreport and performance measures used in the dissertation research will be reviewed in the Methods.

\subsubsection{Limitations of previous work and rationale for proposed studies}

The vestibular physical therapist can play a key role in a multidisciplinary management of concussion by having a wide variety of assessment tools that cover multiple domains and by providing an intervention that appears to be effective based on the small number of published studies to date. However, in a multidisciplinary environment of concussion management, vestibular rehabilitation lags behind other interventions used in concussion management. In order for vestibular rehabilitation to be involved in an evidence-based medicine and cost driven health care system, the limitations that surround the use vestibular rehabilitation in patients with 
concussion need to be addressed. By overcoming the limitations mentioned below, vestibular rehabilitation may have a greater role to play in the management of patients with concussion.

\subsubsection{Rationale for the first study}

Different gait and functional balance measures have been used to evaluate and re- assess the gait and balance dysfunction in patients with vestibular disorders including patients with postconcussion balance dysfunction. These measures are used as an indicator of the degree of balance impairment after concussion, and the serial administration of these measures is used to track the recovery of gait and balance function.

Many of the clinical decisions regarding the exercise initiation / progression or discharge from vestibular rehabilitation therapy are often based on the scores obtained through the gait and balance testing as well as symptom resolution. However, most of these measures were initially developed to be used in middle aged and older adults and no study has investigated the reliability and the normative scores of these measures in children between the age of 14 and 18. By providing specific normative scores for this age group, we will be able to use these normative scores to determine the impact of concussion on children's balance performance and it will allow us to track the recovery process in children after concussion. The purpose of this study is to provide normative data and to examine the reliability for clinical gait/ balance measures for healthy high school aged children.

\subsubsection{Rationale for the second study}

The evidence behind vestibular rehabilitation in concussion management is sparse and largely based on clinical experience and a small number of studies with small sample size and limited 
methodological design. Dizziness and imbalance reported by pe ople who were referred for vestibular rehabilitation after concussion has not been described.

It has not been established if the effects of concussion regarding dizziness and balance dysfunction are the same for children and adults; children's tolerance to biochemical changes associated with concussion may be different from adults, and consequently the sequelae from impacts of the same magnitude may be different between children and adults. ${ }^{4}$ In addition, the role that is played by the continuous and rapid maturation in children's cognitive abilities and postural strategies in the recovery process is unknown. ${ }^{4,64,240-241}$ Despite all of these factors, the above mentioned studies have not described whether the amount of recovery made in vestibular rehabilitation was different between children and adults. ${ }^{4242-243}$

The second study will describe the severity of dizziness and balance dysfunction in patients who were referred to vestibular rehabilitation after being diagnosed with concussion and describe the outcomes of vestibular rehabilitation on dizziness and balance dysfunction after concussion, and to test whether the amount of recovery is similar between children and adults.

\subsubsection{Rationale for the third study}

Although the individualized nature of the vestibular rehabilitation program for individuals after concussion has been emphasized, ${ }^{244}$ an understanding of which specific exercises have been prescribed for this population may be useful for several reasons. Assuming that the physical therapists adhered to the problem-oriented approach described in vestibular rehabilitation practice guidelines,${ }^{244}$ the study can provide a detailed picture of the specific impairments and functional limitations encountered by the individuals with concussion and the path by which they returned to their pre-morbid status. Moreover, by understanding the prescription and progression 
patterns provided by expert clinicians, physical therapists new to prescribing vestibular rehabilitation exercises for patients with concussion can use this information to better understand how to start and then make the exercises more challenging. Additionally, therapists can use the framework of exercise categories, exercise groups and exercise modifiers to better document the detailed prescription pattern of vestibular rehabilitation exercises.

In the previous proposed aim, a r eduction in dizziness and improvement in balance occurred after patients with concussion received a v estibular rehabilitation exercise program consisting of gaze stabilization, standing balance, and walking exercises. Consequently, the purpose of this study is to describe exercise prescription patterns in patients treated with vestibular rehabilitation exercises after concussion. The analysis will describe the progression of the exercises and common exercise volumes for vestibular rehabilitation exercises used in the management of individuals with dizziness and balance disorders after concussion.

\subsubsection{Rationale for the fourth study}

In examining the recovery process after concussion, clinicians and researchers assess a variety of signs, symptoms, and physiological functions (e.g. sensory, cognitive, and motor). ${ }^{21,62,80,124,141}$ The diversity in measurements used to assess individuals with concussion has enhanced our understanding of the multi-faceted nature of the effects after concussion, and improved the sensitivity of the assessment battery available to record functioning in individuals with concussion. ${ }^{20}$ A few studies have concluded that cognitive and motor effects of concussion resolve differently after concussion. ${ }^{16,216}$ Parker et al. have found a differential rate of recovery by comparing the recovery pattern in ImPACT composite scores and different dynamic motor tasks. $^{216}$ 
The lack of correlation between recovery of measures of different domains has been a subject of debate. ${ }^{14,20-21,48,104}$ For instance, while some view the lack of perfect correlation between performance measures and self- report symptoms as an indication for the lack of sensitivity in performance measures ${ }^{48}$ or an indication for the inaccuracy of self- report symptoms, ${ }^{21}$ others speculate that they may represent fundamentally different neurobehavioral processes, or exhibit different recovery trajectories, and therefore they should not be expected to correlate in all cases. ${ }^{14,20,104}$ Lovell et al. has concluded that post-concussion symptoms are a result of combinations of neurocognitive deficits and other factors (e.g. vestibular dysfunction), and therefore, the correlation (or lack thereof) between symptoms and neurocognitive testing is expected to be less than perfect. ${ }^{104}$

Despite the conceptual debate about the reasons behind the correlation (or lack thereof) between measurements from different domains, examining the relationship among measurements may enhance our understanding about the multi-faceted nature of concussion effects and recovery. Collins et al. revealed a correlation between the neurocognitive testing scores and the severity of headache post concussion. ${ }^{140}$ Preliminary evidence from a study that examined selfreport symptoms revealed that "balance problems" were significantly correlated with "feeling mentally foggy", "difficulty remembering", and "difficulty concentrating". ${ }^{21}$ In addition, these symptoms were also associated with decreased cognitive performance (i.e. ImPACT composite scores). In the same study Broglio et al. examined the relationship between self- report "dizziness" and "balance problems" and the Sensory Organization Test (SOT). The study found a significant correlation between change in the total score of the SOT to balance problems. ${ }^{21}$

The purpose of this study is to examine the relationship between self-reported symptoms, neurocognitive performance and balance performance in individuals referred to vestibular 
physical therapy after concussion, and to examine the relationship between recovery in gait and balance measures and neuropsychological recovery made over the course of vestibular physical therapy. 


\subsection{FIRST AIM}

\subsection{INTRODUCTION}

The awareness of concussion injuries in high school aged children has increased substantially in the last decade. ${ }^{64,140,174,208,245}$ Many studies have noted that high school athletes are more susceptible to concussive injuries compared with older athletes. ${ }^{60,63,128,197}$ Furthermore, high school athletes may have prolonged recovery compared to older athletes in the domains of neurocognition and symptom resolution. ${ }^{64-66,139,190}$ Memory function in college athletes has been reported to return to the level of matched controls by day three post concussion whereas high school athletes required seven days to return to normal memory function. ${ }^{64}$ Differences between children and adults in glutamate sensitivity, tolerance to biomechanical changes after injury and different psychosocial factors have been proposed to explain the different courses of recovery

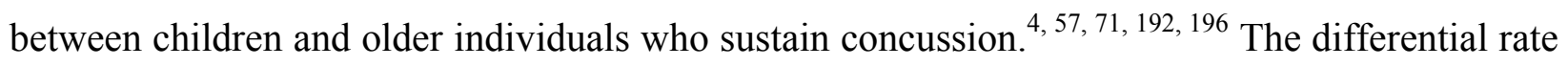
of recovery between children and adults led to a consensus that conservative management should be used with children post-concussion. ${ }^{70,84,192-193}$ One of the principles of conservative management includes not allowing return to play until the student-athlete achieves baseline (i.e. pre-concussion) performance on t ests of neurocognitive function. In cases where baseline performance measures do not exist, age-referenced normative scores for neuropsychological testing are used to help assist in making decisions about return to play. ${ }^{18,}{ }^{246}$ Additionally, 
maturational changes in neurcognitive performance may dictate use of the age-referenced scores if baseline testing has not been conducted recently., 125, 193-194, 247

Reports of dizziness and imbalance are prevalent in individuals who have had a concussion. ${ }^{18,}$ 170-171, 181-182 Estimates of the prevalence of persistent dizziness after mild traumatic brain injury (mTBI) (i.e. concussion) varies between $1.2 \%$ to $32.5 \%$ depending on age group and time of follow-up. ${ }^{170,181}{ }^{18,21,176,182}$ Persistent balance problems have also been reported after concussion. ${ }^{18,21,171}$ Vestibular rehabilitation is increasingly being used to manage dizziness and balance disorders resulting from vestibular system dysfunction. ${ }^{226-231}$ Previous studies have shown that vestibular rehabilitation may reduce dizziness and improve overall balance for individuals with head injury. ${ }^{33-34,39,44,248-249}$ Additionally, a recent report reported that children with dizziness and balance disorders after concussion appear to benefit from vestibular rehabilitation. ${ }^{249}$

For individuals treated with vestibular rehabilitation after concussion, it is common for the physical therapist to employ a wide array of self- report and performance measures to track recovery and make recommendations regarding discharge or return to work or athletic

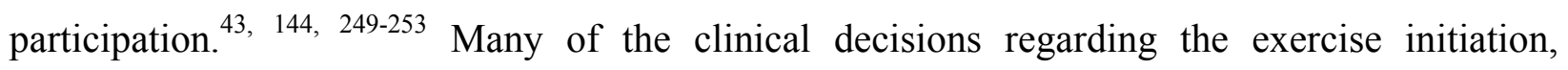
progression or discharge from physical therapy are based on the scores obtained through gait and balance testing as well as presenting symptoms. Adolescents present a challenge for balance assessment because they are too old for common developmental motor scales such as the Bruininks - Oseretsky Test of Motor Proficiency (4.5 -14.5 years). ${ }^{254}$ Furthermore, there are no estimates of reliability or normative scores in other common outcome measures utilized in vestibular physical therapy that were developed for use in middle-aged and older adults, such as the Activities - specific Balance Confidence (ABC) scale, ${ }^{255}$ the Dynamic Gait Index (DGI), ${ }^{256}$ 
the Functional Gait Assessment (FGA), ${ }^{257}$ gait speed (GS), the Timed "UP \&GO" (TUG), ${ }^{258}$ and the Five Times Sit to Stand (FTSTS) test. ${ }^{259}$ By providing specific normative scores for this age group, we will be able to quantify balance impairments in children after concussion and assist clinicians in making a better determination of when children are able to return to athletic participation after concussion. Therefore, the purpose of this study is to provide normative scores and determine the reliability of common clinical balance outcome measures in healthy children between the ages of 14 to 18 years.

\subsection{METHODS}

\subsubsection{Design}

A cross-sectional sample of students in the $9^{\text {th }}$ through the $12^{\text {th }}$ grades was obtained through local high schools. The study protocol was approved by the Institutional Review Board (IRB) at the University of Pittsburgh and by the school board of the participating schools. An invitation letter that described the purpose and the procedure of the study was sent to the parents. A total of 950 invitation letters were sent out. Students who were 18 years old provided informed consent and students who were younger than 18 years of age provided assent after their parents provided informed consent. 


\subsubsection{Subjects:}

Ninety-one participants voluntarily enrolled from three local high schools in Allegheny County, PA, USA. Two of the schools were private parochial schools in an urban location (one female only, and one male only) and the other school was a public co-educational school in a suburban school district. The participants were excluded if they had a previous history of self-reported concussion or if they had a history of low back or lower extremity problems within three months prior to the date of testing. The demographic characteristics of participants (age in years, height, body mass) are summarized in Table 3-1. Participants were asked to report the duration of participation in formal athletic practice for their high school or club teams (Table 3-2).

Participants also reported the duration of their recreational physical activities.

Table 3-1: Demographic Characteristics of participants

\begin{tabular}{|l|c|c|}
\hline & Male & Female \\
\hline $\mathbf{n}$ & 47 & 44 \\
\hline Age in years, n & & 15 \\
\hline $\mathbf{1 4 ~ . 0}$ - 14.9 & 18 & 12 \\
\hline $\mathbf{1 5 . 0}-\mathbf{1 5 . 9}$ & 15 & 11 \\
\hline $\mathbf{1 6 ~ . 0}-\mathbf{1 6 . 9}$ & 5 & 6 \\
\hline $\mathbf{1 7 ~ . 0}-\mathbf{1 7 . 9}$ & 1 & 0 \\
\hline $\mathbf{1 8 . 0}-\mathbf{1 8 . 9}$ & $15.5(1.1)$ & $15.7(.9)$ \\
\hline Age in years, mean (SD) & $66(17)$ & $61(11)$ \\
\hline Mass in kg, mean (SD) & $172(10)$ & $166(6)$ \\
\hline Height in cm, mean (SD) & Yes: 34 & Yes: 31 \\
\hline Participation in formal sports & No: 13 & No: 13 \\
& &
\end{tabular}

n: Number of participants 
Table 3-2: Number of participants who participated in formal athletic activities

\begin{tabular}{|l|l|l|}
\hline \multicolumn{1}{|c|}{ Formal athletic activities } & Number of participants & Percent of total participants (\%) \\
\hline None & 26 & 28.6 \\
\hline Cross-country & 11 & 12.1 \\
\hline Basketball & 9 & 9.9 \\
\hline Soccer & 7 & 7.7 \\
\hline Volleyball & 7 & 7.7 \\
\hline Football & 5 & 5.5 \\
\hline Lacrosse & 5 & 5.5 \\
\hline Ice/ Field Hockey & 4 & 4.4 \\
\hline Crew & 4 & 4.4 \\
\hline Band & 3 & 3.3 \\
\hline Softball & 3 & 3.3 \\
\hline Baseball & 2 & 2.2 \\
\hline Weight lifting & 2 & 2.2 \\
\hline Gymnastics & 2 & 2.2 \\
\hline Biking & 2 & 2.2 \\
\hline Golf & 1 & 1.1 \\
\hline Bowling & 1 & 1.1 \\
\hline Wrestling & 1 & 1.1 \\
\hline Tennis & 1 & 1.1 \\
\hline
\end{tabular}

\subsubsection{Outcome measures}

The $\mathrm{ABC}$ is a sel f-report questionnaire which was used to assess the participant's level of confidence that they would not lose their balance while performing 16 functional activities. ${ }^{255}$

The highest possible score of 100 suggests maximum confidence and a score of 0 suggests no confidence.

The DGI is an eight item instrument that assessed the participants' ability to walk on a level surface, walk with head turns, walk with pivot turns, changes of speed, over and around obstacles and up and down steps. ${ }^{256}$ The scale for each item ranges from $0-3$, where 0 means 
severe impairment and 3 indicates normal performance. The highest possible score is 24 . Its concurrent validity has been established against the Berg Balance Scale in individuals with vestibular disorders. ${ }^{260}$ It has been reported that DGI scores less than 19 are correlated with reports of falls in community dwelling older adults and in individuals with vestibular disorders. $^{253,261-262}$

The FGA is a 10 -item test based on the DGI. ${ }^{263}$ The three new items introduced in the FGA were gait with a narrow base of support, gait with eyes closed, and ambulating backwards.

The maximum score is 30 . Higher scores indicate better performance. The FGA demonstrated concurrent validity with other measures used in vestibular rehabilitation $(r=.64$ to $.80) .{ }^{257}$

Gait speed was recorded while participants ambulated at their comfortable speed over 4 meters using a standing start and over $6.1 \mathrm{~m}$ using a walking start. The participant was instructed to "Walk to the other end of the course at your usual speed passing the marked line, just as if you are walking down the street to go to the store." The timing started when the first foot crossed the start line, and ended when the first foot crossed the stop line.

The TUG is a timed test during which the participant stands from a chair, walks three meters at their normal walking speed, returns to the chair and sits down. ${ }^{258}$ The participants were instructed as follows: "When I say start, I want you to stand and walk at your usual speed to the tape on the floor, and then come back and sit in the chair." The timing began when the examiner said "start" and ended when the participant's buttocks touched the chair. The TUG has been widely used in vestibular rehabilitation studies. ${ }^{264-267}$ It has been reported that slower TUG scores $(>11.5 \mathrm{sec})$ are correlated with reports of falls in individuals with vestibular disorders. ${ }^{253,268}$ 
The FTSTS test requires participants to stand-up and sit down from a chair (43 cm high), five times as quickly as possible. The participants completed the task with their hands crossed on their chest. ${ }^{259}$ The instructions were: "I want you to stand up and sit down 5 times as quickly as you can when I say "GO". Stand up fully during each time. Do not touch the back of the chair during each repetition. Keep your hands crossed on your chest." The timing period began when the examiner said "GO" and ended when the participant's buttocks touched the chair on the fifth repetition. The FTSTS test exhibited moderate correlation with gait and dynamic balance measures in patients with vestibular disorders. ${ }^{269}$ The discriminative and concurrent validity of the FTSTS test have been reported in patients with vestibular disorders. ${ }^{252}$

The Balance Error Scoring System (BESS) ${ }^{270}$ is a component of the Sport Concussion Assessment Tool 2, recommended by the Consensus Statement on Concussion in Sport. ${ }^{70}$ The BESS requires participants to maintain balance with eyes closed with their hands on their iliac crests under six different conditions: ${ }^{270}$ 1) firm surface, feet together, 2) firm surface, single leg stance, 3) firm surface, tandem stance, 4) foam surface, feet together, 5) foam surface, single leg stance, and 6) foam surface, tandem stance. Each trial was 20 seconds. $^{270}$ The scores were calculated by counting the total number of errors. Errors could be any of the following:1) hands lifted off the iliac crests, 2) opening eyes, 3) a step, stumble or fall, 4) moving the hip into >30 degrees of abduction or flexion, 5) lifting the forefoot or heel, or 6) remaining out of the test position for $>5$ seconds. The maximum number of errors in each trial was 10 and the maximum number of errors in total was 60. A systematic review has concluded that the BESS demonstrated moderate to good reliability and correlated well with other measures of balance using testing devices. ${ }^{271}$ 


\subsubsection{Procedure}

After the demographic and athletic participation information was recorded, participants completed the ABC. Participants then went to one of the three stations where the tests were performed: 1) GS, TUG, and FTSTS, 2) DGI/FGA, and 3) the BESS. Participants performed the testing in a pseudo- random order based on test station availability. Two consecutive trials of gait speed, TUG, and FTSTS were performed by the same examiner. In a sample of 61 participants who were examined by one of the raters, the two consecutive trials were used to calculate the test-retest reliability for the gait speed, TUG and FTSTS. Seven raters who were licensed physical therapists and routinely administered the tests participated in DGI/FGA scoring, and six physical therapy graduate students participated in the scoring of GS, the TUG and the FTSTS.

Reliability training was provided to all examiners before the testing started and the differences in timed tests between examiners was $0.1 \mathrm{sec}$. In a subset of the high school students $(n=23)$, an electronic timing device was used to record gait speed so that validity of the therapist administered gait speed could be determined.

The BESS performance was videotaped for later scoring using a cam era placed three meters from the participants, perpendicular to the frontal plane in quiet standing. The participant was facing the camera while performing the six different conditions. Inter- rater reliability was examined using two Doctor of Physical Therapy student raters who were provided with written instructions and trained to score the BESS. 


\subsubsection{Statistical analysis:}

Statistical analyses were conducted to determine if there were any associations between the subject demographic variables and the outcome measures. An independent t-test was performed to examine if there was an effect of gender or athletic participation on the normally distributed scores. Non-parametric Mann-Whitney $U$ test was used to examine the effect of gender or athletic participation on the non interval measures of ABC, DGI, FGA and the total BESS score.

Correlation analyses were performed to examine the relationship between age, height, body mass and the performance of gait and balance measures. Pearson correlation coefficients were calculated for normally distributed variables, and the Spearman-rho correlation coefficient was calculated for variables not normally distributed.

For the total score of each outcome measure, scores were calculated at the $5^{\text {th }}, 25^{\text {th }}, 50^{\text {th }}$, $75^{\text {th }}$, and $95^{\text {th }}$ percentiles. In addition, percentile scores were calculated for the individual items of the ABC, DGI and FGA. To determine reliability of GS, TUG and FTSTS, the intraclass correlation coefficient (ICC) was computed using the 2 way mixed model for the single measures $(3,1)$. From the ICC analysis, the $95 \%$ confidence interval, the standard error of measurement (SEM) and minimal detectable change (MDC) were calculated. The validity of clinician administered gait speed was tested against the electronic timing device by computing the ICC, using the two-way mixed model for single measures.

The inter-rater reliability of the total BESS score was examined by using the two-way mixed ICC model (random subjects, fixed raters, absolute agreement). Percent agreement, expected agreement and weighted kappa were calculated for the six individual conditions. 


\subsection{RESULTS}

Of the 108 participants who consented, 17 were excluded from the study due to previous history of concussion or a recent lower extremity injury. Ninety one participants participated in the study (47 M/44 F). The majority of the participants (65\%) were younger than 16 years old (Table 3-1).

Twenty- nine percent of the participants were not involved in any formal athletic participation (Table 3-2). For the participants who were involved in formal athletic participation, cross country running was the most common sport (11 participants). Three hours per week was the most common duration of formal practices ( 9 participants) followed by two hours per week (8 participants). However, the median was 7 hours per week (range: 1- 27 hours). An independent $\mathrm{t}$-test was performed to examine if the participants with no formal practice were significantly different in the performance on any of the administered measures. The results demonstrated that the group with no formal athletic performance had significantly worse scores on the FTSTS ( $\mathrm{M}=8.1, \mathrm{SD}=1.4 \mathrm{~s})$ compared with the group with formal athletic practice $(\mathrm{M}=$ $7.3, \mathrm{SD}=1.3 \mathrm{~s}),\left(\mathrm{t}_{89}=2.4, \mathrm{p}=.016\right)$.

There were no significant differences between males and females except for the $\mathrm{ABC}$ where male participants exhibited higher scores $(M=95, S D=5)$ compared to female $(M=92$, $\mathrm{SD}=7),(\mathrm{U}=762, \mathrm{p}=0.03)$. There was no significant correlation between height, weight and any of the administered measures. Age demonstrated a significant positive correlation with the TUG scores, $($ Spearman rho $=0.27, \mathrm{p}=.001)$. 


\subsubsection{Reference values}

The percentile reference scores for the ABC, DGI, FGA, TUG, GS (4m \& $6.1 \mathrm{~m}$ ) and FTSTS are detailed in Table 3-3. The distributions of timed performance tests of the TUG, GS and FTSTS were normal and did not exhibit ceiling or floor effect. Additionally, the percentile score for gait speed measured over a course of $4 \mathrm{~m}$ and $6.1 \mathrm{~m}$ revealed that walking speed was generally faster when tested over the course of $6.1 \mathrm{~m}$ (walking start). A ceiling effect was observed with the ABC, DGI, and FGA outcomes as demonstrated by the similarity of the scores from $50^{\text {th }}$ to $95^{\text {th }}$ percentile.

Table 3-3: Percentile scores for the outcome measures $(n=91)$

\begin{tabular}{|c|c|c|c|c|c|}
\hline & \multicolumn{5}{|c|}{ Percentile } \\
\hline $\begin{array}{c}\text { Activities - specific Balance } \\
\text { Confidence Scale (ABC) }\end{array}$ & $\mathbf{5}$ & $\mathbf{2 5}$ & $\mathbf{5 0}$ & $\mathbf{7 5}$ & $\mathbf{9 5}$ \\
\hline Dynamic Gait Index (DGI) & 22 & 91 & 95 & 98 & 99 \\
\hline $\begin{array}{c}\text { Functional Gait Assessment } \\
\text { (FGA) }\end{array}$ & 26 & 28 & 29 & 30 & 30 \\
\hline $\begin{array}{c}\text { Gait speed over 4m (m/sec) } \\
\text { Standing start }\end{array}$ & 0.84 & 1.09 & 1.19 & 1.29 & 1.46 \\
\hline $\begin{array}{c}\text { Gait speed over 6.1 m } \\
\text { (m/sec) }\end{array}$ & 1.03 & 1.23 & 1.32 & 1.44 & 1.69 \\
\hline Walking start & 6.1 & 6.9 & 7.5 & 8.3 & 9.3 \\
\hline Timed “UP \&GO" (sec) & 5.4 & 6.7 & 7.5 & 8.4 & 9.8 \\
\hline
\end{tabular}

Table 3-4 demonstrates the ceiling effect in more detail by listing scores for the individual items of the $\mathrm{ABC}$. A full confidence score of 100 was rated by $75 \%$ of the participants for 6 items, and by $50 \%$ for an additional 5 items. A closer inspection of the individual items of the $\mathrm{ABC}$ revealed that the question "Walk outside on icy sidewalks?" and "Step onto or off an escalator while you are holding onto parcels such that you cannot hold onto the railing?" were the two items on the $\mathrm{ABC}$ scale in which subjects had the least confidence in performing. The 
DGI also demonstrated a ceiling effect in which the full score (24/24) was achieved by more than 50 percent of the participants. To a lesser extent, the FGA also exhibited a ceiling effect in which the optimal score was achieved by at least $25 \%$ of the participants. The FGA percentile scores revealed that gait with eyes closed was the most difficult item. A score of 3 (full score) was achieved for all other items by $75 \%$ of the participants (Table 3-5).

Table 3-4: Percentile scores for individual items on the Activities-specific Balance Confidence (ABC) Scale $(\mathbf{n}=91)$

\begin{tabular}{|c|c|c|c|c|c|}
\hline & \multicolumn{5}{|c|}{ Percentile } \\
\hline & 5 & 25 & 50 & 75 & 95 \\
\hline Walk around the house? & 90 & 100 & 100 & 100 & 100 \\
\hline Walk up and down the stairs? & 80 & 90 & 100 & 100 & 100 \\
\hline $\begin{array}{l}\text { Bend over and pick up a slipper } \\
\text { from in front of a closet door? }\end{array}$ & 80 & 90 & 100 & 100 & 100 \\
\hline $\begin{array}{l}\text { Reach for a small can off a shelf at } \\
\text { eye level? }\end{array}$ & 90 & 100 & 100 & 100 & 100 \\
\hline $\begin{array}{l}\text { Stand up on } t \text { ip toes and reach for } \\
\text { something above your head? }\end{array}$ & 70 & 80 & 90 & 100 & 100 \\
\hline $\begin{array}{l}\text { Stand on a chair and reach for } \\
\text { something? }\end{array}$ & 70 & 80 & 90 & 100 & 100 \\
\hline Sweep the floor? & 80 & 100 & 100 & 100 & 100 \\
\hline $\begin{array}{l}\text { Walk outside the house to a car } \\
\text { parked in the driveway? }\end{array}$ & 90 & 100 & 100 & 100 & 100 \\
\hline Get into or out of a car? & 80 & 90 & 100 & 100 & 100 \\
\hline $\begin{array}{l}\text { Walk across the parking lot to a } \\
\text { mall? }\end{array}$ & 90 & 100 & 100 & 100 & 100 \\
\hline Walk up or down a ramp? & 80 & 100 & 100 & 100 & 100 \\
\hline $\begin{array}{l}\text { Walk in a crowed mall where people } \\
\text { rapidly walk past you? }\end{array}$ & 70 & 90 & 100 & 100 & 100 \\
\hline $\begin{array}{l}\text { Are bumped into by p eople as you } \\
\text { walk through the mall? }\end{array}$ & 60 & 80 & 90 & 100 & 100 \\
\hline $\begin{array}{l}\text { Step onto or off of an escalator } \\
\text { while you are holding onto a railing? }\end{array}$ & 70 & 90 & 100 & 100 & 100 \\
\hline $\begin{array}{l}\text { Step onto or off an escalator while } \\
\text { you are holding onto parcels such } \\
\text { that you cannot hold onto the } \\
\text { railing? }\end{array}$ & 50 & 80 & 90 & 100 & 100 \\
\hline Walk outside on icy sidewalks? & 40 & 70 & 80 & 90 & 100 \\
\hline
\end{tabular}


Table 3-5: Percentile scores for individual items on the Functional Gait Assessment (FGA)

\begin{tabular}{|c|c|c|c|c|c|}
\hline & \multicolumn{5}{|c|}{ Percentile } \\
\hline & 5 & 25 & 50 & 75 & 95 \\
\hline Gait Level Surface & 2 & 3 & 3 & 3 & 3 \\
\hline Change In Gait Speed & 2 & 3 & 3 & 3 & 3 \\
\hline $\begin{array}{l}\text { Gait With Horizontal Head } \\
\text { Turns }\end{array}$ & 2 & 3 & 3 & 3 & 3 \\
\hline Gait With Vertical Head Turns & 3 & 3 & 3 & 3 & 3 \\
\hline Gait And Pivot Turn & 3 & 3 & 3 & 3 & 3 \\
\hline Step Over Obstacle & 2 & 3 & 3 & 3 & 3 \\
\hline $\begin{array}{l}\text { Gait With Narrow Base Of } \\
\text { Support }\end{array}$ & 2 & 3 & 3 & 3 & 3 \\
\hline Gait With Eyes Closed & 0 & 2 & 3 & 3 & 3 \\
\hline Ambulating Backwards & 2 & 3 & 3 & 3 & 3 \\
\hline Stair Climbing & 2 & 3 & 3 & 3 & 3 \\
\hline
\end{tabular}

The BESS test demonstrated variability in scores depending on the six conditions (Table 3-6). The condition of single leg stance on foam surface was the most difficult condition, followed by the conditions of single leg stance on a flat surface and tandem stance on a foam surface where these two items were almost equally difficult. Stance with feet together exhibited a ceiling effect in which the majority of participants did not commit any errors except a few participants who had one error while the task was performed on the foam surface. 
Table 3-6: Percentile scores for the Balance Error Scoring system (BESS). Lower scores indicate "better" performance on the BESS

\begin{tabular}{|l|c|c|c|c|c|}
\hline & \multicolumn{5}{|c|}{ Percentile } \\
\hline & $\mathbf{5}$ & $\mathbf{2 5}$ & $\mathbf{5 0}$ & $\mathbf{7 5}$ & $\mathbf{9 5}$ \\
\hline Firm surface, feet together & 0 & 0 & 0 & 0 & 0 \\
\hline Firm surface, single leg stance & 0 & 1 & 2 & 4 & 7 \\
\hline Firm surface, tandem & 0 & 0 & 0 & 1 & 4 \\
\hline Foam surface, feet together & 0 & 0 & 0 & 0 & 1 \\
\hline Foam surface, single leg stance & 2 & 5 & 7 & 10 & 10 \\
\hline Foam surface, tandem & 0 & 1 & 3 & 4 & 7 \\
\hline Total BESS scores & $\mathbf{4}$ & $\mathbf{1 0}$ & $\mathbf{1 3}$ & $\mathbf{1 8}$ & $\mathbf{2 4}$ \\
\hline
\end{tabular}

\subsubsection{Reliability analysis}

The test retest reliability for the gait speed, TUG and FTSTS ( $n=61 q)$ with $95 \%$ confidence interval, SEM and MDC are summarized in Table 3-7. Gait speed measured over the course of 6.1 m using a walking start exhibited similar values for reliability coefficient, SEM and MDC compared to gait speed measured over a course of $4 \mathrm{~m}$ using a standing start. The ICC for clinician administered gait speed vs. electronic timing was 0.98 with 95\% CI (0.95 - 0.99).

Table 3-7: Reliability coefficients, standard error of the measurement (SEM), and minimal detectable change (MDC) for the timed measures $(n=61)$

\begin{tabular}{|l|c|c|c|}
\hline & ICC (95 \%CI) & SEM & MDC \\
\hline Gait speed (4 m) & $0.81(0.71-0.88)$ & $0.07 \mathrm{~m} / \mathrm{sec}$ & $0.18 \mathrm{~m} / \mathrm{sec}$ \\
\hline Gait speed (6.1 m) & $0.79(0.67-0.87)$ & $0.07 \mathrm{~m} / \mathrm{sec}$ & $0.21 \mathrm{~m} / \mathrm{sec}$ \\
\hline Timed “UP\&GO” & $0.84(0.75-0.90)$ & $0.3 \mathrm{sec}$ & $0.9 \mathrm{sec}$ \\
\hline Five Times Sit to Stand & $0.91(0.86-0.95)$ & $0.1 \mathrm{sec}$ & $0.4 \mathrm{sec}$ \\
\hline
\end{tabular}


The percent agreement and weighted kappa statistics for the BESS are summarized in Table 3-8. The inter-rater reliability for the total score was 0.95 . Weighted kappa statistics cannot be calculated for the condition with feet together on a firm surface due to the lack of variability between raters. For the other five conditions, the weighted kappa scores ranged between 0.46 and 0.79 .

Table 3-8: Percent agreement, expected agreement, and weighted kappa statistics for the individual conditions of the Balance Error Scoring System (BESS).

\begin{tabular}{|l|c|c|c|c|}
\hline BESS Condition & Agreement & $\begin{array}{c}\text { Expected } \\
\text { agreement }\end{array}$ & kappa & p-value \\
\hline Firm surface, feet together & $\dagger$ & $\dagger$ & $\dagger$ & $\dagger$ \\
\hline Firm surface, single leg stance & $94 \%$ & $72 \%$ & 0.77 & $<0.01$ \\
\hline Firm surface, tandem & $95 \%$ & $77 \%$ & 0.79 & $<0.01$ \\
\hline Foam surface, feet together & $96 \%$ & $93 \%$ & 0.46 & $<0.01$ \\
\hline Foam surface, single leg stance & $91 \%$ & $72 \%$ & 0.68 & $<0.01$ \\
\hline Foam surface, tandem & $92 \%$ & $70 \%$ & 0.72 & $<0.01$ \\
\hline
\end{tabular}

$\dagger$ kappa cannot be calculated because there was no variability in the scores.

\subsection{DISCUSSION}

The results demonstrated that GS, TUG, and FTSTS are reliable and can be used for adolescents with gait and balance deficits after concussion. Although the ABC, DGI and FGA exhibited a ceiling effect in this healthy young population, the distributions of the timed tests of GS (4 m \& $6.1 \mathrm{~m})$, TUG, and FTSTS did not exhibit a ceiling or floor effect, and therefore we believe they can be useful in tracking the recovery of gait and balance deficits in individuals between age of 
14 and 18. In addition, the inter-rater reliability of the BESS is excellent when performance is video-recorded and scored at a later time.

\subsubsection{Reference values}

Review of the normative scores revealed that the ABC, DGI and FGA had a ceiling effect, which may limit their usefulness in this population. A potential alternative to using the full $\mathrm{ABC}$ is to use the recently evaluated $\mathrm{ABC}-6$ questionnaire, an abbreviated version of the full $\mathrm{ABC}$ that includes item numbers $5,6,13,14,15$, a nd 16 and is suggested to have psychometric properties analogous to the full $\mathrm{ABC}$ questionnaire. ${ }^{272} \mathrm{~A}$ closer look at the individual items of the $\mathrm{ABC}$ in the current study revealed that the items included in the ABC- $6^{272}$ exhibited lower scores and had more variation than other items in the full version of $\mathrm{ABC}$. However, a validation study is needed before a recommendation is made to adopt the use of an abbreviated version over the full version of $\mathrm{ABC}$.

The performance measures of the DGI and FGA also exhibited a ceiling effect, suggesting that these tests do not assess higher level balance abilities that children in this age range may possess. Therefore, a return to "normal" on these tests may not indicate that they have fully achieved their pre-morbid balance capability. Since the FGA has less of a ceiling effect compared to DGI in this age group, the use of the FGA is recommended over the DGI. These findings emphasize the importance of using comprehensive assessment measures; this conservative approach is in line with the recent recommendations on concussion management

endorsed by the National Athletic Trainers Association and the $3^{\text {rd }}$ International Conference on Concussion in Sport. $^{70,84}$ 
To the best of our knowledge, there are no age-specific normative reference scores available for the DGI. One study provided normative reference values for the FGA; ${ }^{273}$ the study included community dwelling older adults between the ages of 40 to 89 years and the normative reference values for each decade. The study did not include participants younger than 40 years based on the assumption that FGA performance is not expected to decline with age until middle age or later. The mean score and range obtained for the participants between 40 and 49 years was 29 (range 24-30) which matches the median score and range obtained in our study. ${ }^{273}$ The item of ambulating backward was reported to be the most difficult item in this study and in the study of Walker et al. ${ }^{273}$ A comparison of the performance on individual items revealed that a full score of 3 was achieved by more than $50 \%$ of our participants for all items. However, participants between 40 and 49 years old exhibited average scores that were less than 3 on the items of gait on level surface, gait with eyes closed, and ambulating backwards.

Examination of the normative values showed that gait speed was faster when measured over $6.1 \mathrm{~m}$ compared with $4 \mathrm{~m}$, which can probably be explained by the difference in the initiation of movement.

Over the course of $4 \mathrm{~m}$, the participants initiated walking at the start line. However, over the $6.1 \mathrm{~m}$ course, walking was initiated one meter before the starting line. This difference in walking speed between the two methods suggests that clinicians should be consistent with their method of administration of gait speed and use the appropriate norms. A previous study established normative scores for participants that were similar to our age range. ${ }^{274}$ However, the previous normative reference values were provided separately for male and female participants.

This is in contrary to our findings that there was no gender effect on gait speed in this age range. Nonetheless, the median gait speed of the $6.1 \mathrm{~m}$ reported in our study $(1.32 \mathrm{~m} / \mathrm{sec})$ is 
consistent with the means provided for males $(1.35 \mathrm{~m} / \mathrm{sec})$ and females $(1.24 \mathrm{~m} / \mathrm{sec})$ between 15 and 19 years old. ${ }^{274}$

The positive correlation between age and the TUG scores was unexpected and may be related to the casual walking pattern exhibited by many of the older students, or the limited number of older participants. The difference in the FTSTS scores between participants who were involved in formal athletic practice and those who are not involved in athletic practice was also unexpected. The participants were instructed to perform the task as quickly as possible; therefore, participants with formal sports practices may have demonstrated better functional lower extremity strength, which is known to affect the repetitive sit to stand performance, compared to participants with no formal athletic involvement. ${ }^{275}$ Although exploring the differences in FTSTS scores between different sports would be of interest, this goal was unattainable with the limited number of participants in each sport category. Overall, the absence of a relationship between age, gender, height and body mass to most of the administered measures suggests the normative reference values provided in this manuscript can be used for all individuals in this age range.

The distribution of total BESS scores appear to be similar to several previous studies of high school and college-age subjects that have examined BESS performance in the context of differentiating athletes with and without concussion and also for different sports. ${ }^{31,144,173,276-278}$

The median number of errors accumulated in this age group (13 errors) compares well to the average number of errors accumulated in high school and collegiate athletes without history of concussion (range 12 to 13 errors). ${ }^{31,173,270,277-278}$ One benefit of the current study is that it also provides percentile values for each of the individual conditions. Compared with previous studies of high school aged subjects, ${ }^{277,279}$ the median values of the individual conditions are 
similar to the average values of the previous studies, except for the single leg stance on foam condition, which had a higher number of errors in the current study (7 vs. 4 to 5). Differences in reference values of the BESS across the different studies can be attributed to several factors, including inherent differences in balance performance across different age ranges and sport abilities, disparate scoring methods (i.e. direct observation vs. Videotaping), rater experience, and participant learning effects. Regarding balance abilities of different sports, female gymnasts accumulated an average of 9.1 errors, female collegiate soccer players had 12.5 errors, and female basketball athletes accumulated 14.1 errors. ${ }^{276}$ Clinicians who intend to use these scores are encouraged to consider the limitations of these reference values and are encouraged to consider the degree to which their patients match the participants included in this study.

\subsubsection{Reliability analysis:}

The reliability for the timed measures examined in this study (GS, TUG, and FTSTS) ranged between 0.79 and 0.91 , suggesting that these measures have moderate to good reliability and can be used during vestibular rehabilitation for this age group. For the FTSTS, the ICC score reported in the present study [0.91 (95\% CI $0.86-0.95)]$ falls in the wide range of reliability coefficients previously reported in other populations $[0.64-0.99] .{ }^{259,280-284}$ Test-retest reliability of TUG and GS were lower in this group of adolescents than what has been published for older adults. Reliability of the TUG generally is greater than $0.9,{ }^{258,285}$ although the reliability has

been reported as low as $0.5{ }^{286}$ Similarly, reliability of gait speed has been reported at 0.9 and above. $^{285,} 287$ It is unclear why adolescents have lower test-retest reliability for walking speed demonstrated by the GS and TUG measures. 
The inter-rater reliability ICC for the total BESS score was 0.95 . This score is higher than inter-rater reliability scores previously reported. McLeod reported an inter-rater ICC score of 0.85 in a sample of female high school basketball players. ${ }^{277}$ While the inter-rater reliability scores for individual conditions have ranged from 0.78 to $0.96 .{ }^{270}$ However, Finnoff et al. has reported an ICC score of 0.57 in a sample of 30 college athletes. ${ }^{288}$ Several reasons may have contributed to the differences in inter-rater reliability reported among the studies. Our sample size of 91 participants was much higher than the other two studies, which may suggest that the previous studies were underpowered; in McLeod et al, ${ }^{277}$ although the overall sample consisted of 62 participants, the analysis of inter- rater reliability was conducted only for a subset of this sample and there were no $f$ urther demographics details provided for the reliability subset provided. ${ }^{277}$ The other study by Finnoff et al included a sample size of 30 participants that were rated by three raters. ${ }^{288}$ The balance abilities of the subjects may also have played a role. Our participants were different than participants reported in other studies as in Finoff et al's study where participants were described as "athletes" with no further description, while McLeod et al's study consisted of female basketball players. ${ }^{277}$ However, our study included athletic and nonathletic male and female participants. This may have led to a larger variability in scores and improved the ICC value. Additionally, the raters in our study scored the BESS performance by viewing a videotape; this is contrary to McLeod et al. in which the BESS was scored by direct observation. ${ }^{277}$ Although Finnoff et al have used videotapes for scoring, ${ }^{288}$ their ICC score was much lower than ours; it is unclear if the self-selected pace of viewing the videotapes used in our study improved the reliability, whereas in the Finnoff et al's study the examiners were asked to score 30 participants consecutively in the same session and therefore, fatigue may have resulted in less accurate scoring. ${ }^{289}$ 


\subsection{LIMITATIONS}

Due to recruitment difficulties for students in the upper grades of high school, the majority of our participants were $14 \& 15$ years old. However, age was not found to have an effect on the balance tests (except TUG) and therefore, the normative scores presented in the study can be used for all high school age students. In addition, the participants in this study were students from private and suburban schools in one geographic area. They may represent certain socioeconomic status (SES) and may not reflect the wide spectrum of high school aged children.

\subsection{CONCLUSION}

These normative scores provide a $\mathrm{r}$ eference for interpreting the performance of common functional gait and balance measures for individuals between 14 and 18 years of age who require vestibular rehabilitation, such as individuals who sustain concussion. These normative scores may provide end points for discharge from the vestibular physical therapy. Age did not affect the performance of the administered measures (except TUG). Therefore, the normative scores presented in the study can be used for all high school age students. The inclusion of non athletic participants makes the normative scores presented in this manuscript applicable for both athletes and non athletes who might be susceptible to mild TBI. 


\subsection{SECOND AIM}

\subsection{INTRODUCTION}

Concussion is one of the most prevalent acquired neurological conditions occurring in children and young a dults. ${ }^{8,} 186$ According to the Centers for Disease Control (CDC), concussion is synonymous with the term "mild TBI". ${ }^{186}$ Using the definition proposed by $t$ he CDC, ${ }^{186}$ concussion is "a complex pathophysiologic process affecting the brain, induced by traumatic biomechanical forces secondary to direct or indirect forces to the head. It is caused by a blow or jolt to the head that disrupts the function of the brain. This disturbance of brain function is typically associated with normal structural neuroimaging findings (i.e., CT scan, MRI). It results in a constellation of physical, cognitive, emotional and/or sleep-related symptoms and may or may not involve a loss of consciousness (LOC)." Duration of symptoms is highly variable and may last from several minutes to days, weeks, months, or even longer in some cases. Some factors which may contribute to prolonged recovery include loss of consciousness, amnesia, and confusion; ${ }^{290}$ however, our understanding of this issue is still very limited.

Dizziness is a frequent symptom of concussion and has been reported in range from $23 \%$ - $81 \%$ of cases within the first days post-injury. Estimates of the prevalence of persistent dizziness after mTBI varies widely from $1.2 \%$ at 6 months to $32.5 \%$ at five years ${ }^{183,}{ }^{291-293}$ Poor 
balance and postural instability have been reported in many studies post- concussion, ${ }^{13,48,294}$ and have been correlated with dysfunction in sensory integration. ${ }^{146,295}$

Despite the high incidence of dizziness and balance dysfunction in people who have had a concussion, reports of vestibular and balance rehabilitation in the management of concussion are sparse. ${ }^{34,44,234-235}$ Part of the reason for the lack of information is that in many cases, symptoms resolve relatively quickly before referrals to tertiary providers can be made. In one study, over $75 \%$ of high school football players returned to play within 3 weeks of their concussion. $^{296}$ The use of vestibular rehabilitation in the treatment of concussion-related dizziness and balance dysfunction has been promising, although Shepard et al. ${ }^{235}$ commented that the duration of vestibular rehabilitation is longer in people with head injury compared with unilateral peripheral vestibular dysfunction.

It has not been established if the effects of concussion regarding dizziness and balance dysfunction are the same for children and adults. It is also unclear whether the amount of recovery form dizziness and balance dysfunction is different between children and adults after concussion. ${ }^{4,242-243}$ Children's tolerance to biochemical changes associated with concussion may be different from adults, and consequently the sequelae from impacts of the same magnitude may be different between children and adults. ${ }^{4}$ Furthermore, the role that it is played by the continuous and rapid maturation in children's cognitive abilities and postural strategies in the recovery process is unknown., 240, 242, 297

The purpose of this retrospective study was to describe the severity of dizziness symptoms and balance dysfunction reported by people who were referred for vestibular rehabilitation after concussion. Furthermore, this study described the outcomes of vestibular 
rehabilitation on reducing dizziness and balance dysfunction, and described if the amount of recovery during vestibular rehabilitation was different between adults and children.

\subsection{METHODS}

\subsubsection{Participants}

A retrospective case series was performed of 114 consecutive participants, including children, who were referred between 2006 a nd 2008 to a tertiary balance center for vestibular rehabilitation after being diagnosed with a concussion. For the purpose of this study, children were defined as 18 years and younger, and the adults were defined as older than 18 years. The median age for children ( 45 female, 22 male) was $16 \mathrm{y}$ with a range of 8 to $18 \mathrm{y}$, and the median age for the adults ( 25 female, 22 male) was $41 \mathrm{y}$ with a range of 19 to $73 \mathrm{y}$. Of the 114 participants who were examined, 84 had more than one visit and 30 had a single visit. The study was approved by Institutional Review Board (IRB) at the University of Pittsburgh.

\subsubsection{Intervention and outcome measures}

The vestibular rehabilitation intervention consisted of a customized program that was tailored to each subject's impairments and functional limitations that related to dizziness, ocular motor

function, and balance function. ${ }^{298}$ The categories of exercises most frequently provided in treatment and in the home exercise program included gaze stabilization exercises (e.g. VOR x1 in sitting and standing), standing balance (e.g. standing with feet apart, feet together, on foam, 
with eyes open and closed), ambulation (e.g. walking with head turns, tandem walking, obstacle avoidance) and in a few cases, canalith repositioning manuevers. Exercises were prescribed to be done daily.

Self-report and performance measures were administered at the initial evaluation as well as at weekly and monthly intervals. The time points considered for this report include the initial evaluation and discharge scores. If a measure was not recorded at the time of initial evaluation or discharge, the assessment at the time point closest to the time of initial evaluation or discharge was used.

\subsubsection{Self-report measures}

Participants were asked to rate their current dizziness severity on a verbal scale from $0-100$, where 0 means no dizziness and 100 means maximum dizziness. Verbal anchors relating to severity of dizziness (e.g. slight, mild, moderate, severe) were provided for the scale. In addition, participants were asked to describe their dizziness using any of the following non-exclusive terms: spinning, lightheadedness, off balance, nausea, sensation of motion, and other.

The Activities-specific Balance Confidence scale $(\mathrm{ABC})$ is a questionnaire used to assess the respondent's level of confidence that they would not lose their balance while performing 16 functional activities. The highest possible score of 100 suggests maximum confidence and a score of 0 suggests no confidence. ${ }^{255}$

The Dizziness Handicap Inventory (DHI) is an instrument used to assess the individual's handicap due to their dizziness in 25 items relating to physical, emotional, and functional domains. The highest overall score on the test is 100 and higher scores indicate greater handicap resulting from dizziness. ${ }^{299}$ 


\subsubsection{Gait and balance Performance measures}

The Dynamic Gait Index (DGI) is an eight item instrument that assesses the ability to walk with head turns, changes of speed, and around obstacles. The scale for each item ranges from 0-3, where 0 means severe impairment and 3 means normal. The highest possible score is $24 .^{256}$

The Functional Gait Assessment (FGA) is a 10-item test based on the DGI. The maximum score is 30 . Higher scores indicate better performance. ${ }^{263}$

While participants were asked to walk at their comfortable speed, gait speed was timed over 4 meters course using stopwatch.

The Timed "Up\& Go" (TUG) is a timed test during which the participant stands from a chair, walks three meters at their normal walking speed, and returns to the chair. ${ }^{258}$

The Five Times Sit To Stand (FTSTS) requires participants to stand-up and sit down from a standard height chair five times as quickly as possible. The participants were asked to complete the task with their hands crossed on their chest. ${ }^{259}$

Dynamic Computerized Posturography: Participants performed the Sensory Organization Test (SOT, Neurocom, Inc.) under six different sensory conditions:1) eyes open, fixed support; 2)eyes closed, fixed support; 3)sway-referenced vision, fixed support; 4) eyes open, swayreferenced support; 5) eyes closed, sway-referenced support; 6) sway-referenced vision and support surface. Three $20 \mathrm{~s}$ trials were performed for each condition. The highest theoretical equilibrium score is 100 which indicates no sway; losses of balance were graded as zero.

Average scores for each condition were recorded, and the composite score was calculated using a weighted average of the individual trials. 


\subsubsection{Statistical analysis}

The process of care including time between the concussion and initial evaluation for vestibular rehabilitation, number of visits, and duration of treatment is summarized by descriptive statistics.

\subsubsection{Process of care}

The non-parametric Mann-Whitney $U$ test was performed to examine if there was a significant difference between participants who continued after initial evaluation and those who did not for the time between concussion onset and initial evaluation for vestibular rehabilitation therapy.

The Mann-Whitney U test was also performed to examine if there a significant difference in treatment duration, and number of visits between children and adults who received vestibular rehabilitation therapy.

\subsubsection{Outcome measures}

Independent t-tests were performed to determine if there was a difference in outcome measures at initial evaluation between participants who were referred to vestibular rehabilitation therapy but did not continue after their initial evaluation, and those who continued their intervention.

Independent t-tests also were performed to see if there was a difference in outcome measures between children and adults at the time of initial evaluation. For the independent t-test, Levine's test of equality of variance was examined. In those outcome measures that did not have equal variance, the p-value from the test that did not assume equal variances was used.

For the participants who had at least 2 visits, a mixed-factor repeated measures analysis of variance was performed on each outcome measure to see if there was an effect of time, age group, and interaction (time*age). The within-subjects factor was time with two levels (pre- 
treatment and post-treatment). The between-subjects factor was the age group with two levels (children 18 years and younger, and adults). For each outcome measure, only the participants who had data at both time points were included in the repeated measures ANOVA, and the sample size is reported for each. For all analyses, the level of significance was $\alpha=0.05$.

\subsection{RESULTS}

The vestibular rehabilitation was provided by 8 physical therapists; two of the therapists treated approximately $44 \%$ and $27 \%$ of the cases. The most frequent description of dizziness was a complaint of being "off balance" in $68 \%$ of the participants, followed by "lightheadedness" (54\%), "spinning" (46\%), "nausea" (38\%) and "sensation of motion" (23\%). "Off balance" was the most frequent description in both children and adults. Five participants had BPPV. One of participants with BPPV did not return after his initial visit. Three participants had pure BPPV, and a canalith repositioning maneuver was used as the only intervention. Finally, the fifth participant with BPPV also had other dizziness and balance dysfunction that was treated with a customized program in addition to the canalith repositioning maneuver.

\subsubsection{Process of care}

Of the 114 participants, 30 received an initial evaluation without returning for a second visit.

Reasons for not returning included: physical therapy was not indicated $(n=6)$, the participant lived far away $(n=8)$, and noncompliance $(n=16)$. The 2 groups of participants who came only for initial evaluation and those who returned for more visits did not differ based on 
age $\left(\mathrm{t}_{112}=1.1, \mathrm{p}=0.31\right)$, gender $\left(\chi^{2}=2.6, \mathrm{p}=0.11\right)$, or duration of time between the concussion and referral to the vestibular rehabilitation clinic (median 61, range 6 - 2566, MannWhitney $U=717, \mathrm{p}=.07$, Table 4-1).

Eighty-four (84) participants returned for at least 1 additional visit, and the median number of visits was 4 (range 2-13), occurring over a median duration of 33 (range 7-181) days.

There was no significant difference between children and adults in the number of visits (Mann- Whitney $U=730, p=.323$ ) or treatment duration (Mann- Whitney $U=723, p=.363$ ).

\subsubsection{Outcome measures at initial evaluation}

Outcome measures obtained during the initial evaluation for all 114 participants are reported in Table 4-1. Participants who did not continue after initial evaluation had significantly better scores on the dizziness severity, ABC, DHI, DGI, FGA, Gait Speed, and FTSTS $(\mathrm{p}<0.05)$. The FTSTS was the only outcome measure that was significantly different between the children and the adults at the time of initial evaluation, with children having faster performance (children 9.7 $\pm 3.5 \mathrm{~s}$, adults $13.2 \pm 5.4 \mathrm{~s}, \mathrm{t} 49.8=-3.3, \mathrm{p}=.002)$. 


\subsubsection{Change in outcome measures}

For participants who had received vestibular rehabilitation therapy, there was a significant time effect for all of the self-report and performance measures (Table 4-2).

Table 4-3 displays the outcome measures in which there was a significant interaction between time and age. There was a significant interaction between time and age for dizziness severity $\left(\mathrm{F}_{1,62}=8.6, \mathrm{p}=.005\right)$. Post hoc analysis revealed that after treatment, children's dizziness severity was reduced by mean of 19 points $\left(\mathrm{F}_{1,40}=31.0, \mathrm{p}<.001\right)$. However, there was no significant difference in dizziness severity for the adult group between pre-treatment and post-treatment time points $\left(\mathrm{F}_{1,22}=.06, \mathrm{p}=.805\right)$ (Table 4-3). There were also significant interactions between time and age for Conditions 1 and 2 of the $\operatorname{SOT}\left(\mathrm{F}_{1,19}=6.7, \mathrm{p}=.018\right.$ and $\mathrm{F}$ $1,19=5.9, \mathrm{p}=.025 \mathrm{r}$ espectively, Table 4-3). In both conditions, children had significant improvement in their scores $(\mathrm{p}<.01)$. However, there was no significant improvement for adults.

Only three of the measures demonstrated a significant age effect collapsing across time.

Children demonstrated significantly lower (better) scores in DHI $(36 \pm 6)$ compared with adults $(46 \pm 20)\left(\mathrm{F}_{1,67}=5.8, \mathrm{p}=.019\right)$. Children showed significantly higher scores on the FGA $(25 \pm 2)$ compared with adults $(23 \pm 3)\left(\mathrm{F}_{1,46}=5.0, \mathrm{p}=.030\right)$. Children also had lower FTSTS $(9.5 \pm 2.6 \mathrm{~s})$ compared with adults $(13.8 \pm 5.8 \mathrm{~s})\left(\mathrm{F}_{1,34}=8.7, \mathrm{P}=.006\right)$ 
Table 4-1: Mean (SD) of demographic and outcome measures at time of initial evaluation for vestibular rehabilitation, according to those participants who had evaluation only, and those who returned for a least 1 additional visit

\begin{tabular}{|c|c|c|c|}
\hline Outcome measure & $\begin{array}{l}\text { Evaluation only } \\
\qquad(\mathrm{n}=30)\end{array}$ & $\begin{array}{l}\text { Intervention } \\
(\mathrm{n}=84)\end{array}$ & Statistic, $\mathrm{p}$-value $\dagger$ \\
\hline Gender & $15 \mathrm{~F}, 15 \mathrm{M}$ & $56 \mathrm{~F}, 28 \mathrm{M}$ & $\chi^{2}=2.6, \mathrm{p}=0.106$ \\
\hline Age (y) & $28(16)$ & $25(15)$ & $\mathrm{t}_{112}=1.1, \mathrm{p}=0.314$ \\
\hline $\begin{array}{l}\text { Median (range) days from concussion to } \\
\text { evaluation }\end{array}$ & $96(8-2566)$ & $58(6-1149)$ & $\mathrm{U}=717, \mathrm{p}=0.07$ \\
\hline $\begin{array}{l}\text { Dizziness Severity } \\
\text { ( } 63 \text { children, } 46 \text { adults })\end{array}$ & $11(20)$ & $23(21)$ & $\mathrm{t}_{52.1}=-2.8, \mathrm{p}=.008^{*}$ \\
\hline ABC (65 children, 46 adults) & $78(25)$ & $65(28)$ & $\mathrm{t}_{109}=2.2, \mathrm{p}=.028^{*}$ \\
\hline DHI (66 children, 46 adults) & $37(19)$ & $48(22)$ & $\mathrm{t}_{110}=-2.5, \mathrm{p}=.014^{*}$ \\
\hline DGI (60 children, 36 adults) & $22(3)$ & $21(3)$ & $\mathrm{t}_{94}=2.3, \mathrm{p}=.027 *$ \\
\hline FGA (60 children, 36 adults) & $27(5)$ & $24(5)$ & $\mathrm{t}_{94}=2.5, \mathrm{p}=.013^{*}$ \\
\hline Gait Speed (61 children, 38 adults) & $1.21(.23)$ & $1.07(.26)$ & $\mathrm{t}_{97}=2.2, \mathrm{p}=.033^{*}$ \\
\hline TUG (s) (51 children, 32 adults) & $7.9(1.5)$ & $9.0(2.3)$ & $\mathrm{t}_{81}=-1.8, \mathrm{p}=.070$ \\
\hline FTSTS (s) (50 children, 33 adults) & $7.9(2)$ & $11.7(5)$ & $\mathrm{t}_{47.4}=-4.8, \mathrm{p}<.001^{*}$ \\
\hline SOT(Composite) (38 children, 21 adults) & $70(12)$ & $55(20)$ & $\mathrm{t}_{57}=1.7, \mathrm{p}=.083$ \\
\hline SOT Condition1 (38 children, 20 adults) & $83(16)$ & $88(11)$ & $\mathrm{t}_{5.5}=-1.1, \mathrm{p}=.482$ \\
\hline SOT Condition 2 (38 children, 20 adults) & $85(12)$ & $80(15)$ & $\mathrm{t}_{56}=.71, \mathrm{p}=.478$ \\
\hline SOT Condition 3 (38 children, 20 adults) & $86(9)$ & $75(22)$ & $\mathrm{t}_{56}=1.2, \mathrm{p}=.251$ \\
\hline SOT Condition 4 (38 children, 20 adults) & $74(9)$ & $53(26)$ & $\mathrm{t}_{18}=4.1, \mathrm{p}<.001^{*}$ \\
\hline SOT Condition 5 (38 children, 20 adults) & $52(15)$ & $37(25)$ & $\mathrm{t}_{8.7}=2.2, \mathrm{p}=.057$ \\
\hline SOT Condition 6 (38 children, 20 adults) & $59(18)$ & $41(24)$ & $\mathrm{t}_{56}=1.8, \mathrm{p}=.074$ \\
\hline
\end{tabular}

$\mathrm{SD}$, standard deviation, M, male; F, female; $\mathrm{ABC}=$ Activities-specific Balance Confidence scale, DHI = Dizziness Handicap Inventory, DGI = Dynamic Gait Index, FGA = Functional Gait Assessment, TUG = Timed "Up \& Go", FTSTS $=$ Five Times Sit To Stand, SOT $=$ Sensory Organization Test, $\uparrow \mathrm{t}$ is the Independent sample $\mathrm{t}$-test, $\mathrm{U}$ is the Mann-Whitney U test, $* \mathrm{p}<.05$ 
Table 4-2: Mean (SD) of outcome measures at times of initial evaluation and discharge

\begin{tabular}{|c|c|c|c|}
\hline Outcome Measure & Pre-treatment & Post-treatment & F-test \& p-value \\
\hline $\begin{array}{l}\text { Dizziness Severity } \\
\text { ( } 41 \text { children, } 23 \text { adults) }\end{array}$ & $21(22)$ & $12(18)$ & $\mathrm{F}_{1,62}=11.4, \mathrm{p}<.001^{*}$ \\
\hline $\begin{array}{c}\mathrm{ABC} \\
\text { ( } 41 \text { children, } 27 \text { adults) }\end{array}$ & $64(27)$ & $84(17)$ & $\mathrm{F}_{1,66}=31.5, \mathrm{p}<.001^{*}$ \\
\hline $\begin{array}{c}\text { DHI } \\
\text { ( } 42 \text { children, } 27 \text { adults) }\end{array}$ & $49(21)$ & $30(22)$ & $\mathrm{F}_{1,67}=45.5, \mathrm{p}<.001^{*}$ \\
\hline $\begin{array}{c}\text { DGI } \\
\text { ( } 30 \text { children, } 18 \text { adults })\end{array}$ & $20(3)$ & $23(1)$ & $\mathrm{F}_{1,46}=42.6, \mathrm{p}<.001^{*}$ \\
\hline $\begin{array}{c}\text { FGA } \\
\text { ( } 30 \text { children, } 18 \text { adults })\end{array}$ & $22(5)$ & $28(3)$ & $\mathrm{F}_{1,46}=62.9, \mathrm{p}<.001^{*}$ \\
\hline $\begin{array}{c}\text { Gait Speed } \\
\text { ( } 29 \text { children, } 17 \text { adults) }\end{array}$ & $1.02(.28)$ & $1.28(.23)$ & $\mathrm{F}_{1,44}=38.3, \mathrm{p}<.001^{*}$ \\
\hline $\begin{array}{c}\text { TUG } \\
\text { ( } 22 \text { children, } 16 \text { adults) }\end{array}$ & $9.7(2.5)$ & $7.8(1.8)$ & $\mathrm{F}_{1,36}=27.8, \mathrm{p}<.001^{*}$ \\
\hline $\begin{array}{c}\text { FTSTS } \\
\text { ( } 20 \text { children, } 16 \text { adults })\end{array}$ & $13.1(6)$ & $9.7(5)$ & $\mathrm{F}_{1,34}=15.9, \mathrm{p}<.001^{*}$ \\
\hline $\begin{array}{c}\text { SOT(Composite) } \\
\text { ( } 13 \text { children, } 9 \text { adults) }\end{array}$ & $48(19)$ & $71(13)$ & $\mathrm{F}_{1,20}=36.8, \mathrm{p}<.001^{*}$ \\
\hline $\begin{array}{c}\text { SOT Condition } 1 \\
\text { ( } 13 \text { children, } 8 \text { adults) }\end{array}$ & $83(13)$ & $92(4)$ & $\mathrm{F}_{1,19}=7.2, p=.015^{*}$ \\
\hline $\begin{array}{c}\text { SOT Condition } 2 \\
\text { ( } 13 \text { children, } 8 \text { adults) }\end{array}$ & $76(18)$ & $86(9)$ & $\mathrm{F}_{1,19}=5.3, \mathrm{p}=.033^{*}$ \\
\hline $\begin{array}{c}\text { SOT Condition } 3 \\
\text { ( } 13 \text { children, } 8 \text { adults) }\end{array}$ & $71(21)$ & $87(9)$ & $\mathrm{F}_{1,19}=7.8, \mathrm{p}=.012 *$ \\
\hline $\begin{array}{c}\text { SOT Condition } 4 \\
\text { ( } 13 \text { children, } 8 \text { adults) }\end{array}$ & $46(28)$ & $80(9)$ & $\mathrm{F}_{1,19}=27.2, \mathrm{p}<.001^{*}$ \\
\hline $\begin{array}{c}\text { SOT Condition } 5 \\
\text { ( } 13 \text { children, } 8 \text { adults) }\end{array}$ & $29(24)$ & $51(15)$ & $\mathrm{F}_{1,19}=21.6, \mathrm{p}<.001^{*}$ \\
\hline $\begin{array}{c}\text { SOT Condition } 6 \\
\text { ( } 13 \text { children, } 8 \text { adults) }\end{array}$ & $29(21)$ & $60(15)$ & $\mathrm{F}_{1,19}=32.0, \mathrm{p}<.001^{*}$ \\
\hline
\end{tabular}

ABC, Activities-specific Balance Confidence scale; DHI , Dizziness Handicap Inventory; DGI , Dynamic Gait Index; FGA, Functional Gait Assessment; TUG, Timed "Up\& Go"; FTSTS, Five Times Sit To Stand; SOT = Sensory Organization Test 
Table 4-3: Mean (SD) for the significant interaction effect between age group and time on dizziness severity and Sensory Organization Test (SOT) scores

\begin{tabular}{|l|c|c|c|c|}
\hline \multicolumn{1}{|c|}{ Outcome Measure } & \multicolumn{2}{|c|}{ Children } & \multicolumn{2}{c|}{ Adults } \\
\hline & Pre-treatment & Post-treatment & Pre-treatment & Post-treatment \\
\hline $\begin{array}{l}\text { Dizziness Severity } \\
(41 \text { children, 23 adults) }\end{array}$ & $26(22)$ & $7(11)$ & $21(20)$ & $20(25)$ \\
\hline $\begin{array}{l}\text { SOT Condition } 1 \\
(13 \text { children, 8 adults })\end{array}$ & $79(14)$ & $92(3)$ & $91(3)$ & $91(6)$ \\
\hline $\begin{array}{l}\text { SOT Condition } 2 \\
(13 \text { children, 8 adults })\end{array}$ & $72(21)$ & $89(5)$ & $83(11)$ & $83(13)$ \\
\hline
\end{tabular}

\subsection{DISCUSSION}

The primary finding of this study is that people who had persistent dizziness and balance dysfunction after having a concussion appear to have improved after vestibular rehabilitation.

Although many post-concussive symptoms, including dizziness and imbalance, may resolve within the first few weeks after the concussion, ${ }^{65,296}$ it is less likely that the participants in our sample fell in this category. Only $8 / 114(7 \%)$ of the participants in our sample had an initial evaluation within 3 weeks of the concussion, and the median number of 61 days (range 62566) between the most recent concussion and their referral to vestibular rehabilitation suggests that the symptoms did not resolve spontaneously, and consequently required intervention. Five participants in our sample had BPPV. Although BPPV has been shown to be common after concussion, it did not appear that way in our sample, perhaps because the extended length of time between the concussion and initial evaluation for vestibular rehabilitation. 
The intervention spanned a median of 4 vi sits and 33 da ys. The number of visits is comparable with other open-ended trials of vestibular rehabilitation for both peripheral and central causes of dizziness reported from the same clinic. ${ }^{300-301}$ However, the duration of care in this report was less than in other studies. It is not clear if the shorter duration of care represented truly faster recovery rates, or rather a change in frequency of treatment visits that enabled the therapists to progress the exercise program more quickly. However, this improvement in outcomes in the same number of visits over a shorter time period may indicate that patients referred to vestibular rehabilitation post-concussion may benefit from a higher frequency of visits at the beginning of care.

The patient recovery occurred across multiple domains, i.e. self- reports of dizziness severity, dizziness handicap (DHI), and balance confidence (ABC), as well as functional balance performance. The magnitude of improvement compares well with other types of vestibular disorders. ${ }^{269,300-302}$ Furthermore, the average magnitude of change was greater than the Minimal Clinically Important Difference (MCID) established for the DHI (18 pts), ${ }^{299}$ gait speed $^{303}(0.1$ $\mathrm{m} / \mathrm{s}$ ) and SOT composite score (10 pts). ${ }^{304}$ Although statistically-derived MCIDs have not been established for the other outcome measures, in our clinical experience, mean improvements of 20 for the ABC, 3 for the DGI, 6 for the FGA, suggest clinically significant changes. Without a control group, it is not possible to know the relative contributions of the vestibular rehabilitation program, concurrent medical management, and natural recovery toward the improved outcome measures. Furthermore, we were not able to determine how impaired the participants were immediately post-concussion, and therefore are unable to know how much improvement in outcomes may have already occurred prior to treatment. However, the finding is consistent with 
previous studies that have shown that vestibular rehabilitation may reduce dizziness and improve overall balance for individuals with concussion. ${ }^{34,44,234-235}$

The scores of dizziness severity and DHI at initial evaluation were similar to several other reports of persons with vestibular disorders, including central and peripheral dysfunction. ${ }^{300-301}$ However, our participant sample had qualitatively better scores in several of the functional gait and balance measures, including the DGI, TUG, and FTSTS, as well as the self reported $\mathrm{ABC}$. It is possible that the better gait and balance scores in the current study reflect the younger age distribution of this group. An age difference was found for several of the gait and balance measures, including FTSTS and FGA, so this explanation is plausible, but not definitive since normative data for these measures are lacking in children. In contrast, across all conditions, the SOT scores obtained from children during the initial evaluation (data not shown) were worse than scores obtained from adults with vestibular disorders, ${ }^{228,} 264,305$ and healthy children, ${ }^{297,} 306$ providing additional evidence of dysfunctional sensory integration with concussion in children. 295,307

In order to assess if age affected the amount of improvement, the interaction between time and age was examined. Only 3 of the measures demonstrated significant interactions: dizziness rating and SOT Conditions 1 a nd 2. The significant interaction for the dizziness severity revealed that children had a greater improvement in symptom severity despite having slightly worse ratings at the initial evaluation. Closer inspection of the dizziness severity data for the adult group showed that despite the improvement of dizziness in many participants, the large variability in the scores, especially post-treatment scores may attribute to the overall lack of time effect on dizziness for the adults. The significant interaction effect in SOT Conditions 1 and 2 indicates greater improvement in scores in children compared with adults. The adults had a 
narrow range of scores that were within normal limits for SOT Condition 1 whereas the distribution of the scores for SOT Condition 1 were more dispersed in children, which allowed for greater recovery. In SOT Condition 2, children again had greater room for improvement because of lower initial scores, but it is also surprising that the adults' scores did not increase to normal values. Overall, the results of posturography analyses should be interpreted cautiously, as they were limited by the low number of participants (13 children / 8 adults). However, the lack of interaction between time and age in other outcome measures of DGI, FGA, gait speed, FTSTS, and SOT suggests that these gait and balance measures could be used to track the recovery after vestibular rehabilitation for both adult and children populations. It would be of a great interest if future studies investigate the responsiveness of gait and balance measures in patients with mTBI.

Another aim of the study was to examine if age impacted the overall level of symptoms and the amount of recovery. There were age-related differences in DHI, FGA, and FTSTS scores. The significantly lower scores on the DHI in children compared with adults may support the notion that the perception of dizziness handicap is different between children and adults. ${ }^{243}$, ${ }^{293}$ However, at the time of initial evaluation, the dizziness severity was not different between the 2 groups. This apparent contradiction may be explained by items in the DHI that are not applicable to children (e.g. Does your problem interfere with your job or household responsibilities) and by lack of items that relate dizziness to the functional difficulties that the children are experiencing. ${ }^{293}$ Although the DHI has not been validated for use with children, we used it because there were no other alternatives that assess the impact of dizziness on the functional activities of children, and we felt that most of the items would respond to changes that occurred during vestibular rehabilitation. The lower FTSTS scores in children (by 4 s) are 
probably not related to any concussion-related factors, but rather explained by greater physical fitness and agility. In addition, significantly higher FGA scores in children (by 2 points) may reflect abilities that are not related to the concussion severity. Furthermore, a difference of two points between groups may not be clinically meaningful.

\subsection{LIMITATIONS AND FUTURE DIRECTIONS}

Although we believe that this study added to our understanding of the effects of vestibular rehabilitation after concussion, the results of this study were limited by the retrospective nature of the data and the lack of control group. Owing to the retrospective nature of the study, many limitations were identified; first, we were not able to reliably report the immediate markers of concussion severity such as loss of consciousness (LOC), amnesia and confusion, and we were not able to investigate if the presence of these markers could predict poorer outcomes. Second, although acknowledged by athletic trainers as an essential tool in concussion assessment, ${ }^{125,308-}$ 311 symptom checklist was not implemented as a $p$ art of vestibular assessment. Therefore, we were not able to examine the symptoms severity and symptoms recovery in our sample. Third, since there were no complete vestibular battery test, we were not able to place our participants in one of the dizziness groups previously described by Hoffer et al. ${ }^{34}$ Fourth, the study was also limited by some missing data and a low number of participants who completed pre- and posttesting, especially the SOT.

We strongly recommend the use of the symptom checklist as a part of vestibular evaluation as it allows the therapist do document the symptomatic severity of the participants and allow the therapist to track the symptoms recovery by examining the difference in symptom 
severity between the initial sideline symptom checklist, which is commonly used in the field of athletic training and subsequent checklists.

We also recommend a multidisciplinary approach in which neuropsychologist, physical therapist, athletic trainer can bring their expertise and work together to comprehensively mange the concussion. Knowing that there are modifiers for concussion management, ${ }^{70}$ we recommend having a profile for the concussion patient in which all the tests for the different domains (Symptoms, neurocognitive and balance) at all evaluation points (baseline, immediately post concussion, and throughout recovery) are documented in a systematic manner and kept accessible to any member of the team.

Future directions for research should include replication of this study in a well controlled prospective design with a control group.

\subsection{CONCLUSION}

Vestibular rehabilitation therapy may reduce dizziness severity and improve gait and balance performance after concussion in both children and adults who have persistent symptoms that do not resolve with rest. Future directions may include replication of this study in a prospective and controlled design. 


\subsection{THIRD AIM}

\subsection{INTRODUCTION}

Reports of dizziness and imbalance are prevalent in individuals who have had a concussion.

Twenty-three to eighty-one percent of persons post concussion report dizziness in the first days post concussion. Estimates of the prevalence of persistent dizziness after mTBI varies widely from $16-18 \%$ at three months, ${ }^{170,181} 1.2 \%$ at 6 months ${ }^{182}$ to $32.5 \%$ at five years. ${ }^{183}$

Persistent balance problems have also been reported three weeks after concussion. ${ }^{18,171}$

Vestibular rehabilitation is a key component to the management of dizziness and balance disorders resulting from vestibular system dysfunction, either peripherally or centrally. ${ }^{226-231}$

Despite the high incidence of dizziness and balance dysfunction in people who have had a concussion, reports of vestibular and balance rehabilitation in management of concussion are sparse. ${ }^{33-34,39,44}$ Previous studies have shown that vestibular rehabilitation reduces dizziness and improves overall balance for individuals with head injury. ${ }^{33-34,39,44,249}$

The accepted standard of care for vestibular rehabilitation is to use a problem-oriented approach in which impairments and functional limitations are identified during the initial evaluation, and customized exercises are prescribed to address the individual's specific problems, while accounting for the pathology and other co-morbidities. ${ }^{244}$ General guidelines for exercise prescription and progression are available in Herdman et al, ${ }^{244}$ and more specific 
programs have been documented for prospective clinical trials. ${ }^{244}$ Outside of these descriptions of authoritative practice guidelines, there have been no reports of how vestibular rehabilitation therapists actually use these principles in practice. An understanding of what exercises have been prescribed for individuals with dizziness and imbalance after concussion may be useful for several reasons. Assuming that the physical therapists adhered to the problem-oriented approach described above, the study can provide a d etailed picture of the specific impairments and functional limitations encountered by the individuals with concussion and the path by which they returned to their pre-morbid status. Moreover, by understanding the prescription and progression patterns provided by expert clinicians, physical therapists new to prescribing vestibular rehabilitation exercises for patients with concussion can use this information to better understand how to start and then make the exercises more challenging. Additionally, therapists can use the framework of exercise categories, exercise groups and exercise modifiers to better document the detailed prescription pattern of vestibular rehabilitation exercises. Consequently, the purpose of this study is to describe exercise prescription patterns in patients treated with vestibular rehabilitation exercises.

\subsection{METHODS}

\subsubsection{Participants}

A retrospective chart review of the 114 participants referred to a tertiary vestibular rehabilitation clinic for vestibular rehabilitation after being diagnosed with concussion. Five patients did not have an indication for vestibular rehabilitation therapy, four patients were diagnosed with Benign 
Positional Paroxysmal Vertigo (BPPV) and were successfully treated using the modified Epley Canalith Repositioning Maneuver ${ }^{312}$ without need for home exercises. One patient did not return for subsequent visit. A total of 104 participants $(66 \mathrm{~F} / 38 \mathrm{M}$, mean age $24 \mathrm{y}$, SD $19 \mathrm{y})$ received a computer-generated home exercise program (HEP) of vestibular rehabilitation exercises after being diagnosed with concussion. Results of the intervention were previously reported. ${ }^{249}$

Participants were referred for a median of $58(6-1,149)$ days after the concussion episode. The duration of the vestibular rehabilitation intervention was a median of 33 (range 7181) days, encompassing a median of 4 (range 2-13) visits. The HEP was designed by eight physical therapists with at least three years experience in vestibular physical therapy.

\subsubsection{Procedure}

Each of the computer-generated exercise handouts (Visual Health Information (VHI), WA, U.S.A) that was placed in the chart was reviewed by one of the authors (PS) and each exercise was classified according to general exercise categories that address common areas of dysfunction in individuals with vestibular and balance disorders. VHI software has a pre-determined set of exercises that allows therapists to make modifications for the initial exercise prescription. The software also allows therapists to create new exercises and to make changes to progress the different exercise prescriptions.

There are five main exercise categories: 1) Eye-Head Coordination 2) Sitting balance 3) Standing Static balance (i.e. feet-in-place), 4) Standing Dynamic balance (feet moving, but not walking), and 5) Ambulation. Each one of these categories consisted of different exercise types.

A brief description of the exercise categories and types is included below. 
1) Eye-Head Coordination exercises: This exercise category contains many exercise types that involve movement of head and/or eyes for the purpose of vestibulo-ocular reflex (VOR) gain adaptation, symptom habituation, or oculomotor re-education. The exercises include: VORx1, VORx2, VOR cancellation, convergence, smooth pursuits, anticipatory gaze shifts, imagined target, and saccades. ${ }^{250}$

2) Sitting balance exercises: The patient maintains balance while sitting upright, weight shifting from side to side, or bouncing.

3) Standing Static balance exercises: The patient stands with feet in place while upright or weight shifting. The patient can be asked to stand on one leg, stand on a rocker board or stand with one foot on a step. This category also includes the sit-to-stand exercise.

4) Standing Dynamic balance exercises: The patient stands and moves without walking.

The patient might march in place, step forward or backward, step to the side, step up or down, or turn around.

5) Ambulation exercises: The patient walks forward, backward, on stairs, with turns and practice braiding (i.e. side stepping in an over and under pattern), skipping, jogging and running.

For each type of exercise, a universal set of 10 modifiers was used to describe other characteristics of the exercise (Table 5-1): (1) the posture in which the exercise is performed, (2) the type of support surface, (3) the size of the base of support, (4) the positioning of the trunk and (5) arms, (6) the direction of head movements, (7) the direction of whole body movements, (8) the visual input, (9) the presence or absence of the dual cognitive task and (10) any other special circumstances, such as target distance (near or far) when performing VORx1 exercise.

The frequency and duration of time prescribed per exercise were also recorded. Frequency is recorded in terms of the number of times it is performed per day, and the duration 
of the exercise is given by length of time or number of repetitions. Finally, the intensity of the exercise can be described in terms of speed of movement or change in symptom rating.

Note that the intensity may not have relevance for some categories, for instance Sitting or Static Standing balance.

Table 5-1: The exercise modifiers used the vestibular rehabilitation exercises

\begin{tabular}{|c|c|}
\hline Modifier & Choices \\
\hline (P) Posture & 1: Sitting, 2: Standing, 3: Walking, Not Applicable/Not Specified (NA/NS) \\
\hline (S) Surface & 1: Level, 2: Foam, 3: Uneven, 4: Obstacle, 5: Stairs, 6: Ramps, NA/NS \\
\hline (B) Base of support & 1: Feet-apart, 2: Feet-together, 3: Semi-tandem, 4: Tandem, NA/NS \\
\hline (T) Trunk position & 1: Upright, 2: Leaning, 3: Rotated, NA/NS \\
\hline (A)Arm position & $\begin{array}{l}\text { 1: Weight bearing, 2: Close to body, 3: Away from body, 4: Reaching, } \\
\text { Carrying, 6: Picking up objects, 7: Juggling, NA/NS }\end{array}$ \\
\hline $\begin{array}{l}\text { (H)Head movement } \\
\text { direction }\end{array}$ & 1: Still, 2: Yaw, 3: Pitch, 4: Roll, NA/NS \\
\hline (V)Visual input & 1: Eyes closed, 2: Eyes open, 3: Complex patterns, NA/NS \\
\hline $\begin{array}{l}\text { (D) Direction of whole } \\
\text { body movements }\end{array}$ & 1: Anterior-posterior, 2: Medial-lateral, 3: Multi-directional, NA/NS \\
\hline (C) Cognitive dual task & 1: Yes, 2: No \\
\hline Special circumstances & e.g. note if the VORx1 exercise was performed with a near or far target \\
\hline
\end{tabular}




\subsubsection{Analysis}

In order to provide an understanding about the patient's initial presentation and the progression for a sp ecific exercise type, the visit during which each new exercise was prescribed was recorded. An exercise qualified as a new exercise if at least one modifier was different than the previous prescriptions. Progression was defined as performance of the same exercise type, but under more challenging conditions (e.g. progressing VORx1 from standing with feet apart to feet together or walking). Frequency counts of the most common exercise categories and most common exercise types within each category (constituting 95\% of each category) were recorded.

Exercises provided to the participants as a home exercise program were analyzed, rather than the exercises performed during the clinic visit, because the documentation was more uniform and presumably reflected the most important activities that each patient needed to work on. Throughout the results, and unless specified otherwise, the percentages were relative to the overall number of participants who received a HEP (i.e. $n=104)$.

\subsection{RESULTS}

A total of 104 participants had a home exercise program (HEP) prescription. Out of the sum total of 411 patient visits, a printed HEP sheet was located for 335 visits (82\%). It is not known in the remaining $18 \%$ of the visits, if the participants were given a new HEP without being placed in the chart, or if the therapist wanted the patient to continue with the same HEP. The 76 missing home exercise notes were distributed among 40 participants. There were no differences in age, gender and time between concussion and vestibular rehabilitation between this group with 
missing data and the group without missing data; however, the group with missing data had a longer treatment duration $(48 \pm 29$ days v. $26 \pm 16$ days, $\mathrm{p}<0.001)$.

Table 5-2 summarizes the frequencies for the most common exercise categories and exercise types, prescribed throughout the duration of the intervention as well as for the first visit only. Eye-Head Coordination exercises were the most commonly prescribed category, including $95 \%$ of the participants who had received a HEP. The VORx1, VOR cancellation, convergence and VORx2 were the four most common exercises in this category, respectively. The Standing Static balance category was the second most common category of exercises, prescribed in $88 \%$ of participants; the most frequent were standing upright on level and foam surfaces, single leg stance, weight-shifting exercises in various directions, and sit-to-stand. The Ambulation category was the third most commonly prescribed category ( $76 \%$ of participants), with forward ambulation followed by backward ambulation, and walking with turns. To accompany these commonly prescribed exercises, we have detailed the most common frequency and duration as specified by the physical therapists. 
Table 5-2: Summary of the most common prescribed exercises throughout therapy and during the first visit in number of subjects (Percentage of subjects)

\begin{tabular}{|c|c|c|c|c|}
\hline Exercises & $\begin{array}{l}\text { Throughout } \\
\text { Therapy }\end{array}$ & First Visit & $\begin{array}{r}\text { Frequency } \\
\text { (times/day) }\end{array}$ & Duration \\
\hline Eye- Head Coordination & $99(95 \%)$ & $89(86 \%)$ & & \\
\hline VORx1 & $92(88 \%)$ & $75(72 \%)$ & 3 & $60 \mathrm{~s}$ \\
\hline VOR cancellation & $67(64 \%)$ & $31(30 \%)$ & 2 & 10 reps \\
\hline Convergence & $30(29 \%)$ & $21(20 \%)$ & 2 & 10 reps \\
\hline VORx2 & $9(9 \%)$ & $1(1 \%)$ & 1 & $30 \mathrm{~s}$ \\
\hline Standing Static & $92(88 \%)$ & $69(66 \%)$ & & \\
\hline Standing Upright & $87(84 \%)$ & $67(64 \%)$ & 2 & $30 \mathrm{~s}$ \\
\hline Single leg stance & $29(28 \%)$ & $7(7 \%)$ & 4 & $30 \mathrm{~s}$ \\
\hline Weight Shift & $15(14 \%)$ & $2(2 \%)$ & 1 & 10 reps \\
\hline Sit To Stand & $14(13 \%)$ & $11(11 \%)$ & 1 & 10 reps \\
\hline Ambulation & $79(76 \%)$ & $43(41 \%)$ & & \\
\hline Forward Ambulation & $76(73 \%)$ & $42(40 \%)$ & & \\
\hline Feet Apart, yaw head turns & $64(62 \%)$ & $31(30 \%)$ & 1 & 20 head turns \\
\hline Feet Apart, pitch head turns & $29(27 \%)$ & $12(12 \%)$ & 1 & 20 head turns \\
\hline Tandem, head still & $33(32 \%)$ & $9(9 \%)$ & 1 & 20 feet \\
\hline Backward Ambulation & $44(42 \%)$ & $10(9 \%)$ & & \\
\hline Feet Apart, head still & $18(17 \%)$ & $8(8 \%)$ & 1 & 20 feet \\
\hline Feet Apart, yaw head turns & $14(13 \%)$ & $0(0 \%)$ & 1 & 20 head turns \\
\hline Tandem, head still & $21(20 \%)$ & $3(3 \%)$ & 1 & 20 feet \\
\hline Ambulation with Turns & $19(18 \%)$ & $2(2 \%)$ & 1 & Every 5 steps \\
\hline
\end{tabular}




\subsubsection{Preferred Prescription Patterns}

Even though the number of potential exercises is large due to the combination of modifiers that could be used, the physical therapists demonstrated preferred prescription patterns that contained a small subset of the potential combinations. For example, examination of these patterns revealed that the VORx1 exercise was usually prescribed in both the yaw and pitch planes. For each patient, it was further customized by changing the posture, size of base of support and visual input. Therefore, the therapists usually did not alter the following modifiers during the VORx1 exercise: surface, trunk position, arm position, direction of whole body $\mathrm{m}$ ovement or involvement of cognitive dual task. The specific patterns for the VORx1 exercise will be presented in more detail in the section on progression.

The VOR cancellation exercise was customized primarily by changing the posture and/or the base of support (BOS). As with the VORx1, VOR cancellation was prescribed in both yaw and pitch planes. In $38 \%$ of the participants who received a HEP, VOR cancellation was prescribed in the standing position on a level surface (most with feet apart). While in the standing position, the target was either held in one hand while the arm was moving (e.g. a playing card) or tossed between hands (e.g. a ball). This exercise was also performed while walking ( $37 \%$ of participants), also with targets that were tossed or held in the hand. VOR cancellation was prescribed in the sitting position for $18 \%$ of participants, always while having the target held in hand.

For the Standing Static balance exercise category, the standing upright exercise was customized and/or progressed by changing the surface, BOS, direction of head movement and the visual input modifiers. The most common surface was a level one, which was prescribed in $74 \%$ of the participants receiving a HEP. Participants were instructed to stand with feet together 
(46\%), followed by standing tandem and semi-tandem (34\% and 31\% respectively). Across these three BOS's, the exercise was most commonly prescribed with head still and eyes closed. When the exercise was given with head movement in the yaw direction $(n=45)$, the eyes were open in most cases $(n=41)$. When performed on a foam surface $(37 \%)$, the standing upright exercise was most commonly prescribed with feet apart followed by feet together ( $27 \%$ and $8 \%$ respectively).

When performed with feet apart, it was most commonly performed with head still and eyes closed.

The most common modifiers of the Ambulation exercises were the BOS and direction of head movement. Forward ambulation was the most common prescribed exercise in $73 \%$ of the participants. The majority of the participants performed the exercise with feet apart (68\%).

Tandem ambulation was the second most common forward ambulation (34\%). Across the different BOS's the exercise was prescribed with yaw head movement most frequently (62\%), then with pitch head movement and no head movement ( $28 \%$ and $12 \%$ respectively).

\subsubsection{Progression}

The VORx1 exercise can be used as an example of how exercises were progressed (Table 5-3).

The initial exercise prescription was given most commonly in standing with feet apart (40 participants), followed by s tanding with feet together (29 participants) and sitting (21 participants). For the participants who were prescribed VORx1 in sitting, 11 participants were progressed to standing with feet apart, 6 participants to standing with feet together, 2 to walking and 4 participants did not progress any further. During the final prescription of VORx1, 31, 30 and 19 participants were instructed to perform the exercise during walking, standing with feet 
together and standing with feet apart, respectively. Fewer numbers of participants were given their last VORx1 progression in the semi-tandem and tandem position (6 and 2 participants respectively).

Table 5-3: Progression patterns for the VORx1 exercise $†$

\begin{tabular}{|c|c|c|c|c|c|}
\hline \multicolumn{6}{|c|}{ Posture } \\
\hline & Sitting & $\begin{array}{c}\text { Standing } \\
\text { Feet Apart }\end{array}$ & $\begin{array}{c}\text { Standing Feet } \\
\text { Together }\end{array}$ & $\begin{array}{c}\text { Standing } \\
\text { Tandem/ST }\end{array}$ & Walking \\
\hline Initial prescription & 21 & 40 & 29 & 1 & 1 \\
\hline \multicolumn{6}{|l|}{ Progression(s) } \\
\hline Sitting & & - & 1 & - & - \\
\hline Standing Feet Apart & 11 & & - & - & - \\
\hline Standing Feet Together & 6 & 19 & & - & - \\
\hline Standing Tandem/ST & - & 2 & 7 & & 2 \\
\hline Walking & 2 & 10 & 17 & 3 & . \\
\hline Standing Other & - & 2 & 2 & - & - \\
\hline Walking Other & - & - & - & - & 2 \\
\hline Final prescription & 4 & 19 & 30 & 8 & 31 \\
\hline
\end{tabular}

\subsection{DISCUSSION}

Based on the exercises that were prescribed, we can infer that most of the impairments in these individuals with concussion were in three domains; Eye-Head Coordination, Standing Static balance, and Ambulation. The exercises that were given to the participants in this study are consistent with those prescribed in other research studies involving vestibular rehabilitation. ${ }^{228-}$ 229, 313-314 Moreover, although the previous studies of vestibular rehabilitation in patients with 
post concussion symptoms did not provide a detailed description about their vestibular rehabilitation program, the use of VORx1 and "positional exercises" has been reported. ${ }^{34}$

Impairments in the Eye-Head Coordination have been reported after brain injury, and may result from disruption in the vestibulo-ocular reflex (VOR) ${ }^{45}$ or reflect increased symptoms with head and eye movement. ${ }^{315}$ Vestibular adaptation exercises that improve VOR gain will consequently improve gaze stabilization during head movement. ${ }^{244,313}$ Eye-Head Coordination exercises can also be prescribed for habituation purposes if the patient is found to have symptom provocation independently of any reduction in VOR gain. In the current sample of participants, $95 \%$ were found to have impairments in Eye-Head Coordination. Because most of the participants did not have formal vestibular function testing, it is not certain how many participants had reduced VOR gain. However, most of the participants had symptom provocation with eye and head movements.

Several studies have reported different balance and ambulation impairments in patients with post-concussion symptoms. Despite the evidence that impairments in the static balance spontaneously resolve within the first 3-5 days after concussion, ${ }^{30-31} 88 \%$ of the current participants were found to have impairments in Standing Static exercises at least 6 days after the concussion. In addition, patients may exhibit slower gait velocity, ${ }^{15,28,152}$ shorter stride length, ${ }^{150}$ and wider step width. ${ }^{29}$ In the current sample of participants, Ambulation impairments were found in $76 \%$ of the participants.

Ninety percent of participants who had difficulties in the domain of Eye-Head Coordination received a HEP to address gaze stabilization during their first visit (Table 5- 2). Of the participants who had standing static balance difficulties, $75 \%$ received a HEP in the Standing Static balance category during the first visit. However, only $54 \%$ of the participants who had 
difficulties during walking were given ambulation exercises during their first visit. These results suggest that the Eye-Head Coordination category is the domain that is usually addressed first by the expert clinicians during vestibular rehabilitation for patients post concussion. Several factors may account for why Ambulation exercises were not emphasized during the first visit. First, it is common for persons with vestibular disorders to become symptomatic before the Ambulation evaluation is complete. ${ }^{250}$ Second, the therapist may have identified gait impairments but decided to address the Eye-Head Coordination and Standing Static balance problems first to instill confidence and make sure the participant performed the exercises safely before addressing the more dynamic balance deficits (i.e. during ambulation). Others have suggested using a less aggressive pattern of progression for patients with post concussion symptoms during vestibular rehabilitation. ${ }^{45}$ Although the reasons for prescription patterns have not been verified through the current data, these above mentioned issues are frequently seen in the management of patients with vestibular disorders and warrant a frequent re-evaluation for patient's status in order to determine the current impairments and functional limitations throughout the course of vestibular rehabilitation. ${ }^{250}$

The analysis revealed important observations about the exercise prescription and progression patterns. For Eye-Head Coordination exercises, the most important modifiers were the posture and base of support. A typical pattern of progression for the VORx1 exercise would be from sitting to standing with feet apart, to standing with feet together, to walking. By varying the combinations of posture and the size of base of support, the patient must learn to adjust for natural body movements that occur while coordinating the eye and head movements during typical daily activities. VOR cancellation is needed to follow moving objects while the head is synchronously moving in the same direction. Posture was the most important modifier to be 
changed during VOR cancellation prescription. The exercise was mainly performed in postures that match the scenarios in which the suppression of VOR is needed in real life (i.e. standing and walking). For participants who were more symptomatic, the exercise was prescribed while sitting and progressed to standing and walking after the symptoms improved (data not shown).

Participants received the VORx2 exercise as part of the progression less frequently (9\%) than is typically prescribed for people with unilateral vestibular hypo-function. Several reasons may exist to explain this. The participants may not have returned for enough visits to begin this exercise, because their symptoms improved and they were discharged. In other cases, the participants may still have been highly symptomatic with the VORx1 exercise.

Because of the retrospective study design, we were not able to record the explicit rationale for the therapists' prescription. All of the therapists who prescribed the exercises were trained in the customized problem-oriented approach that is considered to be the standard intervention for vestibular rehabilitation. ${ }^{244}$ Therefore, we can assume that the general rationale for the exercise prescription in each case $\mathrm{w}$ as developed using the same framework of 1) identifying the impairments during the evaluation, 2) prescribing a specific initial exercise to address that impairment safely, and 3) progressing the exercise by increasing difficulty so that the activity can be done in a functional manner. Although the current design cannot specifically address "why" the exercises were given, we believe knowing "what" was prescribed by expert therapists is an important step in understanding the management of these individuals. Given the extreme shortcomings in the published literature about the vestibular rehabilitation exercises for individuals post concussion, we believe that this manuscript is useful for clinicians who are starting vestibular rehabilitation exercises because the exercise prescription and progression patterns included in this manuscript is more detailed from previous studies that have commented 
on the vestibular rehabilitation exercises post concussion. ${ }^{33-34,44}$ Consequently, if a therapist has a basic understanding of the general principles of vestibular rehabilitation, they can now see how other therapists initially began the program and then progressed the participants. For example, the results of this study suggest that during the initial visit, a therapist may want to concentrate on assessing Eye-Head Coordination exercises and Standing Static balance activities, and when indicated, prescribe exercises in these domains. Initially, the VORx 1 exercise was prescribed most frequently in standing with feet apart. In later visits, the participants progressed to perform additional Eye-Head Coordination exercises, in more destabilizing postures, and also perform more Ambulation exercises. Future studies should incorporate a prospective design and determine both elements of prescription pattern (i.e. the "what" and "why").

Additionally, we were not able to report the intensity of the prescribed exercises; this is attributed in part to the retrospective nature of our study in which the intensity was not specified in the software that was used to generate the home exercise program sheets. In the case of EyeHead Coordination exercises, participants were generally instructed to move their head at a speed that caused their symptoms to increase slightly. Nonetheless, we believe intensity should be more formally quantified in the prescription of vestibular rehabilitation exercises, in cases where it applies. For instance, in the case of Eye-Head Coordination exercises, the speed of head movements (or eye movements) is not well described, and is difficult to monitor. Perhaps wearable sensors could be developed to assist in this area in which the speed of head movement is recorded. Another potential way to prescribe the intensity, especially in the case of symptom provocation, is by having the participant perform the exercise until they reach a certain level of symptom severity on a visual or verbal analog scale. For standing dynamic exercises, the intensity could be prescribed as the number of repetitions per minute, and increased to a higher 
intensity during the progression. For Ambulation exercises, intensity could be prescribed as the gait speed, or speed of head movements performed during gait.

While the progression of aerobic and resistance exercises is typically based on increasing the intensity or volume of the same exercise type, vestibular rehabilitation exercises are often based on subtle variations of the exercise types that are not able to be classified using the F.I.T.T (frequency, intensity, time and type) principle of the American College of Sports Medicine (ACSM). The current system was designed to facilitate the reporting of exercises commonly used during vestibular rehabilitation. ${ }^{244,316}$ The 5 main exercise categories and exercise types within each category were loosely based on general exercise categories that address common areas of dysfunction in individuals with vestibular and balance disorders. It is important to note that the patients with dizziness and balance disorders also may have been given range of motion and strengthening exercises as a part of their home exercise program. However, the prescription frequency of these exercise types was much lower than the vestibular rehabilitation exercises.

One of the limitations encountered in the study was having missing home exercise programs. The group with missing data did not differ from the group with complete data in age, gender, and time since concussion; therefore, systematic bias is less likely to have had occurred in prescriptions that may have been based according to age and gender. The group difference in treatment duration may indicate that there was greater opportunity to have data missing, or support the notion that the therapist may have asked the more impaired participants (i.e. longer treatment duration) to continue the same HEP without documenting that in the chart. 


\subsection{CONCLUSION}

Individuals with dizziness and imbalance post concussion may exhibit impairments in Eye-Head Coordination, Standing Static balance and Ambulation. The exercises prescribed by expert clinicians are comparable to the exercises prescribed for individuals with unilateral vestibular hypo-function. Even though the number of potential exercises is nearly limitless due to the number of modifiers, the physical therapists demonstrated preferred prescription patterns that limited the number of modifiers used. By knowing the preferred prescription and progression pattern of exercises employed by e xpert physical therapists, other clinicians initiating a vestibular rehabilitation treatment program for individuals post-concussion may have a foundation to guide their intervention. 


\subsection{FOURTH AIM}

\subsection{INTRODUCTION}

Mild traumatic brain injury, or concussion, can result in a variety of symptoms and physical and cognitive impairments. Post- traumatic headache is the most common symptom after concussion. ${ }^{171,174-175}$ Prevalence of initial headache after concussion has been reported to range between $43 \%$ to $86 \%$ of patients. ${ }^{18,171,176}$ Persistent headache is also reported in a period up to three months after concussion. ${ }^{170,172}$

Dizziness is also a frequent symptom after concussion, with $23 \%$ to $81 \%$ of persons reporting dizziness in the first days following concussion. ${ }^{18,}{ }^{181}$ Of the $61 \%$ who reported dizziness in the initial days after concussion in one study, $41 \%$ reported mild dizziness, $16 \%$ reported moderate dizziness, and $4 \%$ reported severe dizziness. ${ }^{18}$ Dizziness at the time of concussion was associated with a 6.34 odds ratio $\left(95 \% \mathrm{CI}=1.34-29.91, \chi^{2}=5.44, \mathrm{P}=.02\right)$ of a protracted recovery (i.e $>21$ days) from concussion in a sample of high school football players. ${ }^{191}$

Estimates of the prevalence of persistent dizziness after mTBI varies widely from 16$18 \%$ at three months, ${ }^{170,181} 1.2 \%$ at 6 months ${ }^{182}$ to $32.5 \%$ at five years. ${ }^{183}$ Self- reported balance problems have been reported by as many as $60 \%$ of persons post- concussion. ${ }^{171}$ Although some studies have suggested that self- reported balance problems usually resolve in the first days after 
concussion, ${ }^{31,} 144,146$ patients may exhibit poor balance despite not reporting balance problems. $^{184}$

In examining the recovery process after concussion, clinicians and researchers assess a

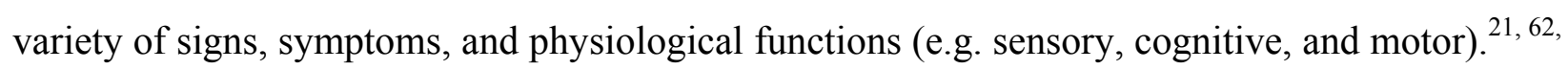
80, 124, 141 The diversity in measurements used to assess individuals with concussion ha ve enhanced our understanding of the multi-faceted nature of the effects after concussion, and improved the sensitivity of the assessment battery available to record functioning in individuals with concussion. ${ }^{20} \mathrm{~A}$ few studies have concluded that cognitive and motor effects of concussion resolve differently after concussion. ${ }^{16,216}$ Parker et al. have found a differential rate of recovery by comparing the recovery pattern in ImPACT composite scores and different dynamic motor tasks. $^{216}$

The lack of correlation between recovery of measures of different domains has been a subject of debate. ${ }^{14,20-21,48,104}$ For instance, while some view the lack of perfect correlation between performance measures and self- report symptoms as an indication for the lack of sensitivity in performance measures ${ }^{48}$ or an indication for the inaccuracy of self- report symptoms, ${ }^{21}$ others speculate that they may represent fundamentally different neurobehavioral processes, or exhibit different recovery trajectories, and therefore they should not be expected to correlate in all cases. ${ }^{14,20,104}$ Lovell et al. has concluded that post-concussion symptoms are a result of combinations of neurocognitive deficits and other factors (e.g. vestibular dysfunction), and therefore, the correlation (or lack thereof) between symptoms and neurocognitive testing is expected to be less than perfect. ${ }^{104}$

Despite the conceptual debate about the reasons behind the correlation (or lack thereof) between measurements from different domains, examining the relationship between 
measurements may enhance our understanding about the multi-faceted nature of concussion effects and recovery. Collins et al. revealed a correlation between the neurocognitive testing scores and the severity of headache post concussion. ${ }^{140}$ Preliminary evidence from a study that examined self-report symptoms revealed that "balance problems" were significantly correlated with "feeling mentally foggy", "difficulty remembering", and "difficulty concentrating". ${ }^{21}$ In addition, these symptoms were also associated with decreased cognitive performance (i.e. ImPACT composite scores). In the same study Broglio et al. examined the relationship between self- report "dizziness" and "balance problems" and Sensory Organization Test (SOT). The study found a significant correlation between change in the total score of SOT to balance problems. ${ }^{21}$

The purpose of this study is to examine the relationship between self-reported symptoms, neurocognitive performance and balance performance in individuals referred to vestibular physical therapy after concussion, and to examine the relationship between recovery in gait and balance measures and neuropsychological recovery made over the course of vestibular physical therapy.

\subsection{METHODS}

\subsubsection{Participants}

A retrospective case series was performed of 114 consecutive participants who were referred between 2006 and 2008 to a tertiary balance center for vestibular physical therapy after being diagnosed with a concussion. The study was approved by the institutional review board (IRB). 


\subsubsection{Outcome measures}

Self-report and performance gait/ balance measures were administered during the first physical therapy visit as well as at weekly and monthly intervals. The time points considered for this report include the initial evaluation and discharge scores. If a measure was not recorded at the time of initial evaluation or discharge, the assessment at the time point closest to the initial evaluation or discharge was used.

\subsubsection{Self report balance measures}

Participants were asked to rate their current dizziness severity on a verbal scale from 0-100, where 0 means no dizziness and 100 means maximum dizziness. Verbal anchors relating to severity of dizziness (e.g. slight, mild, moderate, severe) were provided for the scale.

The Activities-specific Balance Confidence scale (ABC) is a questionnaire used to assess the respondent's level of confidence that they would not lose their balance while performing 16 functional activities. The highest possible score of 100 suggests maximum confidence and a score of 0 suggests no confidence. ${ }^{255}$

The Dizziness Handicap Inventory (DHI) is an instrument used to assess the individual's handicap due to their dizziness in 25 items relating to physical, emotional, and functional domains. The highest overall score on the test is 100 and higher scores indicate greater handicap resulting from dizziness. ${ }^{299}$ 


\subsubsection{Balance performance measures}

The Dynamic Gait Index (DGI) is an eight item instrument that assesses the ability to walk with head turns, changes of speed, and around obstacles. The scale for each item ranges from 0-3, where 0 means severe impairment and 3 means normal. The highest possible score is $24 .^{256}$

The Functional Gait Assessment (FGA) is a 10-item test based on the DGI. The maximum score is 30 . Higher scores indicate better performance. ${ }^{263}$

While participants were asked to walk at their comfortable speed, gait speed was timed over 4 meters course using stopwatch.

The Timed "Up\& Go" (TUG) is a timed test during which the participant stands from a chair, walks three meters at their normal walking speed, and returns to the chair. ${ }^{258}$

The Five Times Sit To Stand (FTSTS) requires participants to stand-up and sit down from a standard height chair five times as quickly as possible. The participants were asked to complete the task with their hands crossed on their chest. ${ }^{259}$

Dynamic Computerized Posturography: Participants performed the Sensory Organization Test (SOT, Neurocom, Inc.) under six different sensory conditions:1) eyes open, fixed support; 2)eyes closed, fixed support; 3)sway-referenced vision, fixed support; 4) eyes open, swayreferenced support; 5) eyes closed, sway-referenced support; 6) sway-referenced vision and support surface. Three $20 \mathrm{~s}$ trials were performed for each condition. The highest theoretical equilibrium score is 100 which indicates no sway; losses of balance were graded as zero.

Average scores for each condition were recorded, and the composite score was calculated using a weighted average of the individual trials. 


\subsubsection{Neurocognitive and symptom measures}

During the time participants were seen for vestibular physical therapy, participants received neuropsychological assessments that included repetitive administration of the Immediate Postconcussion Assessment and Cognitive Testing (ImPACT). ${ }^{24}$ ImPACT is a co mputer administered software program that consists of a neurocognitive testing battery and postconcussion symptom (PCS) scale. ${ }^{24}$ The neurocognitive testing of ImPACT measures different areas of cognitive functioning including attention, processing speed, reaction time, and memory.

The ImPACT neurocognitive battery uses six test modules (Word Memory, Design Memory, X's and O's, Symbol Match, Color Match, and Three Letter Memory) to generate four composite scores. Each testing module may contribute to more than one composite score. A detailed description of individual tests and composite scores are provided elsewhere. ${ }^{59,63,136}$ The four performance composite scores, consisting of verbal memory, visual memory, visual -motor processing speed and reaction time were used in this manuscript. Additionally, the total PCS score and the individual scores of symptoms were recorded. The PCS includes 22 items and is designed to quantify the severity of symptoms in the acute phase of recovery after concussion, using a 7 point Likert scale. For any particular symptom, the scale ranges from 0 (no symptom) to 6 (severe), and the total PCS score is calculated by adding the scores obtained for the 22 items.

The PCS has been evaluated for its psychometric properties, clinical interpretation and

normative scores. ${ }^{18,138,141}$ The time points considered for this report were the ImPACT tests that were closest to the start of vestibular physical therapy and the closest point to discharge from vestibular physical therapy, both within 30 days of the balance assessment. 


\subsubsection{Statistical analysis}

Descriptive statistics were used to describe the participants' demographic characteristics, time between concussion and ImPACT testing, and time between concussion and the start of vestibular physical therapy. In order to examine if the participants had more prevalent "vestibular/ balance" symptoms, a paired t-test was used to examine if the patients had higher symptom scores in the "vestibular" symptoms (as detailed below) compared to other symptoms around the start of vestibular physical therapy. A paired t-test was also used to examine if there was a difference in change scores between the vestibular symptoms and other symptoms over the course of vestibular physical therapy.

A vestibular/ balance subset of symptoms that included dizziness, nausea, balance problems, headache, sensitivity to light and sensitivity to sound was considered for correlation analysis with measures administered at the vestibular physical therapy.

Correlation analysis was performed to examine the relationship between the measures of dizziness severity rating, ABC, DHI, DGI, FGA, gait speed, TUG, FTSTS and the five composite scores of ImPACT at the starting point of vestibular physical therapy. The normality of the distribution was examined. The ABC, DHI, FGA, GS, TUG, FTSTS, and conditions $2,4,5,6$, of SOT were normally distributed. For the variables in ImPACT, all variables were normally distributed except reaction time. The natural log transformation for the reaction time was used throughout the analysis. For the variables that were not normally distributed (dizziness rating, SOT composite score and condition $1 \& 3$ of SOT), Spearman- rho correlation analysis was performed for the original score. A total of $70 \mathrm{c}$ orrelation analyses were conducted. To account for multiple comparisons, the False Discovery Rate (FDR) method described by 
Benjamini and Hochberg was used. ${ }^{317}$ False discovery rate is the expected proportion of erroneous rejections among all rejections.

Correlation analysis was also performed to examine the relationship between change in vestibular/ balance measures and change in ImPACT measures over the course of vestibular physical therapy. For the correlation in the change scores, Spearman- rho correlation coefficient was used for all analyses.

\subsection{RESULTS}

\subsubsection{Analysis at rehab start}

Ninety-three participants (59 F/34 M) out of 114 had received ImPACT testing around the time when they were initially seen for vestibular physical therapy after concussion. Of the participants included in this analysis, $65 \%(\mathrm{n}=60)$ were children (i.e. $<18$ y.o). Of the 60 children included in this analysis, the concussive injury was sport -related in 29 participants, while another 29 participants endured non-sport related concussion. The cause of concussion was unidentified for two participants. For the adult participants $(\mathrm{n}=33)$, only eight participants suffered a sportrelated concussion while the majority of the adult participants $(n=25)$ endured non- sport related concussion (e.g. fall, motor vehicle accident, assault).

The median age for the participants included in the analysis was 16 years (range: $11-54$ ).

They were seen at a median of 50 days after concussion (range: 4 -2566) days. The ImPACT testing was administered within 29 days of the start of their vestibular physical therapy $(\mathrm{M}=2.46$ days, $\mathrm{SD}=11.2$ da ys, Median $=2, \mathrm{r}$ ange 58 ( 29 days before-29 days after $)$. 
Participants were more symptomatic in the "vestibular" symptoms $(\mathrm{M}=2.6)$ compared to other symptoms $\left(\mathrm{M}=2.0, \mathrm{t}_{92}=-6.0, \mathrm{p}<.001\right)$.

\subsubsection{Relationship between clinical balance and neurocogntive measures}

Using the FDR method to adjust for multiplicity of analyses, 33 ou t of 70 correlations were significant (Table 6-1).

Greater total PCS scores were significantly related to greater impairment in all of the selfreport measures, worse performance in 3 of the 4 clinical performance measures, and greater sway during only one of the SOT conditions (fixed surface with eyes closed). The strongest correlation for the PCS was with the self-reported dizziness handicap inventory score $(r=0.49)$.

Of the neurocognitive measures of ImPACT, the visual memory score was correlated most times with the self-report and performance measures. Lower (i.e. worse) visual memory scores were associated with worse impairments in the self-report and balance measures.

Furthermore, lower visual memory scores were associated with greater sway in 5 of the 6 SOT conditions. In particular, visual memory performance was most strongly related to postural sway on a solid surface with eyes closed.

Reaction time, verbal memory, and processing speed were related to the self-report and clinical performance measures to a slightly lesser degree than visual memory. In all cases, worse neurocognitive performance was associated with the expected deficits in self-report measures and balance performance. 
Table 6-1: Relationship between ImPACT composite scores and measures administered at the start of vestibular physical therapy

\begin{tabular}{|c|c|c|c|c|c|}
\hline & Visual memory & Reaction time & Verbal memory & Processing speed & Total PCS score \\
\hline \multicolumn{6}{|l|}{ Self-Report } \\
\hline $\begin{array}{l}\text { Dizziness rating } t ~(n= \\
90)\end{array}$ & $r_{s}=-.15, p=.159$ & $r_{s}=.09, p=.425$ & $r_{s=}=.04, p=.684$ & $r_{s}=-.02, p=.889$ & $r_{s}=.24^{*}, p=.021$ \\
\hline$A B C(n=90)$ & $r=.37^{*}, p=<001$ & $r=-.36^{*}, p=<001$ & $r=.25^{*}, p=.020$ & $r=.27^{*}, p=.010$ & $r=-.39^{*}, p=<001$ \\
\hline $\mathrm{DHI}(\mathrm{n}=90)$ & $r=-.28^{*}, p=.008$ & $r=.27^{*}, p=.011$ & $r=-.22, p=.041$ & $r=-.21, p=.048$ & $r=.49 *, p=<001$ \\
\hline \multicolumn{6}{|l|}{ Clinical Performance } \\
\hline FGA $(n=82)$ & $r=.43^{*}, p=<001$ & $r=-.40 *, p=<001$ & $r=.40^{*}, p=<001$ & $r=.31^{*}, p=.004$ & $r=-.38^{*}, p=<001$ \\
\hline $\mathrm{GS}(\mathrm{n}=84)$ & $r=.31^{*}, p=.004$ & $r=-.17, p=.114$ & $r=.33^{*}, p=.002$ & $r=.20, p=.066$ & $r=-.32^{*}, p=.003$ \\
\hline TUG $(n=71)$ & $r=-.24, p=.045$ & $r=.31^{*}, p=.009$ & $r=-.19, p=.105$ & $r=-.21, p=.081$ & $r=.14, p=.239$ \\
\hline FTSTS $(n=69)$ & $r=-.41^{*}, p=<001$ & $r=.45^{*}, p=<001$ & $r=-.38^{*}, p=.001$ & $r=-.29 *, p=.014$ & $r=.38^{*}, p=.001$ \\
\hline \multicolumn{6}{|l|}{$\begin{array}{l}\text { Sensory Organization } \\
\text { Test (SOT) }\end{array}$} \\
\hline SOT composite† $(n=49)$ & $r_{s}=.32, p=.024$ & $r_{s}=-.18, p=.221$ & $r_{s}=.11, p=.437$ & $r_{s}=.20, p=.171$ & $r_{s}=-.26, p=.074$ \\
\hline SOT $1 \dagger(n=48)$ & $r_{s}=.23, p=.118$ & $r_{s}=-.11, p=.451$ & $r_{s}=.19, p=.190$ & $r_{s}=.08, p=.585$ & $r_{s}=.004, p=.977$ \\
\hline SOT $2(n=48)$ & $r=.53^{*}, .000$ & $r=-.46^{*}, p=.001$ & $r=.46^{*}, \mathrm{p}=.001$ & $r=.37^{*}, \mathrm{p}=.011$ & $r=-.36^{*} p=.012$ \\
\hline SOT 3† ( $n=48)$ & $r_{s}=.47^{*}, p=.001$ & $r_{s}=-.40 *, p=.005$ & $r_{s}=.30, p=.036$ & $r_{s}=.26, p=.070$ & $r_{s}=-.31, p=.031$ \\
\hline SOT $4(n=48)$ & $r=.40^{*}, p=.005$ & $r=-.26, p=.072$ & $r=.30, p=.040$ & $r=.30, p=.039$ & $r=-.18, p=.222$ \\
\hline SOT $5(n=48)$ & $r=.37^{*}, p=.010$ & $r=-.24, p=.105$ & $r=.27, p=.065$ & $r=.15, p=.309$ & $r=-.23, p=.122$ \\
\hline SOT $6(n=48)$ & $r=.33^{*}, p=.021$ & $r=-.16, p=.266$ & $r=.16, p=.288$ & $r=.12, p=.405$ & $r=-.22, p=.131$ \\
\hline
\end{tabular}

$\mathrm{n}=$ Number of participants, $\mathrm{ABC}=$ Activities-specific Balance Confidence scale, DHI = Dizziness Handicap Inventory, FGA $=$ Functional Gait Assessment, TUG $=$ Timed "Up \& Go", FTSTS $=$ Five Times Sit To Stand, SOT $=$ Sensory Organization Test, $r=$ Pearson product-moment correlation coefficient, $r_{s}$ Spearman's rank correlation coefficient 
For the measures where total PCS score was significantly related to self-report and clinical performance measures, a secondary analysis was conducted to examine if the vestibular subset of symptoms related better to these measures than the total symptom score. The results revealed that the total symptom score relates better to the DHI, GS, FTSTS and SOT compared to the "vestibular/ balance "subset that included dizziness, nausea, balance problems, headache, sensitivity to light and sensitivity to sound. However, the "vestibular/ balance "subset better relates to the $\mathrm{ABC}$ and dizziness rating compared to the total symptom score (Table 6-2).

Table 6-2: The relationship between outcome measures administered at the start of vestibular rehabilitation, total symptom score and "vestibular/ balance” subset of symptoms

\begin{tabular}{|c|c|c|}
\hline Outcome measure $(\mathrm{n})$ & Total symptom & Vestibularl balance \\
\hline $\begin{array}{c}\text { Dizziness rating } \\
(90)\end{array}$ & $r_{s=.243, \mathrm{p}=.021}$ & $R=-.453, \mathrm{p}<001$ \\
\hline $\begin{array}{c}\text { ABC } \\
(91)\end{array}$ & $r=-.393, \mathrm{p}<001$ & $r=.485, \mathrm{p}<001$ \\
\hline DHI & & $R=-.368, \mathrm{p}=.001$ \\
\hline F1) & $r=.494, \mathrm{p}<001$ & $R=-.294, \mathrm{p}=.007$ \\
\hline FGA & $r=-.382, \mathrm{p}<001$ & $r=.327, \mathrm{p}=.006$ \\
\hline GS & $r=-.316, \mathrm{p}=.003$ & \\
\hline FTSTS & $r=.381, \mathrm{p}=.001$ & \\
\hline (69) & & \\
\hline
\end{tabular}

$\mathrm{n}=$ Number of participants, $\mathrm{ABC}=$ Activities-specific Balance Confidence scale, DHI = Dizziness Handicap Inventory, FGA $=$ Functional Gait Assessment, FTSTS $=$ Five Times Sit To Stand, GS $=$ Gait Speed, $r=$ Pearson product-moment correlation coefficient, $r_{\mathrm{s}}$ Spearman's rank correlation coefficient

\subsubsection{Analysis of change scores}

Of the 93 participants who had received ImPACT testing around the time of initial assessment of vestibular physical therapy, 22 participants did not continue in vestibular therapy after the initial evaluation, and therefore, they were excluded from this analysis. Seventy-one participated in vestibular physical therapy program. However, 29 of the subjects did not have an ImPACT 
follow up during the time were they were seen for vestibular physical therapy (i.e. no change scores for ImPACT). Therefore, 39 participants $(29 \mathrm{~F} / 10 \mathrm{M})$ received two ImPACT tests; one around the start of vestibular physical therapy and one around the time of discharge from vestibular physical therapy, and qualified for the analysis of relationship in change scores.

There was no significant difference between the group that is included in the analysis ( $\mathrm{n}$ $=39$ ) and the group who did not qualify for this analysis $(\mathrm{n}=54)$, in time since concussion (Mann- Whitney $\mathrm{U}=809, \mathrm{p}=.144$ ), age (Mann- Whitney $\mathrm{U}=937, \mathrm{p}=.787$ ), or treatment duration (Mann- Whitney $\mathrm{U}=341, \mathrm{p}=.096$ ). However, the group included in the analysis of change scores reported higher dizziness severity and exhibited significantly worse performance on the DGI, FGA and SOT recorded at the start of vestibular physical therapy, compared with the group that were not included in this analysis at the time of initial evaluation in vestibular physical therapy (Table 6-3).

Of the 39 participants included in this analysis, $67 \%(n=26)$ were children (i.e. $<18$ y.o). The median age for the participants included in the analysis was 16.1 years (range: 11 - 47).

They were seen after a median of 46 days after concussion (range: 4 -168). The ImPACT testing was administered within 29 days around the start of their vestibular physical therapy (M $=2$ days, $\mathrm{SD}=8.1$ days, Median $=1$, range $(10$ before -23 after $)$. The ImPACT testing closest to the discharge from vestibular physical therapy was administered within 29 da ys around the discharge from vestibular physical therapy $(\mathrm{M}=0$ (i.e. ImPACT testing and discharge from therapy were on the same day), $\mathrm{SD}=10.9$ days, Median $=$ same day, range: $(26$ before -25 after). 
Table 6-3: Difference in the initial outcome measures between subjects not included in change analysis $(n=54)$ and subjects included in change analysis $(n=39)$

\begin{tabular}{|c|c|c|c|c|c|}
\hline $\begin{array}{c}\text { Outcome } \\
\text { measure at } \\
\text { the time of } \\
\text { initial } \\
\text { evaluation }\end{array}$ & \multicolumn{2}{|c|}{$\begin{array}{l}\text { Participants not in change } \\
\text { analysis }\end{array}$} & \multicolumn{2}{|c|}{$\begin{array}{l}\text { Participants in change } \\
\text { analysis }\end{array}$} & t-test,p value \\
\hline \multicolumn{6}{|c|}{ ImPACT composite } \\
\hline & mean (SD) & $\mathbf{n}$ & mean (SD) & $\mathbf{N}$ & \\
\hline $\begin{array}{c}\text { Total PCS } \\
\text { score }\end{array}$ & $45(32)$ & 54 & $51(24)$ & 39 & $t_{91}=-1.1, .26$ \\
\hline $\begin{array}{l}\text { Verbal } \\
\text { memory }\end{array}$ & $79(14)$ & 54 & $75(17)$ & 39 & $\mathrm{t}_{91}=1.2, .23$ \\
\hline $\begin{array}{l}\text { Visual } \\
\text { memory }\end{array}$ & $66(16)$ & 54 & $61(16)$ & 39 & $t_{91}=1.7, .09$ \\
\hline $\begin{array}{c}\text { Processing } \\
\text { speed }\end{array}$ & $30.5(10.2)$ & 54 & $30.0(11.3)$ & 39 & $t_{91}=.23, .82$ \\
\hline Reaction time & $.70(.27)$ & 54 & $.73(24)$ & 39 & $t_{91}=-.6, .51$ \\
\hline \multicolumn{6}{|c|}{ Vestibular rehab measures } \\
\hline & mean (SD) & $\mathbf{n}$ & mean (SD) & $\mathbf{n}$ & \\
\hline $\begin{array}{l}\text { Dizziness } \\
\text { rating }\end{array}$ & $14(18)$ & 53 & $30(22)$ & 31 & $t_{82}=-3.5, .001^{*}$ \\
\hline ABC & $71(28)$ & 52 & $62(25)$ & 32 & $t_{82}=1.4, .16$ \\
\hline DHI & $42(21)$ & 52 & $49(18)$ & 38 & $t_{83}=-1.8, .08$ \\
\hline DGI & $22(2)$ & 44 & $20(4)$ & 23 & $t_{27.7}=2.8, .009^{*}$ \\
\hline FGA & $26(4)$ & 44 & $23(6)$ & 23 & $t_{31.3}=2.6, .02^{*}$ \\
\hline GS (m/sec) & $1.14(.26)$ & 46 & $1.03(.22)$ & 22 & $t_{66}=1.7, .06$ \\
\hline TUG (sec) & $8.3(1.7)$ & 33 & $9.4(2.7)$ & 20 & $t_{27.6}=-1.6, .11$ \\
\hline FTSTS (sec) & $9.7(3.5)$ & 37 & $10.8(3.8)$ & 18 & $t_{54}=-1.1, .28$ \\
\hline SOT & $65(12)$ & 24 & $45(23)$ & 13 & $t_{15.9}=3.01, .008^{*}$ \\
\hline
\end{tabular}

The change in self-report and clinical performance measures was calculated over an average period of 50 days, $(\mathrm{SD}=44$ days) and the change in ImPACT was calculated over an average period of 49 da ys $(\mathrm{SD}=43$ days). A paired t-test revealed that this group of 39 participants who were included in this analysis exhibited a greater change in the vestibular/ balance symptoms subset $(\mathrm{M}=-1.5, \mathrm{SD}=1.0)$ compared to other symptoms $(\mathrm{M}=-0.9, \mathrm{SD}=0.9)$ $=$ over the course of vestibular physical therapy $\left(t_{38}=-6.1, p<.001\right)$. 
The results of analysis of relationship between change in ImPACT composite scores and change in self-report and clinical performance measures revealed that no significant relationship was found after adjusting for multiple comparisons using the FDR method (Table 6-4).

Table 6-4: Relationship between change in Vestibular/ balance measures and change in imPACT composites and the total symptom score

\begin{tabular}{|c|c|c|c|c|c|}
\hline $\begin{array}{c}\text { Change score } \\
\text { (n) }\end{array}$ & Verbal memory & Visual memory & Processing speed & Reaction time & $\begin{array}{c}\text { Total symptom } \\
\text { score }\end{array}$ \\
\hline $\begin{array}{c}\text { Dizziness rating } \\
(31)\end{array}$ & $r_{s}=-.153, p=.413$ & $r_{s}=-.052, p=.781$ & $r_{s}=.134, p=.473$ & $r_{s}=-.079, p=.674$ & $r_{s}=-.074, p=.694$ \\
\hline $\begin{array}{l}A B C \\
(32)\end{array}$ & $r_{s}=.036, p=.847$ & $r_{s}=.107, p=.559$ & $r_{s}=.191, . p=296$ & $r_{s}=-.199, p=.276$ & $r_{s}=-.333, p=.062$ \\
\hline $\begin{array}{l}\text { DHI } \\
(33) \\
\end{array}$ & $r_{s}=-.275, p=.122$ & $r_{s}=-.222, \quad p=.122$ & $r_{s}=-.366, p=.036$ & $r_{s}=.166, p=.356$ & $r_{s}=.345, p=.049$ \\
\hline $\begin{array}{l}\text { FGA } \\
(23)\end{array}$ & $r_{s}=.327, p=.128$ & $r_{s}=.201, p=.358$ & $r_{s}=.592, p=.003$ & $r_{s}=-.335, p=.118$ & $r_{s}=-.070, p=.750$ \\
\hline $\begin{array}{l}\text { GS } \\
(22)\end{array}$ & $r_{s}=.282, p=.204$ & $r_{s}=.222, p=.321$ & $r_{s}=.377, p=.084$ & $r_{s}=-.232, p=.299$ & $r_{s}=.064, p=.776$ \\
\hline $\begin{array}{l}\text { TUG } \\
(20) \\
\end{array}$ & $r_{s}=-.438, p=.053$ & $r_{s}=-.008, p=.972$ & $r_{s}=-.366, p=.112$ & $r_{s}=.059, p=803$ & $r_{s}=-.197, p=.406$ \\
\hline $\begin{array}{c}\text { FTSTS } \\
(18)\end{array}$ & $r_{s}=-.265, p=.287$ & $r_{s}=-.232, p=.354$ & $r_{s}=-.292, p=.240$ & $r_{s}=.688, p=.002$ & $r_{\mathrm{s}}=.171, p=.499$ \\
\hline
\end{tabular}

The analysis of whether a change in the "vestibular" symptoms of PCS relates better to the change in the vestibular measures than total symptom score revealed that no differences were found between the change in vestibular symptoms when compared to the change in the total symptom score. (Table 6-5) 
Table 6-5: Relationship between change in symptom scores and change in measures administered over the course of vestibular rehabilitation

\begin{tabular}{|c|c|c|}
\hline $\begin{array}{c}\text { Change in outcome measure } \\
(\mathbf{n})\end{array}$ & $\begin{array}{c}\text { Change in total symptom } \\
\text { score }\end{array}$ & $\begin{array}{c}\text { Change in vestibular } \\
\text { symptoms }\end{array}$ \\
\hline $\begin{array}{c}\text { Dizziness rating } \\
(\mathbf{3 1})\end{array}$ & $\mathrm{r}_{\mathrm{s}}=-.074, \mathrm{p}=.69$ & $\mathrm{r}_{\mathrm{s}}=.026, \mathrm{p}=.89$ \\
\hline $\begin{array}{c}\text { ABC } \\
(\mathbf{3 2})\end{array}$ & $\mathrm{r}_{\mathrm{s}}=-.333, \mathrm{p}=.06$ & $\mathrm{r}_{\mathrm{s}}=-.348, \mathrm{p}=.05$ \\
\hline $\begin{array}{c}\text { DHI } \\
(\mathbf{3 3 )}\end{array}$ & $\mathrm{r}_{\mathrm{s}}=.345^{*}, \mathrm{p}=.049$ & $\mathrm{r}_{\mathrm{s}}=.307, \mathrm{p}=.08$ \\
\hline $\begin{array}{c}\text { FGA } \\
(\mathbf{2 3 )}\end{array}$ & $\mathrm{r}_{\mathrm{s}}=-.070, \mathrm{p}=.75$ & $\mathrm{r}_{\mathrm{s}}=-.072, \mathrm{p}=.75$ \\
\hline $\begin{array}{c}\text { GS } \\
(\mathbf{2 2})\end{array}$ & $\mathrm{r}_{\mathrm{s}}=.064, \mathrm{p}=.77$ & $\mathrm{r}_{\mathrm{s}}=-.057, \mathrm{p}=.80$ \\
\hline $\begin{array}{c}\text { TUG } \\
(\mathbf{2 0 )}\end{array}$ & $\mathrm{r}_{\mathrm{s}}=-.197, \mathrm{p}=.41$ & $\mathrm{r}_{\mathrm{s}}=-.136, \mathrm{p}=.57$ \\
\hline $\begin{array}{c}\text { FTS } \\
(\mathbf{1 8 )}\end{array}$ & $\mathrm{r}_{\mathrm{s}}=.171, \mathrm{p}=.49$ & $\mathrm{r}_{\mathrm{s}}=.386, p=.11$ \\
\hline
\end{tabular}

$\mathrm{n}=$ Number of participants, $\mathrm{ABC}=$ Activities-specific Balance Confidence scale, DHI $=$ Dizziness Handicap Inventory, FGA $=$ Functional Gait Assessment, GS = Gait Speed, TUG = Timed "Up \& Go", FTSTS = Five Times Sit To Stand, $r_{s}=$ Spearman's rank correlation coefficient

\subsection{DISCUSSION}

A number of significant correlations (33/70) were found between the ImPACT performance scores and measures administered at the time of start of vestibular physical therapy. However, no significant relationships were found between the change in ImPACT composites and the change in gait/ balance measures. Although all performance composite scores in ImPACT exhibited some significant correlations with measures administered at the start of vestibular physical therapy, it was noted that correlations were centered on visual memory compared to reaction time, verbal memory and processing speed evidenced by more number of significant relationships found with visual memory. Additionally, although FGA, FTSTS and Condition 2 of 
SOT were significantly correlated to all ImPACT composite scores, the correlation coefficients were higher (i.e. moderate) for visual memory and reaction time compared with correlations exhibited for verbal memory and processing speed. Visual memory is a composite score that is extracted from the Design Memory test and the X's and O's test in ImPACT. The vestibular subset of symptoms seems to relate better to the self report measures of dizziness severity rating and $\mathrm{ABC}$. However, the vestibular subset of symptoms in this manuscript did not exhibit stronger relationship to the clinical balance measures compared to the total symptom score.

The comparison between the results of this manuscript with the results of previous research that studied the correlation between measures after concussion is limited. According to our knowledge, there were no previous research studies that have examined the relationship between ImPACT composite scores and the balance outcomes used in this study. A previous study suggested a relationship between self- report symptoms related to neurocognition (i.e. difficulty remembering, difficulty concentration, and feeling mentally foggy) and neurocogntive testing performance. ${ }^{21}$ Also, a relationship was supported between self- reported symptoms related to balance (i.e. dizziness and balance problems) and balance performance. However, the evidence is less clear when the comparison is made between neurocognitive testing and balance performance. Broglio et al. examined if the symptoms of "feeling mentally foggy", "difficulty concentrating", and "difficulty remembering" are associated with decreased cognitive performance (i.e. ImPACT composite scores). The results revealed a significant correlation between "feeling mentally foggy" and reaction time $\left(\mathrm{r}_{\mathrm{s}}=0.36\right)$, between "difficulty concentrating" and verbal memory $\left(\mathrm{r}_{\mathrm{s}}=-0.41\right)$, and between "difficulty remembering" and change in verbal memory and reaction time $\left(r_{s}=0.48\right.$ and 0.36 , respectively). ${ }^{21}$ 
The absence of a relationship between the change scores in measures administered during vestibular physical therapy may indicate that the recovery of neurocognitive domains and gait/ balance deficits are different. Moreover, over the period when change scores were calculated, patients received a customized intervention based on the deficits found at the initial vestibular evaluation and they were discharged based on the improvement in vestibular outcomes at the time of discharge. Consequently, this specificity of intervention to vestibular impairments may have contributed to the lack of relationship between the change in ImPACT composite scores and change in vestibular symptoms. The significant difference found between change in "vestibular" symptoms $(\mathrm{M}=-1.5, \mathrm{SD}=1.0)$ compared to other symptoms $(\mathrm{M}=-0.9, \mathrm{SD}=0.9)$, $\left(\mathrm{t}_{38}=-6.1, \mathrm{p}<.001\right)$ may support the notion that the vestibular/ balance symptoms may have changed differently attributed in part to the vestibular physical therapy exercises. Therefore, this may have altered the relationship with total symptom score.

Additionally, the absence of relationships found in this sample may be explained by reasons related to the methodology and analysis used in the current manuscript; the analysis of change relationships may have been underpowered due to the following factors: 1) Small number of participants included in each analysis; although 39 pa rticipants qualified for the change analysis, the actual numbers in each analysis ranged between 18 and 33, 2). Since we have also used the FDR to control for the multiplicity of analyses, we may have further limited our ability to find significant results.

Although the composite scores of visual memory and reaction time seem to better relate to the initial measures of vestibular physical therapy, a different pattern is observed when the relationship is closely examined for the change scores. If the relationship in change scores is to be examined without adjustment for multiplicity of analysis (i.e. $\alpha=0.05$ ), the visual-motor 
processing speed is significantly correlated with the change in DHI and FGA (and SOT composite scores and Conditions 3, 5 and 6 not shown). This change in the trends of relationship may suggest the exercises provided in the vestibular physical therapy may have been beneficial to the cognitive domain of visual-motor processing speed.

The participants in this study were referred to vestibular physical therapy and they reported significantly worse symptoms in "vestibular "symptoms compared to other symptoms suggesting that the referral for vestibular physical therapy clinic was appropriate. Although this group of concussion patients can be labeled as vestibular patients, the deficits reported in neurocognitive performance testing (i.e. ImPACT) emphasize the universal effects of concussion even within this group of individuals with concussion that have complaints of vestibular symptoms beyond the third week after concussion.

The findings of this study re- emphasize the need for a comprehensive approach of concussion management that takes into account the effects of concussion on different areas of brain functioning and allow clinicians to make appropriate recommendations on return to play or work after concussion. ${ }^{103}$

Although this study aimed to examine the relationship between the neurocognitive and balance performance and recovery after concussion, the design of the study was limited; therefore, the results of this study are only preliminary and intended to guide future research directions. The participants included in this study were obtained from a clinical population of patients referred by one practice for vestibular physical therapy were the referral process was solely based on clinical judgment and is often made if the patients are not recovering concussion.

The majority of participants $[\mathrm{n}=85(91 \%)]$ were referred after three weeks of concussion, and therefore this sample does not represent the wide spectrum of individuals with 
concussion who will make full recovery by the third week post- concussion. The relationship between neurocognitive functioning and balance functioning may have been different if examined in the acute (i.e. $<3$ weeks) vs. chronic phase after concussion. Furthermore, the participants of this study are clearly experiencing "vestibular" symptoms compared to other symptoms, suggesting the referral to vestibular physical therapy was a sound clinical decision.

However, there might have been individuals with prolonged recovery that are managed in other adjacent interventions and were not referred to vestibular physical therapy. Consequently, the relationships found in this study could be different if examined in a larger group of individuals with prolonged recovery after concussion (with and without vestibular deficit).

In summary, the participants of this study represent a small percentage of individuals with concussion who are thought to benefit from vestibular physical therapy after balance/dizziness related symptoms that lasted beyond the normal window of recovery. Therefore, this sample does not represent the majority of individuals with concussion who achieve full recovery before the third week after injury.

Furthermore, due to the retrospective design of this manuscript, the time points considered for the correlation analyses were highly variable between participants and may have influenced the findings of this study. Although the time between vestibular measures and ImPACT testing was not significantly related to any of the measures; it may have affected the strength of correlation coefficients. A prospective design that allows concurrent neurocognitive and gait/ balance evaluation may provide a better estimation about the relationship between neurocogntive and gait/ balance functioning after concussion.

The results obtained from the analysis of relationship between change scores have additional limitations; the participants included in this analysis tend to be more impaired in the 
gait/ balance measures at the start of vestibular physical therapy and therefore, they may represent even a smaller group of individuals with prolonged balance deficits after concussion and does not represent the whole spectrum of individuals with prolonged balance deficits after concussion.

Furthermore, while being treated with vestibular physical therapy, some participants were treated with other interventions such as medications, an exertional physical therapy program, or had restricted work or school access in order to minimize mental and physical exertion and to expedite recovery. It is unclear if the different adjacent interventions may have affected the results found in this group. Without a control group, the role of adjacent interventions on the relationship between the recovery of different domains cannot be ruled out. However, the adjacent interventions provided for some participants in this study were reported in clinicians' notes, and were not systematically recorded in the medical charts making the examination of its role in the recovery process unattainable. A future prospective study design should consider the uniformity of interventions for all participants, and should allow examining the effects of adjacent interventions on the interrelationships between recovery of neurogognitive and gait/ balance outcomes after concussion.

The participants in this study endured sport-related and non-sport related concussions.

While the majority of concussion injuries in the adult participants were caused by nonsport injuries, the sport related concussions represent almost $50 \%$ of concussions in children participants (i.e. $<18$ y.o). Due to power related issues, we did not examine if the relationship would be different between sport-related and non-sport related injuries and we did not examine if the relationship is different between children and adults. 
Although we acknowledge the limitations in this study, we believe that this study can serve as a starting point to direct future research to examine the interrelationships between the recovery of different domains. Despite the limitations of this study, the results demonstrated that the relationships between gait/ balance measures appear to be centered on the visual memory and reaction time at referral. However, the change obtained in vestibular measures may appear to be centered on the visual-motor processing speed, and therefore future research should consider these domains when examining the relationship between neurocognitive functioning and gait/ functional balance testing for further research.

\subsection{CONCLUSION}

The significant relationships between the ImPACT neurocognitive performance scores and balance measures at the start of vestibular rehabilitation may reflect that similar levels of functioning exist across domains. Future areas of exploration will include assessment of changes in neurocognitive and balance function during vestibular rehabilitation to see if recovery of these measures have similar trajectories. 


\subsection{FUTURE CONSIDERATIONS AND FUTURE DIRECTIONS}

\subsection{FUTURE CONSIDERATIONS}

Based on the limitations we have encountered in the current dissertation, I have the following suggestions for future considerations. In the first aim, we aimed to provide normative data and to examine the reliability for clinical gait/ balance measures for healthy high school aged children.

We chose the participants from two private parochial schools in an urban location (one female only, and one male only) and the other school was a public co-educational school in a suburban school district. However, for future considerations, the schools should represent a wider spectrum of socioeconomic status of rural and public schools. A future study should include equal representation of students at all ages within our age range. Additionally we did not collect race/ ethnicity data. However, race/ ethnicity should be reported to provide an indication of how well participants match to the overall high school demographics of the USA. The participants in aim one included athletes and non a thletes, a future study can examine if the normative reference scores are different between athletes and non athletes. For the reliability analysis, we should examine the inter rater reliability of the BESS test as it is being scored clinically vs. videotaping.

When we examined the outcome of vestibular rehabilitation exercises, the majority of our samples were referred for vestibular physical therapy while being managed with other 
interventions. Unfortunately, there was no systematic reporting of other interventions and therefore, the relative contribution of vestibular rehabilitation was not determined. A future prospective study can examine the relative contribution of vestibular physical therapy and ensure the uniformity of interventions. Furthermore, the majority of our participants were referred for therapy beyond three weeks after concussion. A future study may consider if early referral affects the outcomes of vestibular rehabilitation vs. later referrals for vestibular therapy.

When we recorded the exercise prescription patterns, we were not able to determine the rationale for the exercise prescription; a future prospective study should examine the therapist rationale for the exercise prescription and the rational for exercise progression. A prospective design will also allow for less missing data.

Another suggestion for aim four is to design a prospective study in which the neurocognitive testing and the balance testing are being concurrently examined. A prospective study can also examine if the relationship between neurocognitive and balance recovery is different between individuals treated with vestibular physical therapy vs. individuals who are not being treated with vestibular physical therapy.

\subsection{FUTURE DIRECTIONS}

In order to provide a higher quality of evidence, I suggest the following design. Individuals with concussion who are being seen by a neuropsychologist within the first three days of concussion should be examined for both neurocognitive and balance deficits Time 1(t1). The results of this testing will place the patients into one of three categories 1) Individuals with no neurocogntive deficits and no ba lance deficits, 2) Individuals with neurocogntive deficits but no balance 
deficits, and 3) Individuals with balance deficits (compared to reference scores) or high balance symptom score (based on the "vestibular "subset of symptoms).

The individuals who did not have neuropsychological or balance impairments will not be eligible whereas the individuals in the other two categories could be recruited for the study, after the participants were enrolled in the study, the individuals who presented with neurocognitive but not a balance deficit can be managed with traditional concussion management for five weeks.

The individuals who presented with balance deficits and/ or balance symptoms can be allocated to one of the two study groups; the experimental group that includes a cu stomized physical therapy program that is provided for the individuals two visits every week for 5 weeks (based on the median number of treatment duration found in the second aim of the dissertation).

The control group will be managed by conventional concussion management approach for five weeks. After the fifth week, all participants will be examined for neurocognitive and balance testing.

An independent t- test is conducted to examine if the individuals with balance deficits (category 3) also exhibited worse neuropsychological performance compared with individuals with no balance deficits (category 2) immediately post concussion.

After the five weeks, the change scores in neuropsychological and balance measures is calculated $(\mathrm{t} 2-\mathrm{t} 1)$, an independent $\mathrm{t}$ - test is conducted to examine if the presence of the balance deficits is associated with worse neuropsychological recovery by examining if the recovery in ImPACT composite scores is different between the individuals with balance deficits (the control group of category 3) and the individuals with only neurocogntive deficits (category 2).

For individuals in category three (balance deficits), an independent t- test can be conducted to ensure the participants were not significantly different on a ny of the outcome 
measures at the time of group allocation. An independent $t$ - test is conducted to examine if the change scores are significantly different between the groups treated with vestibular PT when compared with the group of conventional concussion management (effect of vestibular PT).

After the change scores in neurocognitive measures and balance measures is calculated for all participants, a co rrelation analysis is conducted. The Fisher's $z$ test is conducted to examine if the correlation between neuropsychological and balance recovery is different between individuals treated with vestibular rehabilitation vs. individuals treated with conventional management. 


\section{BIBLIOGRAPHY}

1. Rutland-Brown $\mathrm{W}$, Langlois JA, Thomas KE, Xi YL. Incidence of traumatic brain injury in the United States, 2003. J. Head Trauma Rehabil. 2006;21:544-548.

2. Control CfD. http://www.cdc.gov/ncipc/pubres/TBI_in_US 04/TBI\%20Tables_2003.pdf. $\quad$ http://www.cdc.gov/ncipc/pubres/TBI in US 04/TBI\%20Tables 2003.pdf. Accessed 11/06/2009, 2009.

3. Kraus JF. Epidemiological features of brain injury in children: occurrence, children at risk, causes and manner of injury, severity and outcomes. In: S. B, M. M, eds. Traumatic head injury in children: Oxford University Press; 1995:22-39.

4. McCrory P, Collie A, Anderson V, Davis G. Can we manage sport related concussion in children the same as in adults? Br. J. Sports Med. 2004;38:516-519.

5. Kraus JF, Nourjah P. The epidemiology of mild, uncomplicated brain injury. J Trauma. 1988;28:1637-1643.

6. Annegers JF, Grabow JD, Kurland LT, Laws ER, Jr. The incidence, causes, and secular trends of head trauma in Olmsted County, Minnesota, 1935-1974. Neurology. 1980;30:912-919.

7. Cassidy JD, Carroll LJ, Peloso PM, et al. Incidence, risk factors and prevention of mild traumatic brain injury: results of the WHO Collaborating Centre Task Force on Mild Traumatic Brain Injury. J Rehabil Med. 2004:28-60.

8. Langlois JA, Rutland-Brown W, Wald MM. The epidemiology and impact of traumatic brain injury: a brief overview. J. Head Trauma Rehabil. 2006;21:375-378.

9. Belanger $\mathrm{HG}$, Vanderploeg RD. The neuropsychological impact of sports-related concussion: a meta-analysis. J. Int. Neuropsychol. Soc. 2005;11:345-357.

10. Bleiberg J, Cernich AN, Cameron K, et al. Duration of cognitive impairment after sports concussion. Neurosurgery. 2004;54:1073-1078.

11. Catale $\mathrm{C}$, Marique $\mathrm{P}$, Closset $\mathrm{A}$, Meulemans $\mathrm{T}$. Attentional and executive functioning following mild traumatic brain injury in children using the Test for Attentional Performance (TAP) battery. J Clin Exp Neuropsychol. 2009;31:331-338.

12. Iverson GL, Brooks BL, Collins MW, Lovell MR. Tracking neuropsychological recovery following concussion in sport. Brain Inj. 2006;20:245-252.

13. Gagnon I, Forget R, Sullivan SJ, Friedman D. Motor performance following a mild traumatic brain injury in children: an exploratory study. Brain Inj. 1998;12:843-853.

14. Guskiewicz KM. Postural stability assessment following concussion: one piece of the puzzle. Clin J Sport Med. 2001;11:182-189. 
15. Catena RD, van Donkelaar P, Chou LS. Different gait tasks distinguish immediate vs. long-term effects of concussion on balance control. J. Neuroeng \& Rehabil. 2009;6.

16. Guskiewicz KM. Assessment of postural stability following sport-related concussion. . Curr. Sports med rep. 2003;2:24-30.

17. Randolph C, Millis S, Barr WB, et al. Concussion symptom inventory: an empirically derived scale for monitoring resolution of symptoms following sport-related concussion. Arch Clin Neuropsychol. 2009;24:219-229.

18. Lovell MR, Iverson GL, Collins MW, et al. Measurement of symptoms following sportsrelated concussion: reliability and normative data for the post-concussion scale. Appl. Neuropsych. 2006;13:166-174.

19. Broglio SP, Puetz TW. The effect of sport concussion on neurocognitive function, selfreport symptoms and postural control: A meta-analysis. Sports Med. 2008;38:53-67.

20. Broglio SP, Macciocchi SN, Ferrara MS. Sensitivity of the concussion assessment battery. Neurosurgery. 2007;60:1050-1057.

21. Broglio SP, Sosnoff JJ, Ferrara MS. The relationship of athlete-reported concussion symptoms and objective measures of neurocognitive function and postural control. Clin $J$ Sport Med. 2009;19:377-382.

22. McCrory P, Johnston K, Meeuwisse W, et al. Summary and agreement statement of the second international conference on concussion in sport, prague 2004. Phys Sportsmed. 2005;33:29-44.

23. Grindel SH, Lovell MR, Collins MW. The assessment of sport-related concussion: the evidence behind neuropsychological testing and management. Clin J Sport Med. 2001;11:134-143.

24. Maroon JC, Lovell MR, Norwig J, Podell K, Powell JW, Hartl R. Cerebral concussion in athletes: evaluation and neuropsychological testing. Neurosurgery. 2000;47:659-669.

25. Campbell M, Parry A. Balance disorder and traumatic brain injury: preliminary findings of a multi-factorial observational study. Brain Inj. 2005;19:1095-1104.

26. Gagnon I, Swaine B, Friedman D, et al. Children show decreased dynamic balance after mild traumatic brain injury. Arch. Phys. Med. Rehabil. 2004;85:444-452.

27. Basford JR, Chou LS, Kaufman KR, et al. An assessment of gait and balance deficits after traumatic brain injury. Arch. Phys. Med. Rehabil. 2003;84:343-349.

28. Kaufman KR, Brey RH, Chou LS, et al. Comparison of subjective and objective measurements of balance disorders following traumatic brain injury. Med Eng Phys. 2006;28:234-239.

29. Catena RD, van Donkelaar P, Chou LS. Altered balance control following concussion is better detected with an attention test during gait. Gait Posture. 2007;25:406-411.

30. Guskiewicz KM, Perrin DH, Gansneder BM. Effect of Mild Head Injury on P ostural Stability in Athletes. J Athl Train. 1996;31:300-306.

31. McCrea M, Guskiewicz KM, Marshall SW, et al. Acute effects and recovery time following concussion in collegiate football players: the NCAA Concussion Study. JAMA. 2003;290:2556-2563.

32. Johnston KM, Bloom GA, Ramsay J, et al. Current concepts in concussion rehabilitation. Curr. Sports med rep. 2004;3:316-323.

33. Gizzi M. Critical review: The efficacy of vestibular rehabilitation for patients with head trauma. J. Head Trauma Rehabil. 1995;10:60-77. 
34. Hoffer ME, Gottshall KR, Moore R, Balough BJ, Wester D. Characterizing and treating dizziness after mild head trauma. Otol. and Neurotol. 2004;25:135-138.

35. Comper P, Bisschop SM, Carnide N, Tricco A. A systematic review of treatments for mild traumatic brain injury. Brain Inj. 2005;19:863-880.

36. Elgmark Andersson E, Emanuelson I, Bjorklund R, Stalhammar DA. Mild traumatic brain injuries: the impact of early intervention on late sequelae. A randomized controlled trial. Acta Neurochirurgica. 2007;149:151-159.

37. Gordon CR, Levite $\mathrm{R}$, Joffe $\mathrm{V}$, et al. Is posttraumatic benign paroxysmal positional vertigo different from the idiopathic form? Arch Neurol. 2004;61:1590-1593.

38. Gottshall KR, Moore RJ, Hoffer ME, Gottshall KR, Moore RJ, Hoffer ME. Vestibular rehabilitation for migraine-associated dizziness. Int. Tinnit. J. 2005;11:81-84.

39. Herdman SJ. Treatment of vestibular disorders in traumatically brain-injured patients. $J$. Head Trauma Rehabil. 1990;5:63-76.

40. Kissick J, Johnston KM. Return to play after concussion: principles and practice. Clin $J$ Sport Med. 2005;15:426-431.

41. Friedman JM, Friedman JM. Post-traumatic vertigo. Med Health R I. 2004;87:296-300.

42. Davies RA, Luxon LM. Dizziness following head injury: a neuro-otological study. $J$ Neurol. 1995;242:222-230.

43. Gottshall K, Drake A, Gray N, et al. Objective vestibular tests as outcome measures in head injury patients. Laryngoscope. 2003;113:1746-1750.

44. Gurr B, Moffat N. Psychological consequences of vertigo and the effectiveness of vestibular rehabilitation for brain injury patients. Brain Inj. 2001;15:387-400.

45. Shumway-Cook A. Assessment and Management of the patient with Traumatic Brain Injury and Vestibular Dysfunction. In: Herdman SJ, ed. Vestibular Rehabilitation. Third Edition ed. Philadelphia: F.A. Davis company; 2007:444 - 457.

46. Belanger HG, Curtiss G, Demery JA, Lebowitz BK, Vanderploeg RD. Factors moderating neuropsychological outcomes following mild traumatic brain injury: a metaanalysis. J. Int. Neuropsychol. Soc. 2005;11:215-227.

47. Vanderploeg RD, Curtiss G, Belanger HG. Long-term neuropsychological outcomes following mild traumatic brain injury. J. Int. Neuropsychol. Soc. 2005;11:228-236.

48. Guskiewicz KM, Ross SE, Marshall SW. Postural Stability and Neuropsychological Deficits After Concussion in Collegiate Athletes. J Athl Train. 2001;36:263-273.

49. Lange RT, Iverson GL, Franzen MD. Neuropsychological functioning following complicated vs. uncomplicated mild traumatic brain injury. Brain Inj. 2009;23:83-91.

50. Fazio VC, Lovell MR, Pardini JE, Collins MW. The relation between post concussion symptoms and neurocognitive performance in concussed athletes. NeuroRehabilitation. 2007;22:207-216.

51. Kirkwood MW, Yeates KO, Taylor HG, Randolph C, McCrea M, Anderson VA. Management of pediatric mild traumatic brain injury: a neuropsychological review from injury through recovery. Clin. Neuropsych. 2008;22:769-800.

52. Carroll LJ, Cassidy JD, Holm L, Kraus J, Coronado VG. Methodological issues and research recommendations for mild traumatic brain injury: the WHO Collaborating Centre Task Force on Mild Traumatic Brain Injury. J Rehabil Med. 2004:113-125.

53. Medicine ACoR. Definition of mild traumatic brain injury. J. Head Trauma Rehabil. 1993;8:86-87. 
54. Neurology AAo. Practice parameter: the management of concussion in sports (summary statement). Report of the Quality Standards Subcommittee. Neurology. 1997;48:581-585.

55. Pediatrics AAo. The management of minor closed head injury in children. Committee on Quality Improvement, American Academy of Pediatrics. Commission on Clinical Policies and Research, American Academy of Family Physicians. Pediatrics. 1999;104:1407-1415.

56. Surgeons CoN. Committee on head injury nomenclature: Glossary of head injury. Clin. Neurosurg. 1966;12:386-384.

57. Collins MW, Hawn KL. The clinical management of sports concussion. Curr. Sports med rep. 2002;1:12-22.

58. Johnston KM, McCrory P, Mohtadi NG, Meeuwisse W. Evidence-Based review of sportrelated concussion: clinical science. Clin J Sport Med. 2001;11:150-159.

59. Schatz P, Pardini JE, Lovell MR, Collins MW, Podell K. Sensitivity and specificity of the ImPACT Test Battery for concussion in athletes. Arch Clin Neuropsychol. 2006;21:9199.

60. Reddy CC, Collins MW, Gioia GA. Adolescent sports concussion. Phys Med Rehabil Clin N Am. 2008;19:247-269.

61. Lovell MR, Iverson GL, Collins MW, McKeag D, Maroon JC. Does loss of consciousness predict neuropsychological decrements after concussion? Clin J Sport Med. 1999;9:193-198.

62. Collins MW, Iverson GL, Lovell MR, McKeag DB, Norwig J, Maroon J. On-field predictors of neuropsychological and symptom deficit following sports-related concussion. Clin J Sport Med. 2003;13:222-229.

63. Lovell MR, Collins MW, Iverson GL, Johnston KM, Bradley JP. Grade 1 or "ding" concussions in high school athletes. Am. J. Sports Med. 2004;32:47-54.

64. Field M, Collins MW, Lovell MR, Maroon J. Does age play a role in recovery from sports-related concussion? A comparison of high school and collegiate athletes. $J$ Pediatr. 2003;142:546-553.

65. Lovell MR, Collins MW, Iverson GL, et al. Recovery from mild concussion in high school athletes. J. Neurosurg. 2003;98:296-301.

66. Sim A, Terryberry-Spohr L, Wilson KR. Prolonged recovery of memory functioning after mild traumatic brain injury in adolescent athletes. J. Neurosurg. 2008;108:511-516.

67. Covassin T, Schatz P, Swanik CB. Sex differences in neuropsychological function and post-concussion symptoms of concussed collegiate athletes. Neurosurgery. 2007;61:345350 .

68. Broshek DK, Kaushik T, Freeman JR, Erlanger D, Webbe F, Barth JT. Sex differences in outcome following sports-related concussion. J. Neurosurg. 2005;102:856-863.

69. Colvin AC, Mullen J, Lovell MR, West RV, Collins MW, Groh M. The role of concussion history and gender in recovery from soccer-related concussion. Am. J. Sports Med. 2009;37:1699-1704.

70. McCrory P, Meeuwisse W, Johnston $\mathrm{K}$, et al. Consensus statement on Concussion in Sport 3rd International Conference on Concussion in Sport held in Zurich, November 2008. Clin J Sport Med. 2009;19:185-200.

71. Cohen JS, Gioia G, Atabaki S, Teach SJ. Sports-related concussions in pediatrics. Curr. Opin. Pediatr. 2009;21:288-293. 
72. Akhavan A, Flores C, Green S, et al. Clinical inquiries. How should we follow athletes after a concussion? J. Fam. Pract. 2005;54:902-904.

73. Thurman DJ. The Epidemiology and economics of head trauma. In: Miller L, Ayes R, eds. Head Trauma: Basic, Preclinical, and Clinical Directions. New York: John Wiley and Sons; 2001:327-347.

74. Wade DT, King NS, Wenden FJ, Crawford S, Caldwell FE. Routine follow up after head injury: a second randomised controlled trial. J Neurol Neurosurg Psychiatry. 1998;65:177-183.

75. Sports-related recurrent brain injuries--United States. MMWR Morb Mortal Wkly Rep. 1997;46:224-227.

76. Macciocchi SN, Barth JT, Alves W, Rimel RW, Jane JA. Neuropsychological functioning and recovery after mild head injury in collegiate athletes. Neurosurgery. 1996;39:510-514.

77. McCrea M, Kelly JP, Randolph C, et al. Standardized assessment of concussion (SAC): on-site mental status evaluation of the athlete. J. Head Trauma Rehabil. 1998;13:27-35.

78. Goldberg LD, Dimeff RJ, Goldberg LD, Dimeff RJ. Sideline management of sportrelated concussions. Sports med. 2006;14:199-205.

79. Powell JW, Barber-Foss KD. Traumatic brain injury in high school athletes. JAMA. 1999;282:958-963.

80. Collins MW, Grindel SH, Lovell MR, et al. Relationship between concussion and neuropsychological performance in college football players. JAMA. 1999;282:964-970.

81. Koh JO, Cassidy JD, Watkinson EJ. Incidence of concussion in contact sports: a systematic review of the evidence. Brain Inj. 2003;17:901-917.

82. Lee MA. Adolescent concussions--management recommendations: a practical approach. Conn. Med. 2006;70:377-380.

83. McCrea M, Hammeke T, Olsen G, Leo P, Guskiewicz K. Unreported concussion in high school football players: implications for prevention. Clin J Sport Med. 2004;14:13-17.

84. Guskiewicz KM, Bruce SL, Cantu RC, et al. National Athletic Trainers' Association Position Statement: Management of Sport-Related Concussion. $J$ Athl Train. 2004;39:280-297.

85. Williamson IJ, Goodman D. Converging evidence for the under-reporting of concussions in youth ice hockey. Br. J. Sports Med. 2006;40:128-132.

86. Sosin DM, Sniezek JE, Thurman DJ. Incidence of mild and moderate brain injury in the United States, 1991. Brain Inj. 1996;10:47-54.

87. Bazarian JJ, McClung J, Shah MN, Cheng YT, Flesher W, Kraus J. Mild traumatic brain injury in the United States, 1998--2000. Brain Inj. 2005;19:85-91.

88. Meehan WP, 3rd, Bachur RG. Sport-related concussion. Pediatrics. 2009;123:114-123.

89. Thurman DJ, Branche CM, Sniezek JE. The epidemiology of sports-related traumatic brain injuries in the United States: recent developments. J. Head Trauma Rehabil. 1998;13:1-8.

90. Kelly JP. Traumatic brain injury and concussion in sports. JAMA. 1999;282:989-991.

91. Finkelstein E, Corso P, Miller T. The Incidence and Economic Burden of Injuries in the United States. New York: Oxford University Press; 2006.

92. Findler M, Cantor J, Haddad L, Gordon W, Ashman T. The reliability and validity of the SF-36 health survey questionnaire for use with individuals with traumatic brain injury. Brain Inj. 2001;15:715-723. 
93. Rutherford WH, Merrett JD, McDonald JR. Symptoms at one year following concussion from minor head injuries. Injury. 1979;10:225-230.

94. Ganesalingam K, Yeates KO, Ginn MS, et al. Family burden and parental distress following mild traumatic brain injury in children and its relationship to post-concussive symptoms. J. Pediatr. Psychol. 2008;33:621-629.

95. McCrea M, Kelly JP, Randolph C, Cisler R, Berger L. Immediate neurocognitive effects of concussion. Neurosurgery. 2002;50:1032-1040.

96. Hinton-Bayre AD, Geffen GM, Geffen LB, McFarland KA, Friis P. Concussion in contact sports: reliable change indices of impairment and recovery. J Clin Exp Neuropsychol. 1999;21:70-86.

97. Maddocks D, Saling M. Neuropsychological deficits following concussion. Brain Inj. 1996;10:99-103.

98. Iverson GL. Complicated vs uncomplicated mild traumatic brain injury: acute neuropsychological outcome. Brain Inj. 2006;20:1335-1344.

99. Matser EJ, Kessels AG, Lezak MD, Jordan BD, Troost J. Neuropsychological impairment in amateur soccer players. JAMA. 1999;282:971-973.

100. McCrea M. Standardized mental status assessment of sports concussion. Clin J Sport Med. 2001;11:176-181.

101. Lovell MR, Collins MW. Neuropsychological assessment of the college football player. J. Head Trauma Rehabil. 1998;13:9-26.

102. Majerske CW, Mihalik JP, Ren D, et al. Concussion in sports: postconcussive activity levels, symptoms, and neurocognitive performance. J Athl Train. 2008;43:265-274.

103. Aubry M, Cantu R, Dvorak J, et al. Summary and agreement statement of the First International Conference on Concussion in Sport, Vienna 2001. Recommendations for the improvement of safety and health of athletes who may suffer concussive injuries. $\mathrm{Br}$. J. Sports Med. 2002;36:6-10.

104. Lovell MR. The relevance of neuropsychologic testing for sports-related head injuries. Curr. Sports med rep. 2002;1:7-11.

105. Echemendia RJ, Cantu RC. Return to play following sports-related mild traumatic brain injury: the role for neuropsychology. Appl. Neuropsych. 2003;10:48-55.

106. Lovell MR, Collins MW. New developments in the evaluation of sports-related concussion. Curr. Sports med rep. 2002;1:287-292.

107. Grindel SH. The use, abuse, and future of neuropsychologic testing in mild traumatic brain injury. Curr. Sports med rep. 2006;5:9-14.

108. Iverson GL. Outcome from mild traumatic brain injury. Curr Opin Psychiatry. 2005;18:301-317.

109. Watson MR, Fenton GW, McClelland RJ, Lumsden J, Headley M, Rutherford WH. The post-concussional state: neurophysiological aspects. Br. J. Psychiatry. 1995;167:514-521.

110. Lovell M. The management of sports-related concussion: current status and future trends. Clin. Sports Med. 2009;28:95-111.

111. Collie A, Maruff P, McStephen M, Darby DG. Psychometric issues associated with computerised neuropsychological assessment of concussed athletes. Br. J. Sports Med. 2003;37:556-559.

112. McCrea M, Barr WB, Guskiewicz K, et al. Standard regression-based methods for measuring recovery after sport-related concussion. J. Int. Neuropsychol. Soc. 2005;11:5869. 
113. Zakzanis KK, Leach L, Kaplan E. Mild Traumatic Brain Injury. Neuropsychological Differential Diagnosis. Exton: Pennsylvania; 1999:161-171.

114. Binder LM, Rohling ML, Larrabee GJ. A review of mild head trauma: I. Meta-analytic review of neuropsychological studies. J Clin Exp Neuropsychol. 1997;19:421-431.

115. Schretlen DJ, Shapiro AM. A quantitative review of the effects of traumatic brain injury on cognitive functioning. Int Rev Psychiatry. 2003;15:341-349.

116. Collie A, Darby D, Maruff P. Computerised cognitive assessment of athletes with sports related head injury. Br. J. Sports Med. 2001;35:297-302.

117. MCCaffrey RJ, Duff K, Westervelt HJ. Practitioners Guide to Evaluate Change in Neuropsychological Assessment Instruments. New York: Klewer Academic; 2000.

118. Benedict RH, Zgaljardic DJ. Practice effects during repeated administrations of memory tests with and without alternate forms. J Clin Exp Neuropsychol. 1998;20:339-352.

119. Echemendia RJ, Putukian M, Mackin RS, Julian L, Shoss N. Neuropsychological test performance prior to and following sports-related mild traumatic brain injury. Clin $J$ Sport Med. 2001;11:23-31.

120. Mathias JL, Beall JA, Bigler ED. Neuropsychological and information processing deficits following mild traumatic brain injury. J. Int. Neuropsychol. Soc. 2004;10:286-297.

121. Kwok FY, Lee TM, Leung $\mathrm{CH}$, Poon WS. Changes of cognitive functioning following mild traumatic brain injury over a 3-month period. Brain Inj. 2008;22:740-751.

122. Ellemberg D, Leclerc S, Couture S, Daigle C. Prolonged neuropsychological impairments following a first concussion in female university soccer athletes. Clin J Sport Med. 2007; 17:369-374.

123. Erlanger D, Feldman D, Kutner K, et al. Development and validation of a web-based neuropsychological test protocol for sports-related return-to-play decision-making. Arch Clin Neuropsychol. 2003;18:293-316.

124. Erlanger D, Saliba E, Barth J, Almquist J, Webright W, Freeman J. Monitoring Resolution of Postconcussion Symptoms in Athletes: Preliminary Results of a WebBased Neuropsychological Test Protocol. J Athl Train. 2001;36:280-287.

125. Guskiewicz KM, Bruce SL, Cantu RC, et al. Recommendations on management of sportrelated concussion: summary of the National Athletic Trainers' Association position statement. Neurosurgery. 2004;55:891-895.

126. Schatz P, Zillmer EA. Computer-based assessment of sports-related concussion. Appl. Neuropsych. 2003;10:42-47.

127. McCrory P, Makdissi M, Davis G, Collie A. Value of neuropsychological testing after head injuries in football. Br. J. Sports Med. 2005;39.

128. Reddy CC, Collins MW. Sports concussion: management and predictors of outcome. Curr. Sports med rep. 2009;8:10-15.

129. Moser RS, Iverson GL, Echemendia RJ, et al. Neuropsychological evaluation in the diagnosis and management of sports-related concussion. Arch Clin Neuropsychol. 2007;22:909-916.

130. Pellman EJ, Lovell MR, Viano DC, Casson IR. Concussion in professional football: recovery of NFL and high school athletes assessed by computerized neuropsychological testing--Part 12. Neurosurgery. 2006;58:263-274; discussion 263-274.

131. Makdissi M, Collie A, Maruff $P$, et al. Computerised cognitive assessment of concussed Australian Rules footballers. Br. J. Sports Med. 2001;35:354-360. 
132. Warden DL, Bleiberg J, Cameron KL, et al. Persistent prolongation of simple reaction time in sports concussion. Neurology. 2001;57:524-526.

133. CogSport [computer program]. Parkville, Victoria, Australia1999.

134. Bleiberg J, Cernich AN, Cameron K, et al. Duration of cognitive impairment after sports concussion. Neurosurgery. 2004;54:1073-1078; discussion 1078-1080.

135. Erlanger DM, Kaushik T, Broshek D, Freeman J, Feldman D, Festa J. Development and validation of a web-based screening tool for monitoring cognitive status. J. Head Trauma Rehabil. 2002;17:458-476.

136. Iverson GL, Lovell MR, Collins MW. Validity of ImPACT for measuring processing speed following sports-related concussion. J Clin Exp Neuropsychol. 2005;27:683-689.

137. Schatz P, Putz BO. Cross-validation of measures used for computer-based assessment of concussion. Appl. Neuropsych. 2006;13:151-159.

138. Iverson GL, Lovell MR, Collins MW. Interpreting change on ImPACT following sport concussion. Clin. Neuropsych. 2003;17:460-467.

139. McClincy MP, Lovell MR, Pardini J, Collins MW, Spore MK. Recovery from sports concussion in high school and collegiate athletes. Brain Inj. 2006;20:33-39.

140. Collins MW, Field M, Lovell MR, et al. Relationship between postconcussion headache and neuropsychological test performance in high school athletes. Am. J. Sports Med. 2003;31:168-173.

141. Iverson GL, Gaetz M, Lovell MR, Collins MW. Relation between subjective fogginess and neuropsychological testing following concussion. J. Int. Neuropsychol. Soc. 2004;10:904-906.

142. Iverson GL, Brooks BL, Lovell MR, Collins MW. No cumulative effects for one or two previous concussions. Br. J. Sports Med. 2006;40:72-75.

143. Iverson GL, Gaetz M, Lovell MR, Collins MW. Cumulative effects of concussion in amateur athletes. Brain Inj. 2004;18:433-443.

144. Riemann BL, Guskiewicz KM. Effects of Mild Head Injury on P ostural Stability as Measured Through Clinical Balance Testing. J Athl Train. 2000;35:19-25.

145. Geurts AC, Ribbers GM, Knoop JA, van Limbeek J. Identification of static and dynamic postural instability following traumatic brain injury. Arch. Phys. Med. Rehabil. 1996;77:639-644.

146. Guskiewicz KM, Riemann BL, Perrin DH, Nashner LM. Alternative approaches to the assessment of mild head injury in athletes. Med \& Sci in Sports \& Ex's. 1997;29.

147. Peterson CL, Ferrara MS, Mrazik M, Piland S, Elliott R. Evaluation of neuropsychological domain scores and postural stability following cerebral concussion in sports. Clin J Sport Med. 2003;13:230-237.

148. Slobounov S, Tutwiler R, Sebastianelli W, Slobounov E. Alteration of postural responses to visual field motion in mild traumatic brain injury. Neurosurgery. 2006;59:134-139.

149. Catena RD, van Donkelaar P, Halterman CI, Chou LS. Spatial orientation of attention and obstacle avoidance following concussion. Exp Brain Res. 2009;194:67-77.

150. Parker TM, Osternig LR, Lee H, van Donkelaar P, Chou L. The effect of divided attention on gait stability following concussion. Clin. Biomech. 2005;20:389-395.

151. Parker TM, Osternig LR, P VAND, Chou LS. Gait stability following concussion. Med. Sci. Sports Exerc. 2006;38:1032-1040.

152. Parker TM, Osternig LR, van Donkelaar P, Chou LS. Balance control during gait in athletes and non-athletes following concussion. Med Eng Phys. 2008;30:959-967. 
153. Sosnoff JJ, Broglio SP, Ferrara MS. Cognitive and motor function are associated following mild traumatic brain injury. Exp Brain Res. 2008;187:563-571.

154. Notebaert AJ, Guskiewicz KM. Current trends in athletic training practice for concussion assessment and management. J Athl Train. 2005;40:320-325.

155. Moser RS, Schatz P, Jordan BD. Prolonged effects of concussion in high school athletes. Neurosurgery. 2005;57:300-306.

156. Lau B, Lovell MR, Collins MW, Pardini J. Neurocognitive and symptom predictors of recovery in high school athletes. Clin J Sport Med. 2009;19:216-221.

157. Piland SG, Motl RW, Guskiewicz KM, McCrea M, Ferrara MS. Structural validity of a self-report concussion-related symptom scale. Med \& Sci in Sports \& Ex's. 2006;38:2732.

158. Mihalik JP, Stump JE, Collins MW, Lovell MR, Field M, Maroon JC. Posttraumatic migraine characteristics in athletes following sports-related concussion. J. Neurosurg. 2005; 102:850-855.

159. Iverson G. Predicting slow recovery from sport-related concussion: the new simplecomplex distinction. Clin J Sport Med. 2007;17:31-37.

160. King NS, Crawford S, Wenden FJ, Moss NE, Wade DT. The Rivermead Post Concussion Symptoms Questionnaire: a m easure of symptoms commonly experienced after head injury and its reliability. $J$ Neurol. 1995;242:587-592.

161. LaBotz M, Martin MR, Kimura IF, Hetzler RK, Nichols AW. A comparison of a preparticipation evaluation history form and a symptom-based concussion survey in the identification of previous head injury in collegiate athletes. Clin J Sport Med. 2005;15:73-78.

162. Eyres S, Carey A, Gilworth G, Neumann V, Tennant A. Construct validity and reliability of the Rivermead Post-Concussion Symptoms Questionnaire. Clin Rehabil. 2005;19:878887.

163. Chamelian L, Feinstein A, Chamelian L, Feinstein A. Outcome after mild to moderate traumatic brain injury: the role of dizziness. Arch. Phys. Med. Rehabil. 2004;85:16621666.

164. Ghaffar O, McCullagh S, Ouchterlony D, Feinstein A. Randomized treatment trial in mild traumatic brain injury. J. Psychosom. Res. 2006;61:153-160.

165. Heitger MH, Jones RD, Frampton CM, Ardagh MW, Anderson TJ. Recovery in the first year after mild head injury: divergence of symptom status and self-perceived quality of life. J Rehabil Med. 2007;39:612-621.

166. Jakola AS, Muller K, Larsen M, Waterloo K, Romner B, Ingebrigtsen T. Five-year outcome after mild head injury: a prospective controlled study. Acta Neurol Scand. 2007; 115:398-402.

167. Preiss-Farzanegan SJ, Chapman B, Wong TM, Wu J, Bazarian JJ. The relationship between gender and postconcussion symptoms after sport-related mild traumatic brain injury. Pm \& R. 2009;1:245-253.

168. Roe C, Sveen U, Alvsaker K, Bautz-Holter E. Post-concussion symptoms after mild traumatic brain injury: influence of demographic factors and injury severity in a 1-year cohort study. Disabil Rehabil. 2009;31:1235-1243.

169. Stulemeijer M, Vos PE, Bleijenberg G, van der Werf SP. Cognitive complaints after mild traumatic brain injury: things are not always what they seem. J. Psychosom. Res. 2007;63:637-645. 
170. Lannsjo M, af Geijerstam JL, Johansson U, Bring J, Borg J. Prevalence and structure of symptoms at 3 months after mild traumatic brain injury in a national cohort. Brain Inj. 2009;23:213-219.

171. Blinman TA, Houseknecht E, Snyder C, Wiebe DJ, Nance ML. Postconcussive symptoms in hospitalized pediatric patients after mild traumatic brain injury. $J$ Pediatr Surg. 2009;44:1223-1228.

172. Faux S, Sheedy J. A prospective controlled study in the prevalence of posttraumatic headache following mild traumatic brain injury. Pain Med. 2008;9:1001-1011.

173. Register-Mihalik J, Guskiewicz KM, Mann JD, Shields EW. The effects of headache on clinical measures of neurocognitive function. Clin J Sport Med. 2007;17:282-288.

174. Gessel LM, Fields SK, Collins CL, Dick RW, Comstock RD. Concussions among United States high school and collegiate athletes. J Athl Train. 2007;42:495-503.

175. Lew HL, Lin PH, Fuh JL, Wang SJ, Clark DJ, Walker WC. Characteristics and treatment of headache after traumatic brain injury: a focused review. Am J Phys Med Rehabil. 2006;85:619-627.

176. Savola O, Hillbom M. Early predictors of post-concussion symptoms in patients with mild head injury. Eur J Neurol. 2003;10:175-181.

177. Gordon KE, Dooley JM, Wood EP. Is migraine a risk factor for the development of concussion? Br. J. Sports Med. 2006;40:184-185.

178. De Kruijk JR, Leffers P, Menheere PP, Meerhoff S, Rutten J, Twijnstra A. Prediction of post-traumatic complaints after mild traumatic brain injury: early symptoms and biochemical markers. J Neurol Neurosurg Psychiatry. 2002;73:727-732.

179. Register-Mihalik JK, Mihalik JP, Guskiewicz KM. Balance deficits after sports-related concussion in individuals reporting posttraumatic headache. Neurosurgery. 2008;63:7680 .

180. Asplund CA, McKeag DB, Olsen $\mathrm{CH}$. Sport-related concussion: factors associated with prolonged return to play. Clin J Sport Med. 2004;14:339-343.

181. Dischinger PC, Ryb GE, Kufera JA, Auman KM. Early predictors of postconcussive syndrome in a population of trauma patients with mild traumatic brain injury. $J$ Trauma. 2009;66:289-296.

182. Maskell F, Chiarelli $P$, Isles R, Maskell F, Chiarelli P, Isles R. Dizziness after traumatic brain injury: results from an interview study. Brain Inj. 2007;21:741-752.

183. Masson F, Maurette P, Salmi LR, et al. Prevalence of impairments 5 years after a head injury, and their relationship with disabilities and outcome. Brain Inj. 1996;10:487-497.

184. Guskiewicz KM. Balance and mild head injury in athletes. Orthop Phys Ther Clin North Am. 2002;11:143-158.

185. Piland SG, Motl RW, Ferrara MS, Peterson CL. Evidence for the factorial and construct validity of a self-report concussion symptoms scale. J Athl Train. 2003;38:104-112.

186. Control CFD. http://www.cdc.gov/NCIPC/tbi/Facts for Physicians booklet.pdf. Accessed 1/19/2010, 2010.

187. Cantu RC. Posttraumatic Retrograde and Anterograde Amnesia: Pathophysiology and Implications in Grading and Safe Return to Play. J Athl Train. 2001;36:244-248.

188. Iverson GL, Lovell MR, Smith SS. Does brief loss of consciousness affect cognitive functioning after mild head injury? Arch Clin Neuropsychol. 2000;15:643-648.

189. Hinton-Bayre AD, Geffen G. Severity of sports-related concussion and neuropsychological test performance. Neurology. 2002;59:1068-1070. 
190. Lovell M, Collins M, Bradley J. Return to play following sports-related concussion. Clin. Sports Med. 2004;23:421-441.

191. Lau BC, Kontos AP, Collins MW, Mucha A, Lovell MR. Which On-field Signs/Symptoms Predict Protracted Recovery From Sport-Related Concussion Among High School Football Players? Am. J. Sports Med. 2011;39:2311-2318.

192. Kirkwood MW, Yeates KO, Wilson PE. Pediatric sport-related concussion: a review of the clinical management of an oft-neglected population. Pediatrics. 2006;117:1359-1371.

193. Buzzini SR, Guskiewicz KM. Sport-related concussion in the young athlete. Curr. Opin. Pediatr. 2006;18:376-382.

194. Patel DR, Shivdasani V, Baker RJ. Management of sport-related concussion in young athletes. Sports Med. 2005;35:671-684.

195. McDonald JW, Johnston MV. Physiological and pathophysiological roles of excitatory amino acids during central nervous system development. Brain Res Brain Res Rev. 1990;15:41-70.

196. Bruce DA, Alavi A, Bilaniuk L. Diffuse cerebral swelling following head injuries in children: the syndrome of malignant brain edema. J. Neurosurg. 1981;54:170-178.

197. Cantu RC. Second-impact syndrome. Clin. Sports Med. 1998;17:37-44.

198. Covassin T, Swanik CB, Sachs ML. Sex Differences and the Incidence of Concussions Among Collegiate Athletes. J Athl Train. 2003;38:238-244.

199. Fuller CW, Junge A, Dvorak J. A six year prospective study of the incidence and causes of head and neck injuries in international football. Br. J. Sports Med. 2005;39 Suppl 1:i39.

200. Delaney JSM, Lacroix VJM, Leclerc SMD, Johnston KMMDP. Concussions Among University Football and Soccer Players. . Clin J Sport Med. 2002;12:331-338.

201. Barnes BC, Cooper L, Kirkendall DT, McDermott TP, Jordan BD, Garrett WE, Jr. Concussion history in elite male and female soccer players. Am. J. Sports Med. 1998;26:433-438.

202. Boden BP, Kirkendall DT, Garrett WE, Jr. Concussion incidence in elite college soccer players. Am. J. Sports Med. 1998;26:238-241.

203. Barr WB. Neuropsychological testing of high school athletes. Preliminary norms and test-retest indices. Arch Clin Neuropsychol. 2003;18:91-101.

204. Covassin T, Swanik CB, Sachs M, et al. Sex differences in baseline neuropsychological function and concussion symptoms of collegiate athletes. Br. J. Sports Med. 2006;40:923-927.

205. Farace E, Alves WM. Do women fare worse: a metaanalysis of gender differences in traumatic brain injury outcome. J. Neurosurg. 2000;93:539-545.

206. Bazarian JJ, Wong T, Harris M, Leahey N, Mookerjee S, Dombovy M. Epidemiology and predictors of post-concussive syndrome after minor head injury in an emergency population. Brain Inj. 1999;13:173-189.

207. Fenton G, McClelland R, Montgomery A, MacFlynn G, Rutherford W. The postconcussional syndrome: social antecedents and psychological sequelae. Br. J. Psychiatry. 1993;162:493-497.

208. Collins MW, Lovell MR, Iverson GL, Cantu RC, Maroon JC, Field M. Cumulative effects of concussion in high school athletes. Neurosurgery. 2002;51:1175-1179. 
209. Guskiewicz KM, McCrea M, Marshall SW, et al. Cumulative effects associated with recurrent concussion in collegiate football players: the NCAA Concussion Study. JAMA. 2003;290:2549-2555.

210. Schulz MR, Marshall SW, Mueller FO, et al. Incidence and risk factors for concussion in high school athletes, North Carolina, 1996-1999. Am J Epidemiol. 2004;160:937-944.

211. Zemper ED. Two-year prospective study of relative risk of a second cerebral concussion. Am J Phys Med Rehabil. 2003;82:653-659.

212. Gaetz M, Goodman D, Weinberg H. Electrophysiological evidence for the cumulative effects of concussion. Brain Inj. 2000;14:1077-1088.

213. Macciocchi SN, Barth JT, Littlefield L, Cantu RC. Multiple Concussions and Neuropsychological Functioning in Collegiate Football Players. $J$ Athl Train. 2001;36:303-306.

214. Belanger HG, Spiegel E, Vanderploeg RD. Neuropsychological performance following a history of multiple self-reported concussions: a meta-analysis. J. Int. Neuropsychol. Soc. 2010;16:262-267.

215. Van Kampen DA, Lovell MR, Pardini JE, Collins MW, Fu FH. The "value added" of neurocognitive testing after sports-related concussion. Am. J. Sports Med. 2006;34:16301635.

216. Parker TM, Osternig LR, van Donkelaar P, Chou LS. Recovery of cognitive and dynamic motor function following concussion. Br. J. Sports Med. 2007;41:868-873.

217. Keshner EA, Allum JH, Pfaltz CR. Postural coactivation and adaptation in the sway stabilizing responses of normals and patients with bilateral vestibular deficit. Exp Brain Res. 1987;69:77-92.

218. Lacour M, Xerri C. Compensation of postural reactions to free-fall in the vestibular neurectomized monkey. Role of the visual motions cues. Exp Brain Res. 1980;40:103110.

219. Wicke RW, Oman CM. Visual and graviceptive influences on lower leg EMG activity in humans during brief falls. Exp Brain Res. 1982;46:324-330.

220. Peterka RJ. Sensorimotor integration in human postural control. J Neurophysiol. 2002;88:1097-1118.

221. Peterka RJ, Benolken MS. Role of somatosensory and vestibular cues in attenuating visually induced human postural sway. Exp Brain Res. 1995;105:101-110.

222. Bronstein AM, Morland AB, Ruddock KH, Gresty MA. Recovery from bilateral vestibular failure: implications for visual and cervico-ocular function. Acta Otolaryngol Suppl. 1995;520 Pt 2:405-407.

223. Fay BH. Role of the Vestibular System in Postural Control. In: Herdman JS, ed. Vestibular Rehabilitation. Philadelpia: F. A. Davis Company; 2007:32-53.

224. Keshner EA. Postural Abnormalities in Vestibular Disorders. In: Herdman JS, ed. Vestibular Rehabilitation. Philadelphia: F. A. Davis Company; 2007:54-75.

225. Karatas M. Central vertigo and dizziness: epidemiology, differential diagnosis, and common causes. Neurologist. 2008;14:355-364.

226. Krebs DE, Gill-Body KM, Parker SW, Ramirez JV, Wernick-Robinson M. Vestibular rehabilitation: useful but not universally so. Otolaryngol Head Neck Surg. 2003;128:240250 . 
227. Yardley L, Beech S, Zander L, Evans T, Weinman J. A randomized controlled trial of exercise therapy for dizziness and vertigo in primary care. Br. J. Gen. Pract. 1998;48:1136-1140.

228. Horak FB, Jones-Rycewicz C, Black FO, Shumway-Cook A. Effects of vestibular rehabilitation on dizziness and imbalance. Otolaryngol Head Neck Surg. 1992;106:175180.

229. Herdman SJ, Clendaniel RA, Mattox DE, Holliday MJ, Niparko JK. Vestibular adaptation exercises and recovery: acute stage after acoustic neuroma resection. Otolaryngol Head Neck Surg. 1995;113:77-87.

230. Herdman SJ, Schubert MC, Das VE, Tusa RJ. Recovery of dynamic visual acuity in unilateral vestibular hypofunction. Arch Otolaryngol Head Neck Surg. 2003;129:819824.

231. Enticott JC, O'Leary S J, Briggs RJ. Effects of vestibulo-ocular reflex exercises on vestibular compensation after vestibular schwannoma surgery. Otol Neurotol. 2005;26:265-269.

232. Maskell F, Chiarelli P, Isles R, Maskell F, Chiarelli P, Isles R. Dizziness after traumatic brain injury: overview and measurement in the clinical setting. Brain Inj. 2006;20:293305.

233. Griffiths MV. The incidence of auditory and vestibular concussion following minor head injury. J Laryngol Otol. 1979;93:253-265.

234. Herdman JS. Treatment of vestibular disorders in traumatically brain-injured patients. $J$. Head Trauma Rehabil. 1990;5:63-76.

235. Shepard NT, Telian SA, Smith-Wheelock M, Raj A. Vestibular and balance rehabilitation therapy. Ann. Otol. Rhinol. Laryngol. 1993;102:198-205.

236. Gizzi M. Critical review. The efficacy of vestibular rehabilitation for patients with head trauma. J. Head Trauma Rehabil. 1995;10:60-77.

237. Shepard NT, Telian SA, Smith-Wheelock M, Raj A. Vestibular and balance rehabilitation therapy. Ann. Otol. Rhinol. Laryngol. 1993;102:198-205.

238. Ernst A, Basta D, Seidl RO, et al. Management of posttraumatic vertigo. Otolaryngol Head Neck Surg. 2005;132:554-558.

239. Hoffer ME, Gottshall KR, Moore R, et al. Characterizing and treating dizziness after mild head trauma. Otol Neurotol. 2004;25:135-138.

240. Polissar NL, Fay GC, Jaffe KM, et al. Mild pediatric traumatic brain injury: adjusting significance levels for multiple comparisons. Brain Inj. 1994;8:249-263.

241. Hirabayashi S, Iwasaki Y. Developmental perspective of sensory organization on postural control. Brain Dev. 1995;17:111-113.

242. Field M, Collins MW, Lovell MR, Maroon J. Does age play a role in recovery from sports-related concussion? A comparison of high school and collegiate athletes. J. Pediatr. 2003;142:546-553.

243. Vartiainen E, Karjalainen S, Karja J. Vestibular disorders following head injury in children. Int. J. Pediatr. Otorhinolaryngol. 1985;9:135-141.

244. Herdman SJ, Whitney SL. Interventions for the Patient With Vestibular Hypofunction. In: Herdman SJ, ed. Vestibular Rheabilitation. Philadelphia: F.A. Davis Company 2007:309 - 337. 
245. Collins M, Lovell MR, Iverson GL, et al. Examining concussion rates and return to play in high school football players wearing newer helmet technology: a three-year prospective cohort study. Neurosurgery. 2006;58:275-286.

246. Oliaro SM, Guskiewicz KM, Prentice WE. Establishment of Normative Data on Cognitive Tests for Comparison with Athletes Sustaining Mild Head Injury. J Athl Train. 1998;33:36-40.

247. Schmidt JD, Register-Mihalik JK, Mihalik JP, Kerr ZY, Guskiewicz KM. Identifying Impairments after Concussion: Normative Data versus Individualized Baselines. Med Sci Sports Exerc. 2012.

248. Hillier SL, McDonnell M. Vestibular rehabilitation for unilateral peripheral vestibular dysfunction. Clin. Otolaryngol. 2011;36:248-249.

249. Alsalaheen BA, Mucha A, Morris LO, et al. Vestibular rehabilitation for dizziness and balance disorders after concussion. J Neurol Phys Ther. 2010;34:87-93.

250. Whitney SL, Herdman SJ. Physical Therapy Assessment of Vestibular Hypofunction. In: Herdman SJ, ed. Vestibular Rehabilitation. Philadelphia: F.A.Davis; 2007:272 - 308.

251. Wrisley DM, Walker ML, Echternach JL, Strasnick B. Reliability of the dynamic gait index in people with vestibular disorders. Arch. Phys. Med. Rehabil. 2003;84:1528-1533.

252. Whitney SL, Wrisley DM, Marchetti GF, Gee MA, Redfern MS, Furman JM. Clinical measurement of sit-to-stand performance in people with balance disorders: validity of data for the Five-Times-Sit-to-Stand Test. Phys. Ther. 2005;85:1034-1045.

253. Whitney SL, Marchetti GF, Schade A, Wrisley DM. The sensitivity and specificity of the Timed "Up \& Go" and the Dynamic Gait Index for self-reported falls in persons with vestibular disorders. J. Vestib. Res. 2004;14:397-409.

254. Bruininks RH. Bruininks-Oseretsky Test of Motor Proficiency -Owner's Manual. Circle Pines: American Guidance Service; 1978.

255. Powell LE, Myers AM. The Activities-specific Balance Confidence (ABC) Scale. J. Gerontol. A. Biol. Sci. Med. Sci. 1995;50A:M28-34.

256. Shumway-Cook A, woollacott M. Motor Control: Theory and Practical Applications. Baltimore, MD: Williams \& Wilkins; 1995.

257. Wrisley DM, Marchetti GF, Kuharsky DK, Whitney SL. Reliability, internal consistency, and validity of data obtained with the functional gait assessment. Phys. Ther. 2004;84:906-918.

258. Podsiadlo D, Richardson S. The timed "Up \& Go": a test of basic functional mobility for frail elderly persons. J. Am. Geriatr. Soc. 1991;39:142-148.

259. Lord SR, Murray SM, Chapman K, Munro B, Tiedemann A. Sit-to-stand performance depends on sensation, speed, balance, and psychological status in addition to strength in older people. J. Gerontol. A. Biol. Sci. Med. Sci. 2002;57:M539-543.

260. Whitney S, Wrisley D, Furman J. Concurrent validity of the Berg Balance Scale and the Dynamic Gait Index in people with vestibular dysfunction. Physiother. Res. Int. 2003;8:178-186.

261. Shumway-Cook A, Baldwin M, Polissar NL, Gruber W. Predicting the probability for falls in community-dwelling older adults. Phys. Ther. 1997;77:812-819.

262. Hall CD, Schubert MC, Herdman SJ. Prediction of fall risk reduction as measured by dynamic gait index in individuals with unilateral vestibular hypofunction. Otol Neurotol. 2004;25:746-751. 
263. Wrisley DM, Walker ML, Echternach JL, Strasnick B. Reliability of the dynamic gait index in people with vestibular disorders. Arch. Phys. Med. Rehabil. 2003;84:1528-1533.

264. Gill-Body KM, Beninato M, Krebs DE. Relationship among balance impairments, functional performance, and disability in people with peripheral vestibular hypofunction. Phys. Ther. 2000;80:748-758.

265. Whitney SL, Marchetti GF, Morris LO, Sparto PJ. The reliability and validity of the Four Square Step Test for people with balance deficits secondary to a v estibular disorder. Arch. Phys. Med. Rehabil. 2007;88:99-104.

266. Wrisley DM, Whitney SL, Furman JM. Vestibular rehabilitation outcomes in patients with a history of migraine. Otol Neurotol. 2002;23:483-487.

267. Brown KE, Whitney SL, Marchetti GF, Wrisley DM, Furman JM. Physical therapy for central vestibular dysfunction. Arch. Phys. Med. Rehabil. 2006;87:76-81.

268. Viccaro LJ, Perera S, Studenski SA. Is timed up and go better than gait speed in predicting health, function, and falls in older adults? J. Am. Geriatr. Soc. 2011;59:887892.

269. Meretta BM, Whitney SL, Marchetti GF, Sparto PJ, Muirhead RJ. The five times sit to stand test: responsiveness to change and concurrent validity in adults undergoing vestibular rehabilitation. J. Vestib. Res. 2006;16:233-243.

270. Riemann BL, Guskiewicz KM, Shields EW. Relationship between clinical and forceplate measures of postural stability. J Sport Rehabil. 1999:71-82.

271. Bell DR, Guskiewicz K, Clark MA, Padua DA. Systematic Review of the Balance Error Scoring System. Sports Health: A multidisciplinary approach 2011;3:287 -295.

272. Peretz C, Herman T, Hausdorff JM, Giladi N. Assessing fear of falling: Can a sh ort version of the Activities-specific Balance Confidence scale be useful? Mov. Disord. 2006;21:2101-2105.

273. Walker ML, Austin AG, Banke GM, et al. Reference group data for the functional gait assessment. Phys. Ther. 2007;87:1468-1477.

274. Oberg T, Karsznia A, Oberg K. Basic gait parameters: reference data for normal subjects, 10-79 years of age. J. Rehabil. Res. Dev. 1993;30:210-223.

275. Csuka M, McCarty DJ. Simple method for measurement of lower extremity muscle strength. Am. J. Med. 1985;78:77-81.

276. Bressel E, Yonker JC, Kras J, Heath EM. Comparison of static and dynamic balance in female collegiate soccer, basketball, and gymnastics athletes. J Athl Train. 2007;42:4246.

277. McLeod TC, Armstrong T, Miller M, Sauers JL. Balance improvements in female high school basketball players after a 6-week neuromuscular-training program. J Sport Rehabil. 2009;18:465-481.

278. Sabin MJ, Van Boxtel BA, Nohren MW, Broglio SP. Presence of headache does not influence sideline neurostatus or balance in high school football athletes. Clin J Sport Med. 2011;21:411-415.

279. Hunt TN, Ferrara MS, Bornstein RA, Baumgartner TA. The reliability of the modified Balance Error Scoring System. Clin J Sport Med. 2009;19:471-475.

280. Jette AM, Jette DU, Ng J, Plotkin DJ, Bach MA. Are performance-based measures sufficiently reliable for use in multicenter trials? Musculoskeletal Impairment (MSI) Study Group. J. Gerontol. A. Biol. Sci. Med. Sci. 1999;54:M3-6. 
281. Ostchega Y, Harris TB, Hirsch R, Parsons VL, Kington R, Katzoff M. Reliability and prevalence of physical performance examination assessing mobility and balance in older persons in the US: data from the Third National Health and Nutrition Examination Survey. J. Am. Geriatr. Soc. 2000;48:1136-1141.

282. Schaubert KL, Bohannon RW. Reliability and validity of three strength measures obtained from community-dwelling elderly persons. J Strength Cond Res. 2005;19:717720.

283. Schaubert K, Bohannon RW. Reliability of the sit-to-stand test over dispersed sessions. Isokinetics and Exercise Science. 2005;13:119-122.

284. Mong Y, Teo TW, Ng SS. 5-repetition sit-to-stand test in subjects with chronic stroke: reliability and validity. Arch. Phys. Med. Rehabil. 2010;91:407-413.

285. Steffen TM, Hacker TA, Mollinger L. Age- and gender-related test performance in community-dwelling elderly people: Six-Minute Walk Test, Berg Balance Scale, Timed Up \& Go Test, and gait speeds. Phys. Ther. 2002;82:128-137.

286. Rockwood K, Awalt E, Carver D, MacKnight C. Feasibility and measurement properties of the functional reach and the timed up and go tests in the Canadian study of health and aging. J. Gerontol. A. Biol. Sci. Med. Sci. 2000;55:M70-73.

287. Bohannon RW. Comfortable and maximum walking speed of adults aged 20-79 years: reference values and determinants. Age Ageing. 1997;26:15-19.

288. Finnoff JT, Peterson VJ, Hollman JH, Smith J. Intrarater and interrater reliability of the Balance Error Scoring System (BESS). Pm \& R. 2009;1:50-54.

289. Mulsant BH, Kastango KB, Rosen J, Stone RA, Mazumdar S, Pollock BG. Interrater reliability in clinical trials of depressive disorders. Am J Psychiatry. 2002;159:15981600.

290. Reddy CC, Collins MW, Gioia GA. Adolescent sports concussion. . Phys. Med. Rehabil. Clin. N. Am. 2008;19:247-269.

291. Griffiths MV. The incidence of auditory and vestibular concussion following minor head injury. J. Laryngol. Otol. 1979;93:253-265.

292. Kisilevski V, Podoshin L, Ben-David J, et al. Results of otovestibular tests in mild head injuries. Int. Tinnitus J. 2001;7:118-121.

293. Maskell F, Chiarelli P, Isles R. Dizziness after traumatic brain injury: overview and measurement in the clinical setting. Brain Inj. 2006;20:293-305.

294. Geurts AC, Ribbers GM, Knoop JA, van Limbeek J. Identification of static and dynamic postural instability following traumatic brain injury. Arch. Phys. Med. Rehabil. 1996;77:639-644.

295. Shumway-Cook A, Olmscheid R. A systems analysis of postural dyscontrol in traumatically brain-injured patients. J. Head Trauma Rehabil. 1990;5:51-62.

296. Collins M, Lovell MR, Iverson GL, Ide T, Maroon J. Examining concussion rates and return to play in high school football players wearing newer helmet technology: a threeyear prospective cohort study. Neurosurgery. 2006;58:275-286.

297. Hirabayashi S, Iwasaki Y. Developmental perspective of sensory organization on postural control. Brain Dev. 1995;17:111-113.

298. Shepard NT, Telian SA, Smith-Wheelock M. Habituation and balance retraining therapy. A retrospective review. Neurol. Clin. 1990;8:459-475.

299. Jacobson GP, Newman CW. The development of the Dizziness Handicap Inventory. Arch. Otolaryngol. Head Neck Surg. 1990;116:424-427. 
300. Whitney SL, Wrisley DM, Marchetti GF, Furman JM. The effect of age on vestibular rehabilitation outcomes. Laryngoscope. 2002;112:1785-1790.

301. Brown KE, Whitney SL, Marchetti GF, Wrisley DM, Furman JM. Physical therapy for central vestibular dysfunction. Arch. Phys. Med. Rehabil. 2006;87:76-81.

302. Meli A, Zimatore G, Badaracco C, De Angelis E, Tufarelli D. Vestibular rehabilitation and 6-month follow-up using objective and subjective measures. Acta Oto Laryngologica. 2006;126:259-266.

303. Perera S, Mody SH, Woodman RC, Studenski SA. Meaningful change and responsiveness in common physical performance measures in older adults. J. Am. Geriatr. Soc. 2006;54:743-749.

304. Broglio SP, Ferrara MS, Sopiarz K, Kelly MS. Reliable change of the sensory organization test. Clin. J. Sport Med. 2008;18:148-154.

305. Horak FB, Jones-Rycewicz C, Black FO, Shumway-Cook A. Effects of vestibular rehabilitation on dizziness and imbalance. Otolaryngology Head \& Neck Surgery. 1992;106:175-180.

306. Peterson ML, Christou E, Rosengren KS. Children achieve adult-like sensory integration during stance at 12-years-old. Gait Posture. 2006;23:455-463.

307. Guskiewicz KM. Assessment of postural stability following sport-related concussion. Curr. Sports Med. Rep. 2003;2:24-30.

308. Guskiewicz KM, Bruce SL, Cantu RC, et al. National Athletic Trainers' Association Position Statement: Management of Sport-Related Concussion. J Athl Train. 2004;39:280-297.

309. Oliaro S, Anderson S, Hooker D. Management of Cerebral Concussion in Sports: The Athletic Trainer's Perspective. J Athl Train. 2001;36:257-262.

310. Covassin T, Elbin R, 3rd, Stiller-Ostrowski JL. Current sport-related concussion teaching and clinical practices of sports medicine professionals. J Athl Train. 2009;44:400-404.

311. Alla S, Sullivan SJ, Hale L, McCrory P. Self-report scales/checklists for the measurement of concussion symptoms: a systematic review. Br. J. Sports Med. 2009;43 Suppl 1:i3-12.

312. Epley JM. The canalith repositioning procedure: for treatment of benign paroxysmal positional vertigo. Otolaryngol. Head Neck Surg. 1992;107:399-404.

313. Schubert MC, Migliaccio AA, Clendaniel RA, Allak A, Carey JP. Mechanism of dynamic visual acuity recovery with vestibular rehabilitation. Arch. Phys. Med. Rehabil. 2008;89:500-507.

314. Smith-Wheelock M, Shepard NT, Telian SA. Physical therapy program for vestibular rehabilitation. Am J Otol. 1991;12:218-225.

315. Scherer MR, Schubert MC. Traumatic brain injury and vestibular pathology as a comorbidity after blast exposure. Phys. Ther. 2009;89:980-992.

316. Herdman SJ, Schubert MC, Tusa RJ. Strategies for balance rehabilitation: fall risk and treatment. Ann. N. Y. Acad. Sci. 2001;942:394-412.

317. Benjamini Y, Hochberg Y. Controlling the False Discovery Rate: A practical and Powerful Approach to Multiple Testing. Journal of Royal Statistical Society. Series B. 1995;57:289-300. 